\title{
Reliable, Real-Time Acceleration Measurement Toward Automated Monitoring of Driver Behavior
}

\author{
by
}

Michael Rockwood, B. Eng.
A thesis submitted to the Faculty of Graduate and Postdoctoral Affairs in partial fulfillment of the requirements for the degree of

\section{Master of Applied Science in Biomedical Engineering}

\author{
Ottawa-Carleton Institute for Biomedical Engineering \\ Department of Systems and Computer Engineering
}

Carleton University

Ottawa, Ontario, Canada, K1S 5B6

January 2015

C Copyright 2015 Michael Rockwood 


\section{Abstract}

As in other countries, Canada needs to be ready to care for an aging population. Innovation is needed to make health care more efficient and effective. One solution is to monitor health remotely using the Internet. Driving is a high-order function, and its loss can be catastrophic for an elderly person's independence. Unfortunately, physicians do not have the tools they need to adequately determine their patients' driving ability. This thesis details the design and implementation of a remote patient monitoring system that is capable of real-time monitoring, tests its performance, and utilizes it to observe aspects of driver behavior.

Data were collected on sixteen acceleration/deceleration profiles using accelerometers, GPS, and dashboard velocity. The resulting acceleration waveforms were filtered with an adaptive filtering algorithm and compared. Differences between hard and soft acceleration profiles are clearly visible, and notable features of each may be characteristic of individual driver behavior. 


\section{Acknowledgements}

First, I would like to thank my supervisor, Dr. Rafik Goubran, for his guidance and unfailing support throughout my thesis, and for consistently finding time in his incredibly busy schedule to meet with me and keep me focused.

Additionally, I would like to express my sincere gratitude to my office mates, Bruce Wallace and Megan Holtzman, for their technical guidance, and for being patient with me when I would invariably get lost analyzing data or filling out forms. I would also like to thank Vilas Joshi, Stephanie Bennett, Zhaofen Ren, and all my other friends in our corner of the Minto Building for their mental and emotional support during this last, grueling year spent hammering out my thesis.

Thanks must also go out to my girlfriend, Alejandra, who has been by my side every single day of this degree. She has been incredibly patient and supportive during all the late nights I spent working and cursing at my computer monitor, and my endless stressing over meetings and deadlines throughout my degree.

Finally, I would like to thank my parents and my brother, for their constant, unconditional love and support throughout my entire life, and in these last two years for spending hours with me on the phone or on Skype discussing and editing my thesis from a thousand kilometres away. I couldn't have made it this far without you. 


\section{Table of Contents}

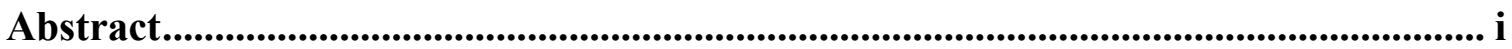

Acknowledgements .................................................................................................................. ii

Table of Contents ............................................................................................................................. iii

List of Tables ........................................................................................................................ vi

List of Figures............................................................................................................................. vii

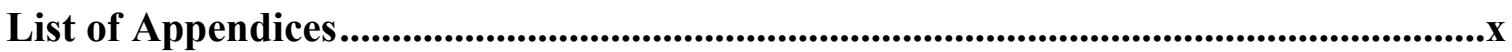

List of Acronyms ........................................................................................................................... xi

List of Symbols ............................................................................................................................ xii

Chapter 1: Introduction .......................................................................................................1

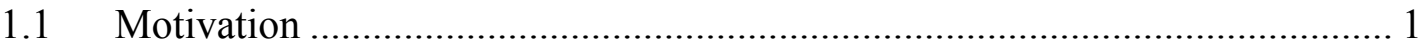

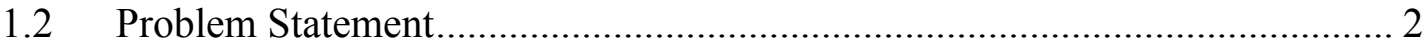

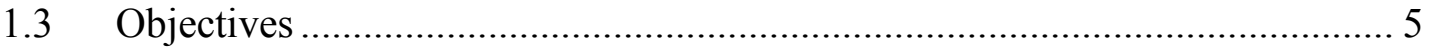

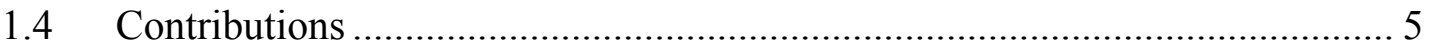

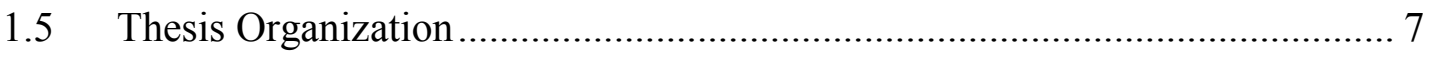

Chapter 2: $\quad$ Background Review .........................................................................................9

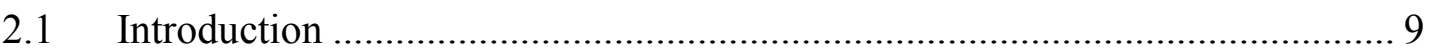

2.2 Challenges of an Older Population ............................................................. 9

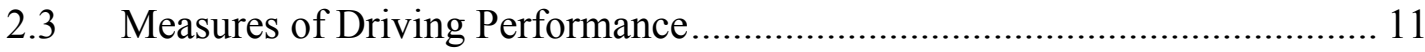

2.4 Accelerometer Calibration....................................................................... 12

$2.5 \quad$ Remote Patient Monitoring ………………………..................................... 15

2.6 Real Time Operating System................................................................... 17

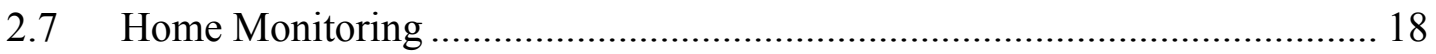




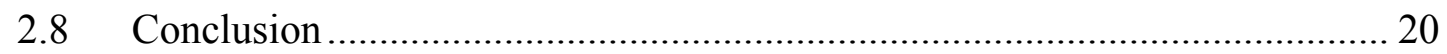

Chapter 3: $\quad$ Equipment Setup and Experimentation............................................21

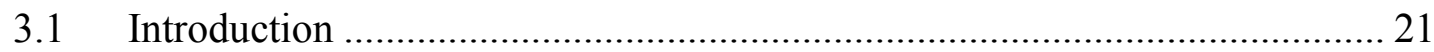

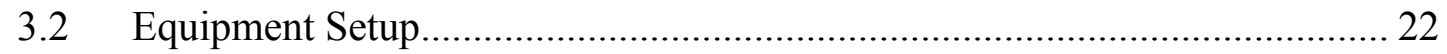

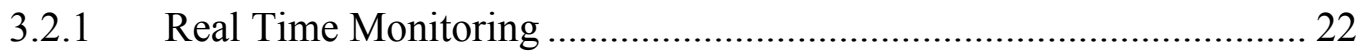

3.2.2 Accelerometer Calibration............................................................... 23

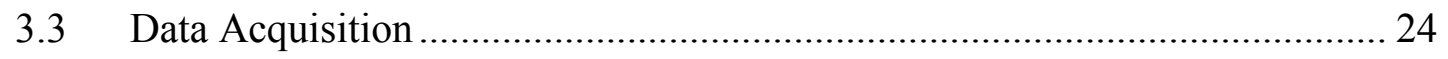

3.3.1 Real Time Data Processing.............................................................. 24

3.3.2 Driving Data ................................................................................. 26

Chapter 4: Remote Patient Monitoring System Design and Implementation .....28

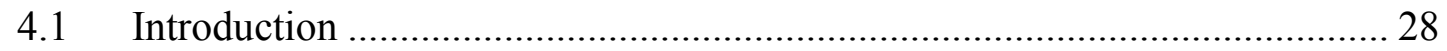

$4.2 \quad$ System Design ......................................................................................... 29

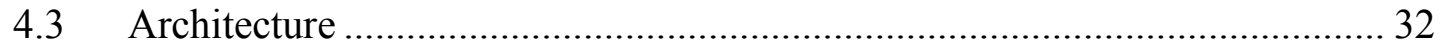

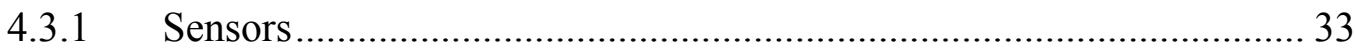

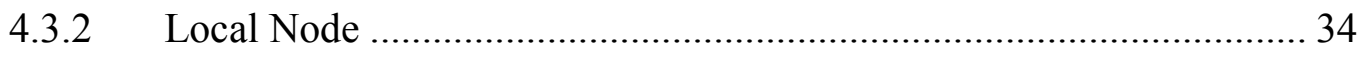

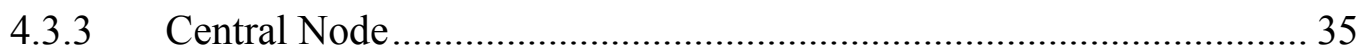

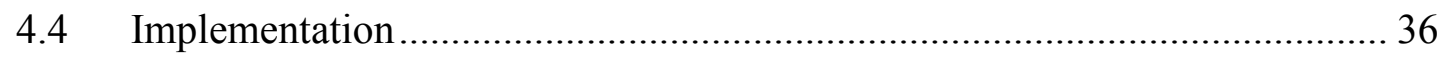

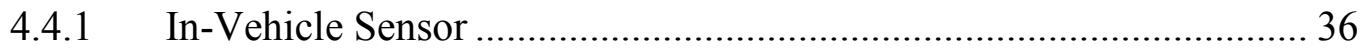

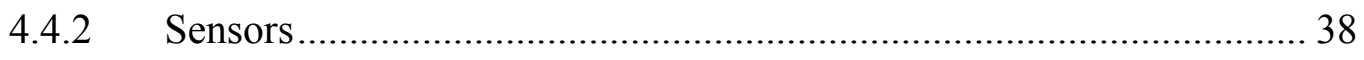

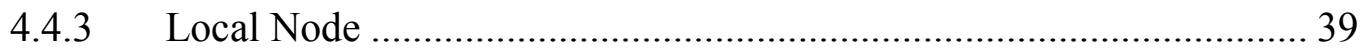

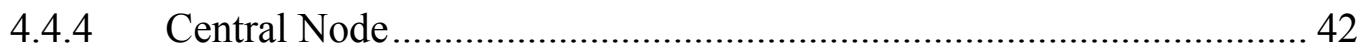

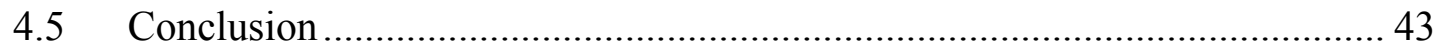

Chapter 5: $\quad$ Real-Time Operating System ........................................................................44

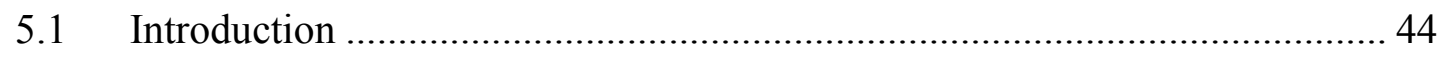

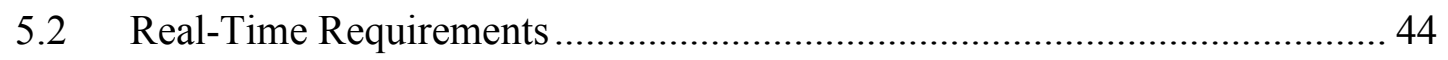




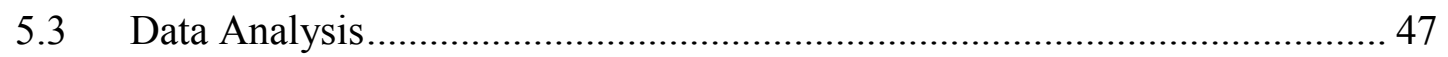

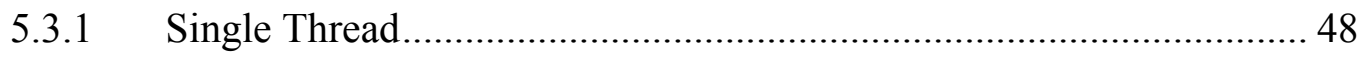

5.3.2 Five Sensors of the Same Priority ......................................................... 49

5.3.3 Four Low Priority Sensors and One High Priority Sensor ................... 50

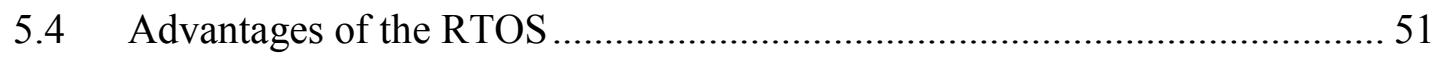

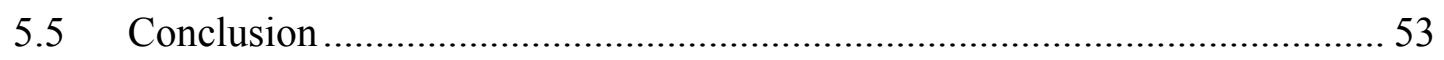

Chapter 6: Adaptive Accelerometer Calibration............................................................54

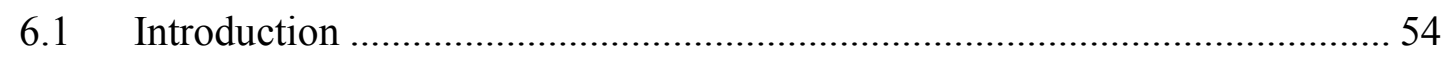

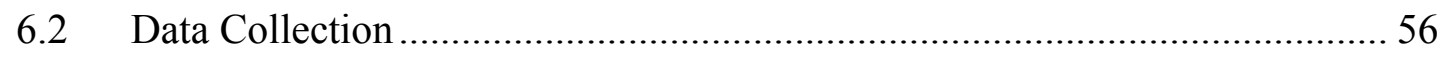

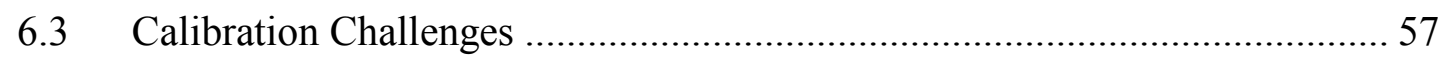

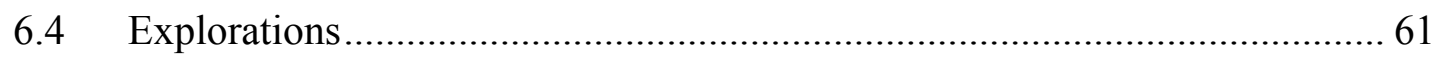

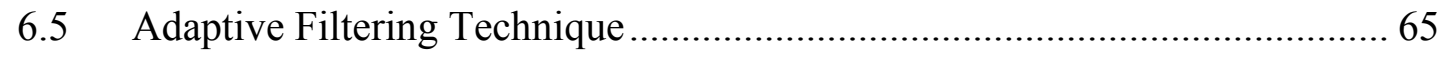

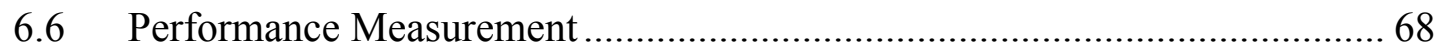

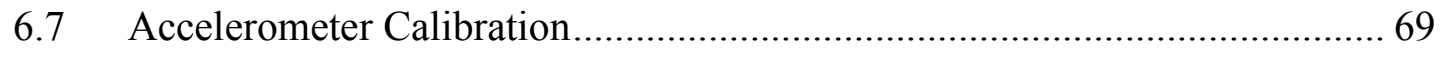

6.7.1 Error Signal Power ........................................................................... 75

6.7.2 Corrected Accelerometer Correlation.................................................. 76

6.8 Implications of the Adaptive Filtering Technique........................................... 78

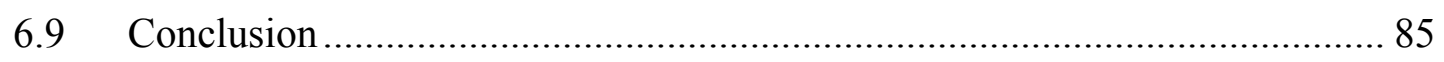

Chapter 7: Conclusions ....................................................................................................86

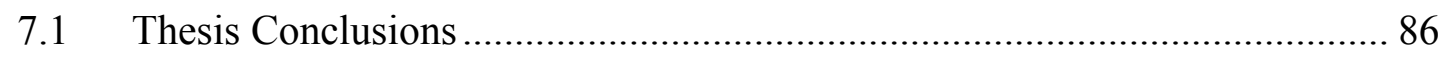

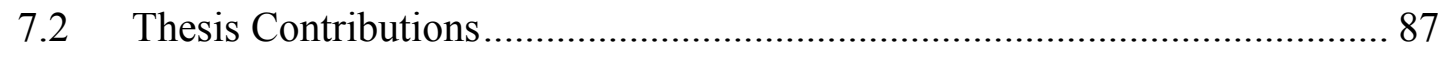

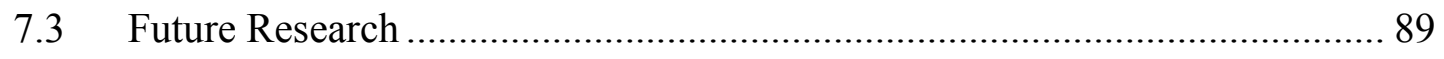

References ..................................................................................................................................91

Appendix A: Sequence Diagram of the RPM System.....................................................102 


\section{List of Tables}

Table 1: Data collected by the Candrive Persentech OttoView-CD sensor.

Table 2: Sampling and data rates for various types of common biomedical measurement

devices

Table 3: Initial attempts to calibrate the data using a variety of methods. These were

largely probing in nature, as an attempt to get an idea of the shape of the data.

Table 4: A summary of the average total energy of the error in each acceleration/deceleration profile, measured using step and ramp deltas for both the GPS and OBDII reference voltages.

Table 5: Summary of the uncorrected acceleration sensor correlation data for each of the smart phone acceleration profiles.

Table 6: Summary of the corrected acceleration sensor correlation data for each of the smart phone acceleration profiles using the GPS velocity reference and step delta function. 


\section{List of Figures}

Figure 1: Real time monitoring equipment setup. …….............................................. 22

Figure 2: Real time monitoring experiment equipment setup. ..................................... 25

Figure 3: Accelerometer stack with top sides (positive $\mathrm{Y}$ axis) oriented toward the front of the vehicle

Figure 4: Accelerometer stack with left sides (positive $\mathrm{X}$ axis) oriented toward the front of the vehicle. 27

Figure 5: Local RPM system design ......................................................................... 29

Figure 6: Centralized RPM system design.............................................................. 30

Figure 7: Centralized local RPM system ……………….......................................... 32

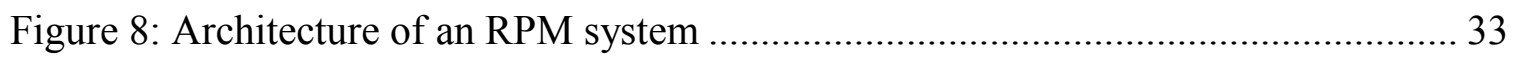

Figure 9: Candrive Persentech OttoView-CD sensor [59] ............................................ 37

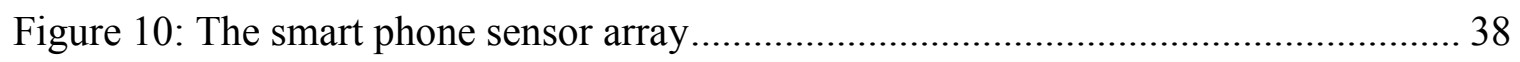

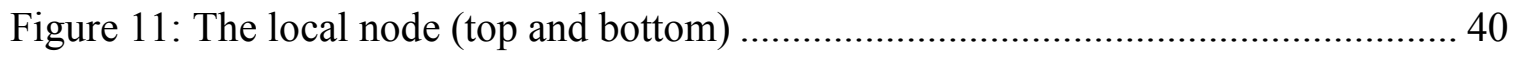

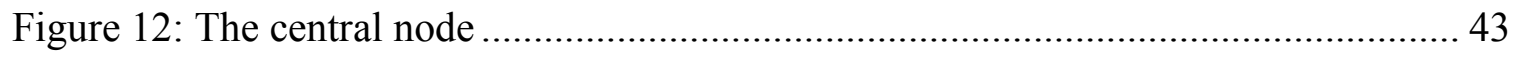

Figure 13: Real-time processing threshold ................................................................... 46

Figure 14: Completion time for data from a single sensor .............................................. 48

Figure 15: Completion time for data from five sensors being processed at equal priority49

Figure 16: Completion time for data from one high priority sensor and four low priority

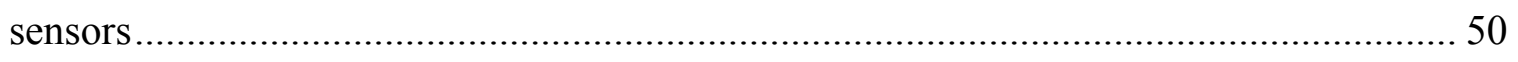

Figure 17: Illustration of different acceleration patterns ............................................... 55

Figure 18: Effect of vibration noise on the accelerometer waveform ............................. 57 
Figure 19: Effects of baseline drift on the acceleration waveform 58

Figure 20: Illustration of accelerometer offset error .............................................. 59

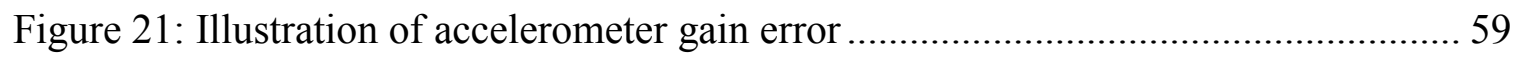

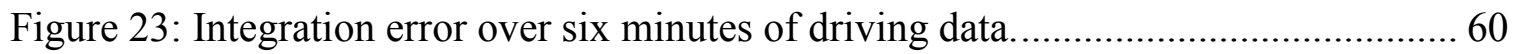

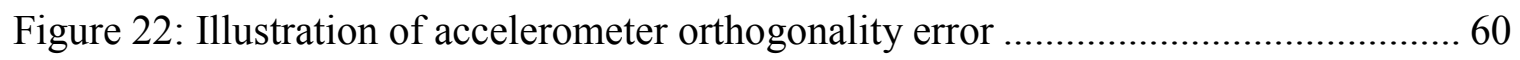

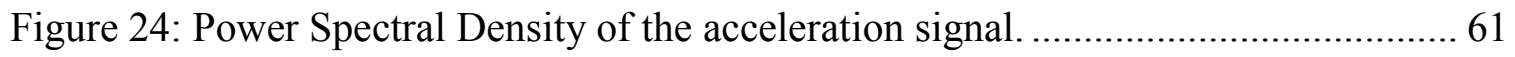

Figure 25: Effect of a high pass filter on the acceleration signal................................. 63

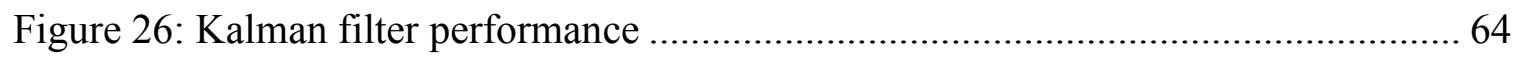

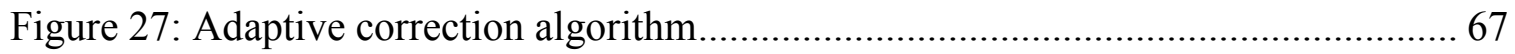

Figure 28: Example acceleration/deceleration profile in time and frequency domain. .... 69

Figure 29: Accelerometer calculated velocity before and after calibration .................... 70

Figure 30: Acceleration profile using a ramp-shaped delta function compared to GPS

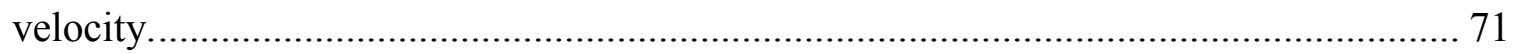

Figure 31: Acceleration profile using a ramp-shaped delta function compared to OBDII

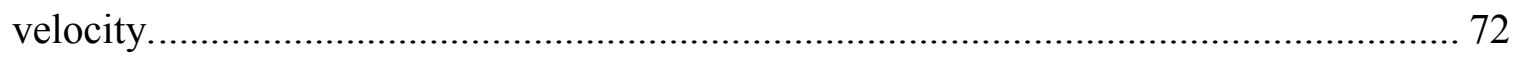

Figure 32: Acceleration profile using a step-shaped delta function compared to GPS

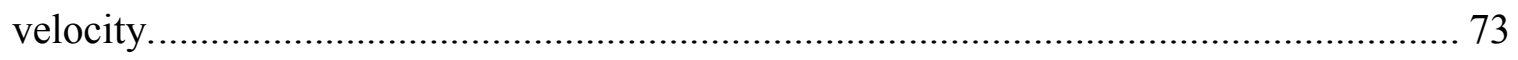

Figure 33: Acceleration profile using a step-shaped delta function compared to OBDII

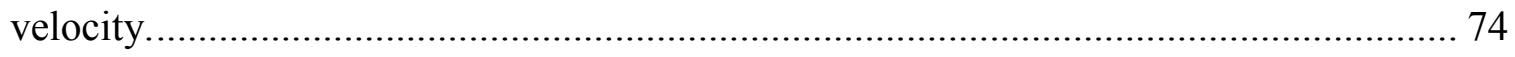

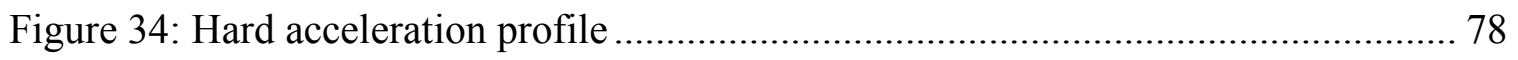

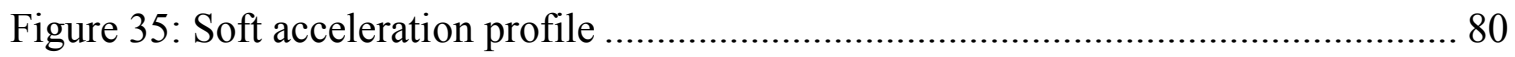

Figure 36: comparison of mean hard acceleration vs soft acceleration ........................ 81

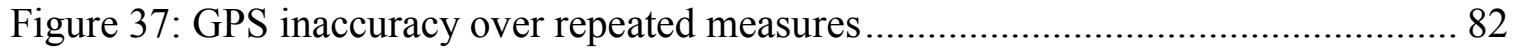


Figure 38: Illustration showing relative GPS error vs distance between measurements .. 84

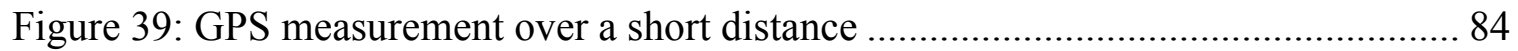

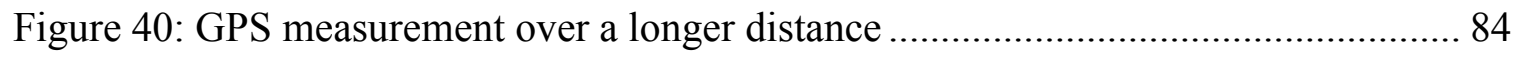




\section{List of Appendices}

Appendix A: Sequence Diagram of the RPM System ........................................................98 


\section{List of Acronyms}

$\begin{array}{ll}\text { RPM } & \text { Remote Patient Monitoring } \\ \text { MMSE } & \text { Mini-Mental State Examination } \\ \text { ADL } & \text { Activity of Daily Living } \\ \text { IMU } & \text { Inertial Measurement Unit } \\ \text { GPS } & \text { Global Positioning System } \\ \text { OS } & \text { Operating System } \\ \text { GPOS } & \text { General-Purpose Operating System } \\ \text { RTOS } & \text { Real Time Operating System } \\ \text { BAN } & \text { Body Area Network } \\ \text { ECG } & \text { Electrocardiogram } \\ \text { EEG } & \text { Electroencephalogram } \\ \text { EMG } & \text { Electromyogram } \\ \text { HABAM } & \text { Hierarchical Assessment of Balance and Mobility } \\ \text { OBDII } & \text { On-Board Diagnostic } \\ \text { RFID } & \text { Radio Frequency Identification }\end{array}$




\section{List of Symbols}

$\begin{array}{ll}d_{R T} & \text { Real-time processing threshold } \\ l_{W} & \text { Sample window length } \\ r_{D} & \text { Incoming data rate } \\ l a g_{R T} & \text { Execution time lag } \\ t_{E} & \text { Execution time } \\ l a g_{t o t a l} & \text { Total execution time lag } \\ d_{W} & \text { Duration of system load } \\ l a g_{\%} & \text { Fractional execution time lag } \\ f_{S P} & \text { Smart phone sampling rate } \\ f_{C D} & \text { Candrive sensor sampling rate } \\ A_{m} & \text { Measured acceleration } \\ A_{a} & \text { Actual Acceleration } \\ d & \text { Accelerometer drift } \\ V_{a} & \text { Actual velocity } \\ Z_{v} & \text { Velocity noise } \\ Z_{I} & \text { Integration noise } \\ R & \text { Decimation ratio } \\ V_{p} & \text { Measured velocity } \\ V_{m} & \end{array}$


$A_{c}$

$\epsilon_{n}$

$\Delta_{\text {step }}$

$\Delta_{\text {ramp }}$
Corrected acceleration

Velocity error

Step shaped delta

Ramp shaped delta 


\section{Chapter 1:}

\section{Introduction}

\subsection{Motivation}

As the population ages, so too does the prevalence of frailty and chronic illness. Some studies have suggested that, in Canada, by 2026 the number of people over the age of 65 will have increased by more than 50\% [1][2]. Older adults already utilize a large proportion of healthcare resources [1]. In many parts of the world, traditional health care providers such as hospitals, nursing homes, and assisted living facilities are operating nearly at capacity. It is clear that with the way the current system operates, it is not going to be able to keep up with the increase in demand in the long term. One proposed method for reducing the strain on the healthcare system is to keep frail older adults with chronic health problems living independently for longer, leveraging the ubiquity of the Internet to monitor their health status remotely. Even so, monitoring technology and applications will need to better adapt to the needs of older adults.

Two major factors are recognized as key contributors to the premature institutionalization of older adults: the tremendous loss of independence caused by losing their driver's license [3], and the fear of risk-averse offspring who live some distance from them [4]. The latter issue has been tackled by many research groups over a number 
of years, but the former has received notably less attention, despite having been identified for a long time [5].

\subsection{Problem Statement}

One key factor to maintaining the independence of older adults is being able to effectively assess their driving. The challenge posed by a large number of elderly people driving, some of whom might knowingly or unknowingly be at a substantially increased risk to themselves and others, is difficult. It is clear that not taking away the license of an elderly person at the appropriate time can lead to dangerous situations for everyone on the road and tragedy for some. No such incident stands out more in Canada than the horrific story of 45 year old corporate executive, and mother of two, Elizabeth Kidnie, who in 2000 was struck in a crosswalk by then 84 -year old Pilar Hicks and dragged along by the undercarriage of the car for more than a kilometer. Even after striking her directly with the front of her car, Mrs. Hicks continued to drive home, somehow failing to notice either that she had hit anyone or that she was dragging anything, even though the body only became dislodged as Mrs. Hicks pulled into her driveway. Investigators were easily able to trace the path that Mrs. Hicks had followed by the trail left by her unfortunate victim's remains [6].

Horrific loss of life is, however, only one part of the story. The loss of a driver's license greatly impacts the independence and quality of life of an older person, and on their family members. This includes care providers who will have to add driving chores to their current list of duties, something that, actually, also increases their own risk. Loss of transportation reduces access to basic needs, such as the grocery store and the 
pharmacy, and makes older adults dependent on others to travel to and from appointments and meetings [3]. Importantly, too, losing access to a car makes social interaction considerably more difficult, and can lead to issues of social abandonment, which has been shown to be correlated with a significant increase in rates of depression, worsening of dementia, and even mortality [3][7]. Ultimately, losing access to the car can even lead to institutionalization for the elderly person, including some of those who are still otherwise capable of looking after themselves.

At present, physicians have only a limited set of tests for making decisions about whether an individual's driver's license should be taken away. These tests are typically performed in the office, rather than on the road, so that their generalizability is not certain, even if their internal validity can be demonstrated [8]. This means that many older people could well have their drivers' licenses revoked unfairly, whereas others may be allowed to drive well beyond when they should be able to [9]. It is clear that some better measure of real-world driving ability is needed.

Another common risk factor for institutionalization is the concerns of risk-averse offspring. Many adult children of older adults eventually become concerned for the stability of their parent's health and for their ability to manage their own well-being, especially if the parent is suffering from any sort of cognitive decline. Despite these concerns, many older adults may be fully capable of living independently, or with minimal support in the form of medication prompting, heavy lifting, or other maintenance chores. Installing some kind of RPM system to monitor health and provide reminders or check that medications were being taken regularly would help alleviate the concerns of 
the children, while at the same time improving the independence and quality of life of the older person.

In addition to the potential benefits offered by an in-home RPM system, an accurate measure of real-world driving ability cannot be indifferent to everyday performance in other activities that go with good driving. Indeed, information from how those activities are performed can be leveraged to give a comprehensive understanding of driving performance. Consequently, it is not necessary to always wait for recording of their driving errors to understand when older people might be at risk. It has been shown that everyday function [10][11][12], physical activity [13], and executive function [12][14] are each key to driving performance. Commonly, however, many of these functions are measured only by neurocognitive tests that have an uncertain relationship with real-world performance, real-world functional measures, and measures of mobility and balance [15][16][17][18]. Additionally, although many elderly people can be frail, frailty is under-recognized as a factor in driving impairment. For example, an October 2014 PubMed search with terms "driving assessment" and "dementia" resulted in 179 entries, while "driving assessment" and "function" returned 219, and "driving assessment" and "frailty" returned only 6 entries. This is the case even though frailty is consistently linked to problems with mobility and balance [19], and function [20], and is more closely linked to a range of adverse outcomes than are other factors associated with driving problems, such as orthostatic hypotension [21][22]. This suggests, then, that there is also a place for continuous remote monitoring in the home in providing a full context for assessing driving ability. 


\subsection{Objectives}

The first objective of this thesis is to design a Remote Patient Monitoring (RPM) system that is capable of monitoring aspects of behavior or health status in real time. Such a system should be adaptable for use in the home for ambient in-home monitoring or in the car for mobile monitoring, thereby giving a more complete profile of an individual's health and enhancing the understanding of their driving performance and potential. The second objective is to make use of the RPM system to begin the process of fairly assessing driver competence by demonstrating a tool that could be used in the development of a 'driver signature', a unique identifier based on driving habits.

\subsection{Contributions}

The following is a list of research contributions that were made during the development of this thesis; each contribution will be explained in more detail in the following chapters:

1. The first contribution was the design and development of a full framework for a remote monitoring system. This system is compatible with the on-board operating system of many types of vehicles, with several types of powerful microcontrollers, and with Blackberry smartphones, making it an effective system for 24-hour automatic monitoring in any environment.

a. M. Rockwood, K. Sullivan, R. Goubran, "Remote Patient Monitoring Using the QNX Real Time Operating System," presented at The $3^{\text {rd }}$ 
annual conference of Healthcare Support through Information Technology Enhancements (hSITE), Montreal, QC, 2013.

b. M. Rockwood, V. Joshi, K. Sullivan, R. Goubran, "Using a Real-Time Operating System for Multitasking in Remote Patient Monitoring," in proc. 2014 IEEE International Symposium on Medical Measurements and Applications (MeMeA), pp. 475-479, 2014.

2. The second contribution was the development of an experiment and data acquisition application to collect acceleration data from a vehicle outfitted with Candrive sensors. The goal was to use the data to assist in the development of a 'driver signature' that could be used to track driver performance and ultimately help inform decisions about driving cessation among elderly people.

a. B. Wallace, M. Rockwood, R. Goubran, F. Knoefel, S. Marshall, M. Porter. "Measurement of Vehicle Acceleration in Studies of Older Drivers from GPS Position and OBDII Velocity Sensors," submitted to 2015 IEEE International Symposium on Medical Measurements and Applications (MeMeA).

3. The third contribution was to design and implement an adaptive filtering algorithm to calibrate the accelerometer data using two measures of the car's velocity as reference values. This allows the full use of the higher sampling rate and lower power accelerometer without having to manage the complex drift and integration error issues normally associated with accelerometer measurements. This contribution likely has a more widespread potential for use in other settings where GPS sampling is not sufficiently energy efficient for longer term use. 
a. M. Rockwood, B. Wallace, R. Goubran, F. Knoefel, S. Marshall. "Adaptive Drift Calibration of Accelerometers with Direct Velocity Measurements," accepted at 2015 IEEE International Instrumentation and Measurement Technology Conference (I2MTC).

\subsection{Thesis Organization}

- Chapter 2 will provide a literature review about the unique medical challenges posed by elderly people, about measures of driving performance, and about accelerometer calibration. It also provides background information on remote patient monitoring systems in the car and at home, and on real-time operating systems.

- Chapter 3 explains the specific hardware layout of each test. Further, it discusses the experiment setup and data acquisition.

- Chapter 4 presents a detailed look at the design of the RPM system. It also describes its implementation for testing purposes.

- Chapter 5 details the experiment with the use of a real time operating system in a remote patient monitoring system. It describes the importance of the real-time processing threshold, and shows what how a real time operating system can outperform a general purpose one, and how using a dedicated local node is preferable to using a general-use device such as a PC or smart phone. 
- Chapter 6 presents the adaptive filtering technique used for accelerometer calibration. It shows how this method can be used to prevent drift and eliminate many of the integration problems associated with accelerometers, allowing them to be used to more accurately describe movement and track position and velocity.

- Chapter 7 summarizes the body of the thesis, enumerates the contributions made and contains a list of recommendations for future work. 


\section{Chapter 2:}

\section{Background Review}

\subsection{Introduction}

This chapter describes the challenges to the healthcare system that arise from trying to manage the needs of an ageing population. It then outlines how RPM systems show promise in helping to address some of these challenges. Further, the chapter illustrates the differences between categories of RPM systems. In addition, this chapter defines the real time operating system, and explains its importance in remote monitoring. It then provides a review of existing RPM systems for older adults, including both those that are installed in the home and those that are worn on the body.

\subsection{Challenges of an Older Population}

The field of remote health monitoring is expanding rapidly [23]. Monitoring devices have been developed for use in everything from daily management of chronic conditions such as diabetes [24] to keeping track of vital signs of climbers on Mount Everest [25]. Despite these successes in other fields of health management, when 
employed in monitoring elderly people, RPM systems have had limited impact. In particular, RPM systems have not been shown to reduce the incidence of acute illness, even if they have been shown to better identify some negative health trends [26]. Likewise, results have been mixed in relation to their cost effectiveness; this is the case even though early interventions, such as might be expected from the early detection allowed by RPM systems, are typically less expensive than hospital admissions [27][28].

Despite these more discouraging results, it must be recognized that to now, comparatively few systems have been designed for use in maintaining the independence of elderly people and these systems have tended to be under-developed [5]. For example, to date the monitoring systems employed with older adults have tended to be expensive to install, and are often either narrow in scope and used primarily for research into specific disorders, or are designed only to detect acute illness, such as falls [29][30]. In addition, only a few existing systems take advantage of continuous monitoring technology, and many instead rely on daily input from the patient and oblige someone to review the data [31]. In short, even in this limited scope of RPM systems specifically for older adults, more technical development, and more research, is needed if the potential of such systems in reducing hospital admissions and increasing quality of life is to be fully realized.

Perhaps the most significant reasons for the relatively little success in creating RPM systems to maintain the independence of elderly people are the unique challenges in diagnosis and treatment that come with aging, and that these challenges have not readily been accommodated by RPM systems in use to date. As summarized in a recent Nature commentary, the central challenge to be addressed is that "[t]he problems of old age 
come as a package" [32]. Older people commonly have multiple chronic disorders [20], and acute illness in them often presents in ways which are inconsistent, difficult to predict, and are less clearly related to what they have wrong - e.g. heart attacks may not present with chest pain or shortness of breath, but with simple tiredness, or even confusion [33]. In failing so far to successfully handle the challenge presented by managing multiple health problems, RPM systems are not alone. Indeed, the Nature commentary goes on to say that, despite having some understanding of the challenge of the complexity of needs and illness in ageing " [t] hese insights have made hardly a dent in human medicine" [32]. Even so, this is not where the challenges end. Older people are often less likely to be accepting of technology, unless it has been proven to reduce the risk of having to leave their own homes and be institutionalized [34][35][36]. These challenges make many existing RPM technologies unsuitable for use in their health management.

\subsection{Measures of Driving Performance}

Drivers who have been diagnosed with dementia are at an increased risk of a number of driving problems such as: getting lost, driving too slowly, making dangerous left hand turns, forgetting to wear their seatbelt, scraping their vehicles while navigating narrow passageways, and being involved in a collision [37][38][39]. Furthermore, without access to up-to-date driving tests, physicians do not have the tools to definitively say whether some patients are fit to drive [40]. It is clear that those with moderate or severe dementia are not capable of operating any sort of vehicle, but in those with an early diagnosis of dementia it is much less clear. A patient's score on the Mini-Mental 
State Examination (MMSE) is often used as a guideline, and some have reported that specific tests of cognitive function, such as visuospatial or selective attention tasks can also predict driving performance [41][42]. However, no consensus has been reached, and still others have reported that cognitive testing alone, including tests that are often used exactly for this purpose, are not sufficient to predict driving ability [43][44][45]. Several studies have shown that physical function and the ability to independently perform activities of daily living (ADLs; includes activities such as bathing, dressing, toileting, grooming, eating, and instrumental ADLs, such as housekeeping, using the telephone, shopping, managing money and taking medications) must also be examined in order to get an accurate picture of driving ability. In short, driving ability should not be considered in isolation. Rather, it can be thought of as a high-order function, in that it integrates a large number of capabilities - both cognitive and non-cognitive. Since the human body can be thought of as a complex system, and when complex systems fail, they typically fail in their highest order functions first, tracking driving ability can be a sensitive sign of system performance overall. Especially in that light, driving performance should be considered in the context of overall performance in everyday function.

\subsection{Accelerometer Calibration}

The advent of the smart phone has created a world in which most people now carry a substantial amount of processing power on their person at all times. In addition, all modern smart phones come equipped with a wide array of sensing devices: a microphone, a three-axis accelerometer, a gyroscope, and a magnetometer. They also 
provide a link to the Global Positioning System satellite network, allowing tracking of movement outside the home to an accuracy of a few meters. This powerful combination of capabilities has many possible applications in medicine.

Inertial Measurement Units (IMUs) are devices that typically consist of an accelerometer, a gyroscope, and (usually) a magnetometer to measure movement in various dimensions. They have been in use for decades, but only comparatively recently manufacturing methods have made them small and inexpensive enough to be installed in many types of consumer electronics, most notably in smart phones. Accelerometers measure linear acceleration in three dimensions, while gyroscopes measure rotational movement, and magnetometers measure orientation with respect to magnetic north. Of these three components of the IMU, accelerometers in particular have become extremely popular. They are less expensive, lower power consumption, and offer much higher sensitivity than many other methods of measuring movement, such as the Global Positioning System (GPS). Additionally, they are widely available as they are a standard sensor included in all smart phones, which have been nearly ubiquitously adopted by the general public. They have been widely employed by many research groups for an extensive variety of purposes, from detection of movement during sleep to robot control systems. One common application of accelerometers is to characterize human movement [46][47]. For example, they are currently being used to evaluate the efficacy of joint replacement surgery [48] and they are useful in gait analysis which may help quantify drug treatment effects in patients with movement disorders such as Parkinson's disease [49] Recently, Calore et al. have proposed an application of accelerometers for on board 
vehicle image correction and have demonstrated that calibration of the accelerometer is a key factor in its performance [50].

Techniques for accelerometer calibration have been reported by several research groups. One group proposes an auto calibration technique for MEMS accelerometers using the gravity vector while the accelerometer is motionless [51]. Another proposes an external system for the calibration of an accelerometer [52]. Still another proposes an apparatus that can provide known accelerations for the laboratory calibration of accelerometers [53]. Others have proposed algorithms that use robotic arms or similar devices to ensure precise accelerometer orientation during calibration [54][55]. Although these methods may in fact improve accelerometer performance, they still present difficulties in that each requires the use of specialized external equipment, and none provide any assistance while the accelerometer is in use.

Given these difficulties, most accelerometer-based measurement systems do not include position tracking. Instead, most focus on tracking an overall 'activity level' [56][57] or on characterizing specific sets of movements, such as sitting, standing, lying down, or walking [58]. These systems have been studied by a number of researchers in recent years, and many have achieved very high measurement accuracy in the lab. Gyllensten et al, however, have shown that these accuracies can be misleading, as movements in the real world are rarely as clearly defined as they are in the laboratory setting [58].

The application of accelerometers to the study of driving performance, particularly for assessing the abilities of older drivers, is potentially a promising area of research. A number of research groups have proposed in-vehicle sensor systems that 
allow the recording of driving behaviors [59][60][61][62][63][64]. These studies have shown that sensor equipment is capable of providing a clearer understanding of drivers' performance, but thus far, none of these studies have made use of accelerometer sensors with large user populations because of the challenges with the deployment and use of these sensors.

\subsection{Remote Patient Monitoring}

Broadly speaking, RPM systems fall into two categories. The first, which has become increasingly common since the widespread adoption of the smartphone and large 2, 3, and 4G network coverage, is the mobile RPM system. These systems can be installed in a vehicle, or even worn directly on the body. They can monitor selected aspects of the environment, but they can also directly monitor the activities of the subject/patient (such as through the use of GPS or an accelerometer) or aspects of their physiology (such as heart rate, breathing, and even mobile ECG or EEG monitoring). These systems either store the output locally or transmit it to a central server through a smart phone connected to a cellular network.

The second category is the environmental or ambient RPM system. These systems are installed in a home or hospital environment, and typically include motion tracking systems, door sensors, thermostats, light sensors, or pressure sensors. These systems are not generally capable of directly measuring physiological signals, but can often be used to monitor ADLs such as bathroom and kitchen use, medication scheduling, and mobility.

Most RPM systems have been developed to track single illnesses or individual chronic conditions. For instance, there are research groups working on improving blood 
glucometers for diabetics [65][66], recording lung function [67], observing pacemaker function [68], detecting atrial fibrillation [69], and monitoring body temperature, blood pressure, and heart rate [70][71][72]. These devices are suitable for use in younger people, who may in fact have only a single chronic disorder. On the other hand, elderly people, who often have many health problems, would need to wear or install several single-system monitoring devices and make use of them all simultaneously, which would not be serving their needs adequately. For this reason, systems that monitor many body systems simultaneously - which can be achieved through monitoring high-order functions such as attention, mobility, balance, and function - may be better suited to the needs of older adults. Such high order functions are less disease specific: for instance, mobility impairment can reflect problems in heart function, lung function, or problems in nerves, muscles, bones or joints. Ambient sensor systems can provide a great deal of data for measuring these high order functions. Unfortunately, comparatively few remote monitoring systems are designed with these high order functions in mind, and instead focus on specific aspects of disease such as fall detection [73] and wandering [74].

Despite these issues from what is, after all, the early days of RPM system development, these approaches have the potential to provide improved quality of care as well as to reduce overall healthcare costs [75][35]. They are already often utilized to monitor chronic conditions, but they can also be employed to detect acute medical events and emergency situations simultaneously. Monitoring of chronic conditions can provide information about long-term trends in patient status, offering the possibility of intervention before chronic conditions become acute illness. Detecting acute conditions requires the system to be able to generate real-time alerts or alarms for patients, 
caregivers, and healthcare providers. Numerous RPM systems that are capable of providing real-time alerts for a variety of acute conditions such as falls, problems with driver alertness, seizures, and heart attack, have been investigated [46][76][77][78]. The characteristics of these systems, known as real time operating systems, are described in detail in the next section.

\subsection{Real Time Operating System}

Operating Systems (OS) can be broadly divided into two classes: (i) General Purpose Operating Systems (GPOS) and (ii) Real Time Operating Systems (RTOS). There are several differences between RTOSs and GPOSs, but most notably members of the GPOS class use a 'fair scheduling' algorithm process management system, whereas members of the RTOS class utilize a 'pre-emptive scheduling' system. The fair scheduling algorithm ensures that all tasks get a fair share of execution time; they do not take into account the priority of the task being executed. This is done to provide high system throughput, but obviously at a cost: specifically, fair scheduling can result in higher priority tasks getting delayed as they wait for lower priority tasks to finish. The priority-based scheduling system of the RTOS class, on the other hand, pre-emptively schedules higher priority tasks ahead of lower priority tasks, ensuring higher priority tasks are not starved of CPU time.

The RPM system may be required to monitor a number of different signals transmitted by a variety of sensors in a vehicle, the home environment, or worn directly on the body and transmitted through a mobile device (such as in a wireless Body Area Network (BAN) or wireless sensor network). Some commonly utilized sensors include 
GPS, the electrocardiogram (ECG), the electroencephalogram (EEG), the electromyogram (EMG), accelerometers, pulse oximeters, infrared sensors, video cameras, microphones, and pressure mats [79]. Sensor sampling rates may vary (typically from as low as $1 \mathrm{~Hz}$ to as high as $1 \mathrm{MHz}$ ) depending on the sensor used, but not all of these signals may be critical for generating real-time alarms [79]. It is known that an RTOS can guarantee predictable performance for the completion of high priority tasks, even under significant processing load in an environment requiring the management of many simultaneous tasks [80]. It has also been shown that an RTOS can be useful in the management of complex sensor networks such as an RPM system [81][82][83], and in environments where sensor input and decision making must be tightly coordinated, such as walking robot control systems [84].[81][82][84][83]. This means that, for an RPM system that employs an RTOS, any sensor measuring a critical physiological signal (such as an ECG monitor) or any process that is capable of generating an alarm during an emergency will always be processed in time every time.

\subsection{Home Monitoring}

As noted in Section 2.3, measurements of driving performance should be taken in with the context of overall health status. Older adults who have difficulty performing basic activities of daily living, or who are showing signs of worsening chronic illness may be at an increased risk of negative outcomes on the road. It has been shown that inhome monitoring can be used to obtain a large amount of information about the health status of an individual. For example, monitoring sleep activity and duration in the bedroom can provide information about sleep quality and overall mobility. This is 
important as poor sleep quality is an important predictor of a host of disorders such as heart disease [85] and diabetes [86], and increased time in bed is a sign of fatigue and reduced mobility, both of which are predictors of worsening health [87]. Many ADLs and IADLs, such as cooking, eating, and cleaning (sweeping, mopping, doing the dishes), can be monitored by observing kitchen use. A person who is unable to prepare meals to feed themselves is unlikely to be able to live entirely alone, so monitoring use of the kitchen is an important indicator of independence. In addition, the kitchen contains many potential sources of immediate danger in the home environment, and it is known that people with cognitive impairment are at a higher risk of fires caused by negligence when cooking. Bathroom use is especially important for monitoring health and independent living. Bathing disability - i.e. people needing help in the bath - is a sign of increasing dependency overall, and heralds overall health decline [88]. Also, failure to flush the toilet is known as a sign of dementia progression and increasing use of the toilet is a correlate of many common illnesses (e.g. diarrhea, urinary tract infection) [89].

While in-home monitoring using ambient sensors can provide extensive information about the health status of an individual, there are a number of difficulties to consider in doing so. Chief among them is the issue of patient privacy. An in-home monitoring system must not collect video data, especially in locations where privacy should be expected, such as the bedroom and bathroom. Most patients are be understandably unnerved by the possibility of direct observation of their homes [90], so instead of recording their activities with video, the monitoring system must instead rely on inferring activity indirectly based on the activation of other sensors. 
Our research group has been investigating in-home monitoring for some time. There has been research done that focus on a variety of areas in the home, but in recent years most of our efforts have focused on monitoring people in bed using pressure sensitive mats. Of particular interest of late is the idea that change in balance and mobility has been shown to be an important predictor of a change in health status, and that this effect holds even people who are confined to bed [19]. Currently, work is ongoing with the aim of discovering a method for predicting falls [91], for scoring the Hierarchical Assessment of Balance and Mobility (HABAM) [92], and for monitoring sleep quality and sleep apnea [93].

\subsection{Conclusion}

This chapter described how the ageing of the population has provided the impetus to develop RPM systems. It provided a review of existing RPM systems and demonstrated that many are not suitable for meeting the needs of the majority of elderly people, showing that further research is needed to improve RPM usefulness and costeffectiveness. Additionally, this chapter provided a basis for understanding the necessity of including an RTOS in any RPM system that can generate real-time alarms. Finally, it gave a background for home and mobile RPM systems, and explained the importance of each in monitoring high-level body functions, such as mobility and balance. 


\section{Chapter 3:}

\section{Equipment Setup and Experimentation}

\subsection{Introduction}

Although the RPM system created for this thesis was designed to be adaptable for use in a wide variety of environments (the details of its architecture and implementation will be presented in Chapter 4), this document focuses primarily on its deployment in a vehicle. One key concern when implementing a system that might be used for protecting drivers (such as a seizure detection algorithm) is its reliability: if the system cannot be counted on to make timely decisions, then it will not be of any use. Even if a particular RPM setup does not intervene in an emergency, it is important that the capability exists in the case that the system is expanded to include such requirements. Therefore, one of the experiments performed for this thesis aimed to test its reliability (detailed in Chapter 5). The second experiment aimed to begin the process of determining driver aptitude through identifying patterns in driver acceleration waveforms (presented in Chapter 6). In particular, this chapter describes the specific hardware setup for each experiment performed: the real-time operation of the RPM system and the accelerometer calibration experiment. It also provides details of the data collection procedures used in each test. 


\subsection{Equipment Setup}

The hardware used in each experiment was similar, but with differing sensor layouts. Additionally, the accelerometer calibration experiment was performed "offline", so the central node was not used. This section details the specific hardware layouts for each experiment performed.

\subsubsection{Real Time Monitoring}

In order to test the real time performance of the system, it was configured in the format shown in Figure 1 (a high-level sequence diagram of the software written for each component of the system is available in Appendix A). The application that was written to access the sensor array on the smart phone (used in the accelerometer calibration

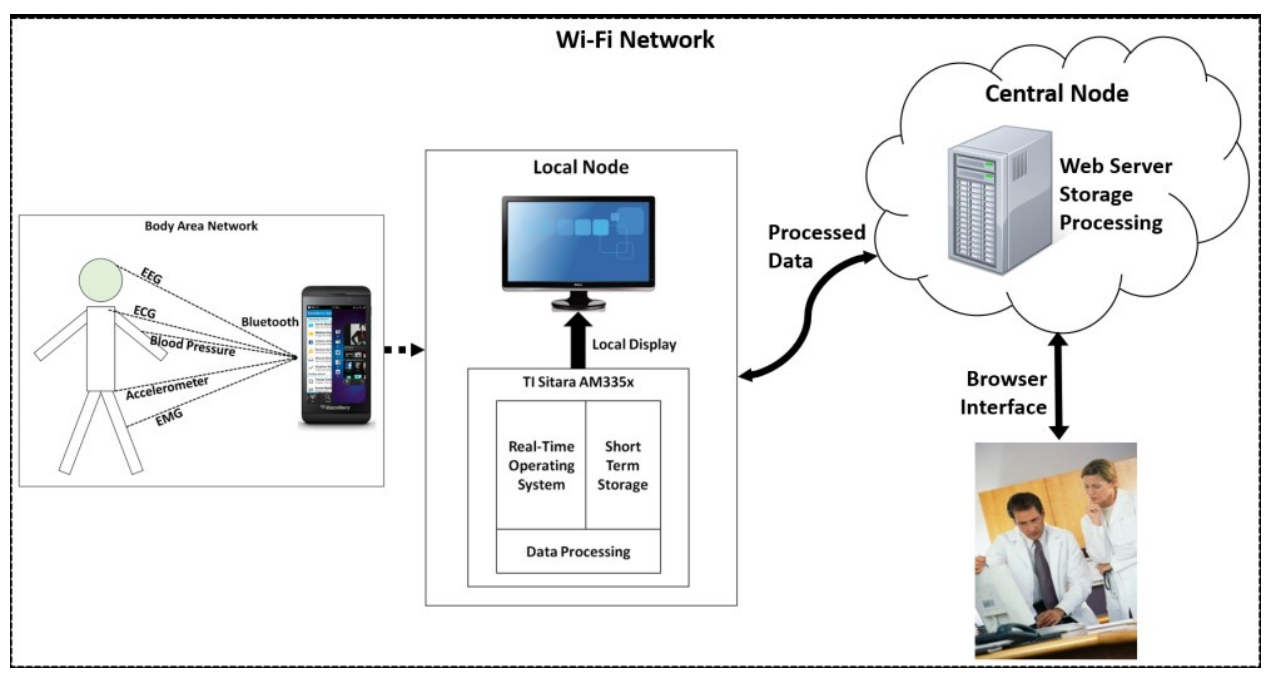

Figure 1: Real time monitoring equipment setup.

The RPM system used in the real-time monitoring test. The sensors were simulated on the phone and transmitted to the local node via Wi-Fi where they were processed and sent to the central node (along with timing data) for storage and further analysis. 
experiment described in the next section) also included an additional five simulated sensors (detailed in Chapter 5) that were then connected to a microcontroller (Texas Instruments Sitara AM335x Starter Kit, model TMDSSK3358) acting as the local node via a TCP/IP connection over a standard $802.1 \mathrm{n}$ Wi-Fi network. This local node communicated with a node.js Javascript webserver on a Dell computer acting as the central node via the same Wi-Fi network. It made use of HTTP, sending POST requests to push timing data generated based on the input form the smart phone simulated sensors. The central node both hosted the server and acted as the web interface to observe the incoming streams of data.

\subsubsection{Accelerometer Calibration}

For the accelerometer calibration experiment, sensors to measure velocity and acceleration were deployed in a test vehicle (Bruce Wallace's personal vehicle) in order to collect the data used in the accelerometer calibration and driver assessment experiment detailed in Chapter 6. For measuring velocity, a Candrive Persentech OttoView-CD sensor [59][60][94] was provided by the Candrive project and deployed. It will be discussed in further detail in Chapter 4, but it was primarily used to provide two measures of velocity:

- GPS: The Global Positioning System directly measures the position of the vehicle and it also provides a measure of velocity through calculation of the change in position over time. 
- OBDII: The On Board Diagnostics Version II interface has been standard on all North American vehicles since 1996 and provides access to the dashboard vehicle velocity measurement.

To measure the acceleration, three identical Blackberry Z10 smart phones, each containing a three axis accelerometer, were used along with an application [95] that recorded the accelerometer readings. The accelerometers were sampled at $40 \mathrm{~Hz}$. Three smart phones were used instead of only one so that multiple recordings of each of the test cases were available. During testing, each smart phone was positioned so that the forward motion of the vehicle was aligned with only one axis of its accelerometer. The phones were rotated periodically throughout the testing so that the calibration of each axis could be assessed.

\subsection{Data Acquisition}

As described above, two experiments were performed to independently test different contexts in which the system might be used. The first examined the real-time system itself, and the second measured movement in a car.

\subsubsection{Real Time Data Processing}

The first experiment involved testing the RTOS itself by gauging the system's ability to respond quickly under high processing load. Five sensors were simulated on a smart phone, and each was configured to transmit a 16-bit white noise signal to the local node at a rate of $1 \mathrm{KHz}$. The local node collected data in a separate thread for each simulated 
sensor, and, to simulate a high processing requirement, it performed a number of executions of a short-term power calculation every 1000 samples. The short-term power calculation was chosen because it is a common 'first step' type calculation and used in many signal processing applications. A timestamp was generated and logged before beginning and after completing processing on each 1000 sample 'batch' of data. The resulting dataset contained five comma separated value files containing the completion time for the short-term power calculations on the data from each simulated sensor.

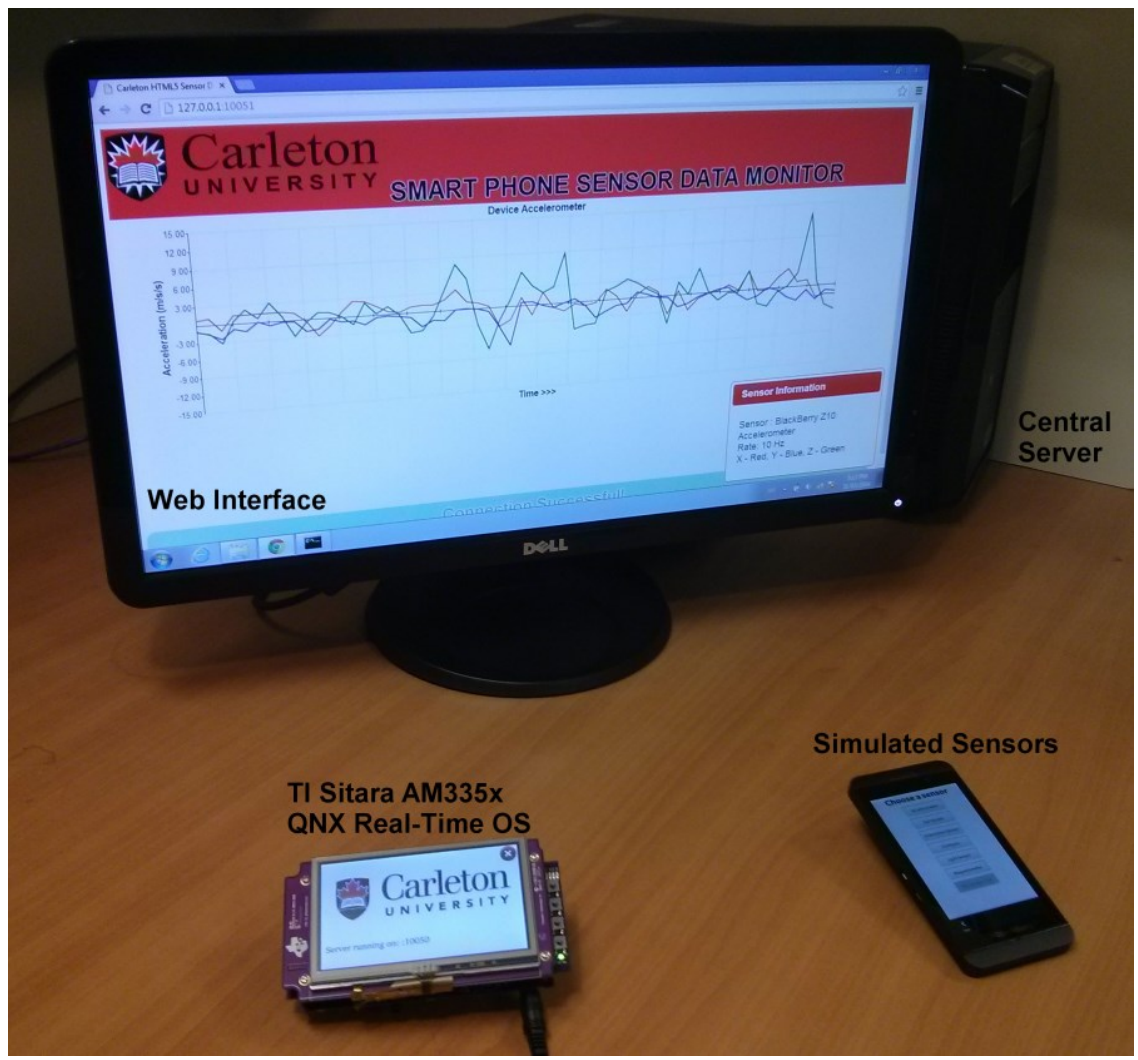

\section{Figure 2: Real time monitoring experiment equipment setup.}

There are five sensors simulated on the smart phone, each transmitting 16 bit data at a sampling rate of $1 \mathrm{kHz}$. The local node, running on a TI Sitara AM335x Starter Kit microcontroller, receives the data from the simulated sensors, and generates completion timing data for a number of short-term power calculations. Both the raw data and the timing data are then passed to the central node (the computer visible in this image) and stored. 


\subsubsection{Driving Data}

Approximately two hours of driving data were collected using the Candrive sensor and the three smart phone accelerometers. Acceleration data was collected for the $\mathrm{X}, \mathrm{Y}$, and $\mathrm{Z}$ axes, and was stored on each phone at a sampling rate of $40 \mathrm{~Hz}$ and organized into comma separated values files. Three smart phones were used so that the default calibration of each accelerometer could be effectively assessed and later corrected. The phones were arranged in a "stacked" configuration and mounted on a standard smart phone car mount with their top sides (positive $\mathrm{Y}$ axis) oriented toward the front of the vehicle, as shown in Figure 3. At specific points during the acceleration testing, the bottom two smart phones were rotated 90 , and 180 degrees so that the calibration of both directions of the $\mathrm{X}$ and $\mathrm{Y}$ axes could be assessed (as shown in Figure 4). The resulting dataset contained data from approximately one hour of normal driving and one hour of acceleration testing. During acceleration testing, the car was accelerated from $0 \mathrm{~km} / \mathrm{h}$ to a top speed of 60,70 , or $80 \mathrm{~km} / \mathrm{h}$ repeatedly over the same length of rural road. In addition to the three smart phone accelerometers, velocity data was collected from the vehicle's On-Board Diagnostic (OBDII) port, and position data from an on-board GPS system. These two datasets were used as gold standard measures to compare to the accelerometer's performance when calculating velocity and position. 


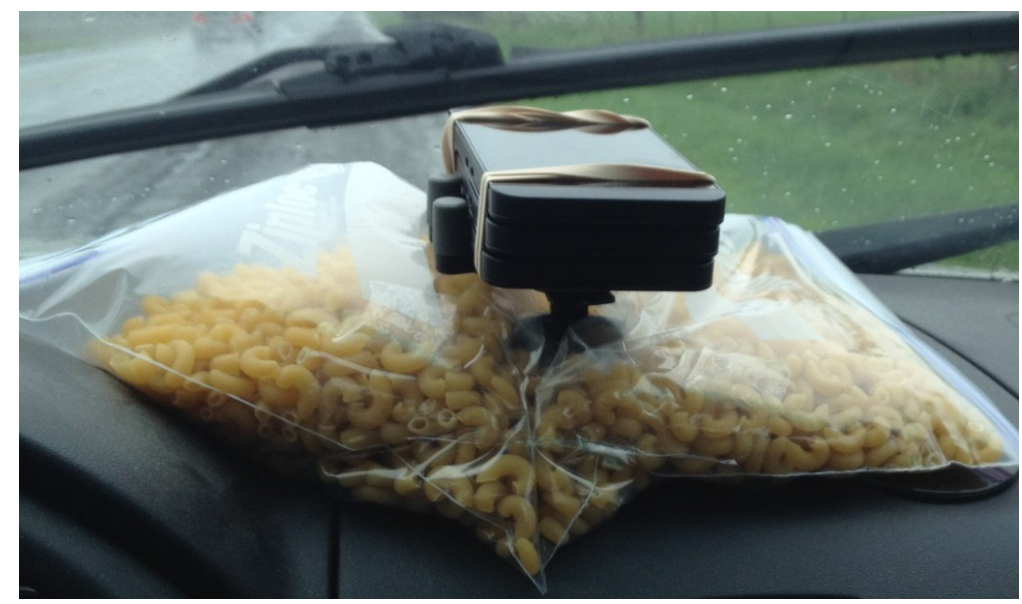

Figure 3: Accelerometer stack with top sides (positive $Y$ axis) oriented toward the front of the vehicle.

The primary setup for collecting driving acceleration data from the three smart phone accelerometers. The first set of acceleration profiles were collected with all three phones oriented so their top sides (positive Y) were oriented toward the front. The second set of accelerations profiles were collected with the top of the top phone and the bottom of the bottom two oriented toward the front. Macaroni bags used as support and to reduce vibration interference.

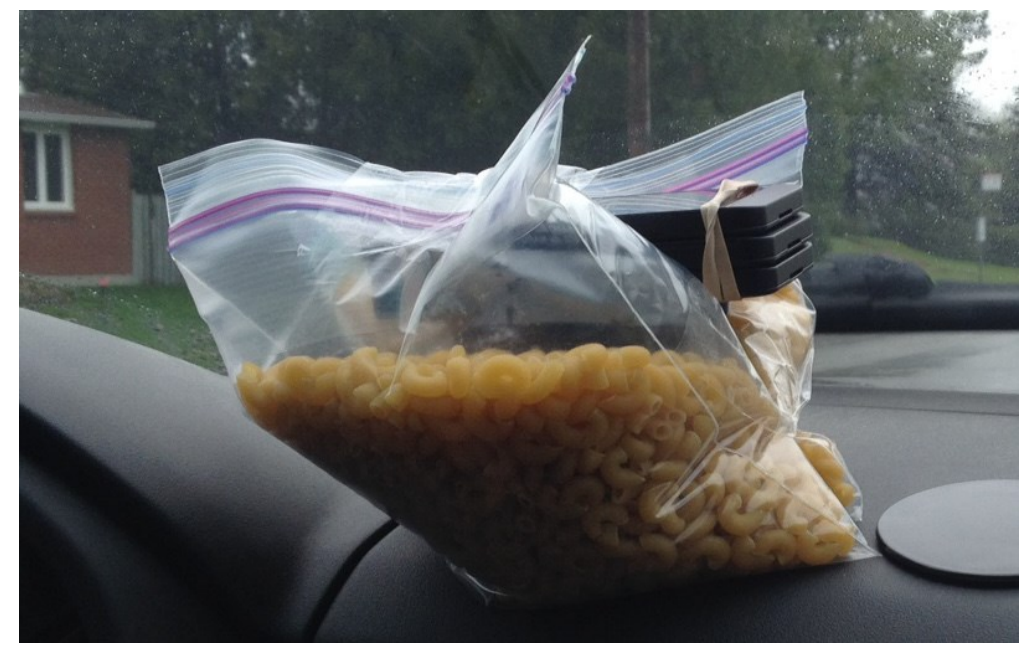

Figure 4: Accelerometer stack with left sides (positive $X$ axis) oriented toward the front of the vehicle.

The secondary setup for collecting driving acceleration data from the three smart phone accelerometers. The third set of acceleration profiles were collected with all three phones oriented so that their left sides (positive X) were oriented toward the front. The final set of acceleration profiles were collected with the left side of the top phone and the right side of the bottom two facing the front. Macaroni bags used as support and to reduce vibration interference. 


\section{Chapter 4:}

\section{Remote Patient \\ Monitoring System Design and Implementation}

\subsection{Introduction}

The RPM system introduced in the previous chapter was designed to be highly generalizable so that it could be used to meet the many, varied needs of an elderly population. This chapter discusses the design and implementation of that RPM system. Many systems that are capable of remote sensor monitoring have been created, and there is a wealth of information on the topic and a variety of basic architectures to choose from. With that in mind, the opening section of this chapter describes the different RPM system designs considered, then provides details of and justification for the one that was chosen. Additionally, the experiments mentioned in Chapter 3 require certain sensor configurations, so this chapter also describes the sensors used in those experiments. Finally, it describes how each other component of the chosen architecture was implemented both in software and in hardware. 


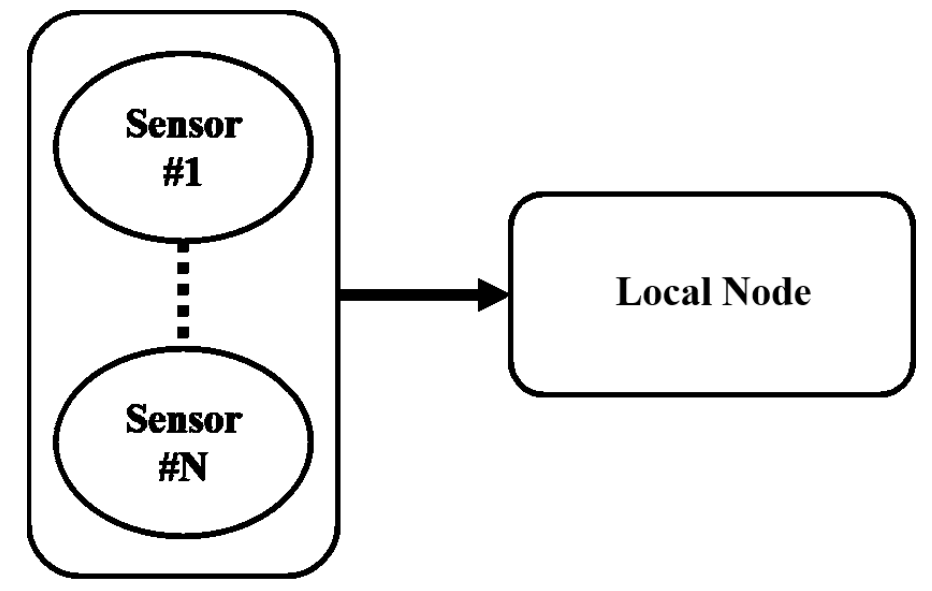

Figure 5: Local RPM system design

This RPM system design consists of one or more sensors connected to an on-site data collection and storage device. It is commonly used in research settings, and has been employed by both the Candrive study and in research on bed- and floor-mat sensors.

\subsection{System Design}

While there are a wide range of specific RPM system architectures, many fall into three high-level categories. These three categories are referred to here as 'local' (shown in Figure 5), 'centralized' (shown in Figure 6), and 'centralized local' (shown in Figure 7). Perhaps the most common basic design is the local only architecture. This architecture is used by our group for research with the bed and floor mats, and by the Candrive research group for collecting in-vehicle data. It is the easiest to implement, simply consisting of one or more sensors connected directly to a local data collection device (often a laptop or specialized hardware). The obvious drawback of this system is that, because there is no way for it to communicate with any remote device or individual, it offers only a limited capacity for real time monitoring and intervention, and no possibility of generating remote alarms. It also requires a site visit in order to collect data for 
analysis and to perform maintenance or to ensure proper operation of the sensors. This design is commonly used in research, both because it is so easy to implement and because there is often no need for anything more complex while still validating and exploring the data. It is also employed for monitoring chronic diseases, such as diabetes, where emergency intervention is unlikely and the patient can manage their condition with

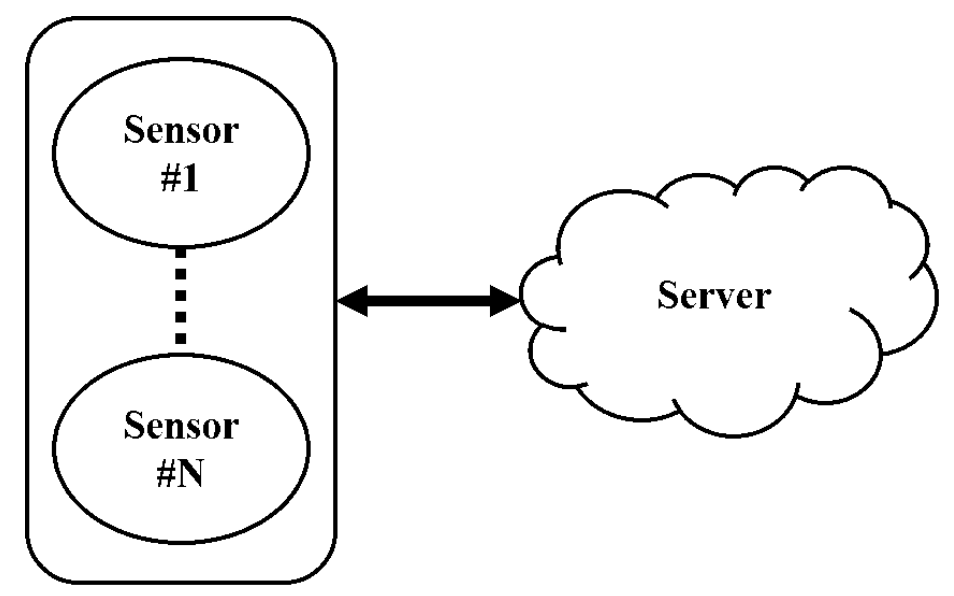

Figure 6: Centralized RPM system design

This RPM system design consists of one or more sensors connected to a central, off-site server for data collection and storage. This design is more complex to implement than the previous one, but can offer advantages for long-term monitoring, since a site visit is not required for data collection.

relative ease.

Centralized systems (Figure 6) are more complex to implement and require an internet connection, but are very useful in research for long-term data collection, or in situations where it is not feasible to regularly perform site visits to collect data. In these systems, sensor output is fed directly to a central server over the internet where it can be stored an analyzed. These systems are also advantageous in that they offer the possibility of monitoring health status in real-time. However, as will be clarified later in this chapter, if the system is to implement any real time interventions or alarms, this architecture will 
not be sufficient. Further, in the case of an in-vehicle RPM system, interruptions in network service are almost a guarantee, so this architecture will not be suitable.

Finally, the centralized local architecture combines both of the previous architectures by connecting the local data collection device to a central server. This architecture has a number of advantages over the others in that it combines robustness in the face of inconsistent network connectivity with the data collection and communication capabilities offered by a central server. Further, since this system offers on-site processing it is capable of reducing the bandwidth needed to transmit data to the central node through raw sensor parsing and analysis. This was the design chosen for this RPM system implementation. The block diagram in Figure 7 shows a high-level view of the system layout, which is built around the local node, a microcontroller running an RTOS connected to the Internet. The sensors may be connected to the microcontroller via a wireless network connection, a wired network connection, or Bluetooth. Data received by the microcontroller are analyzed and forwarded through the network to the server, which logs the data and displays them in a browser window. Each of these components will be elaborated on in later sections of this chapter. A high-level sequence diagram of the software implementation is presented in Appendix A. 


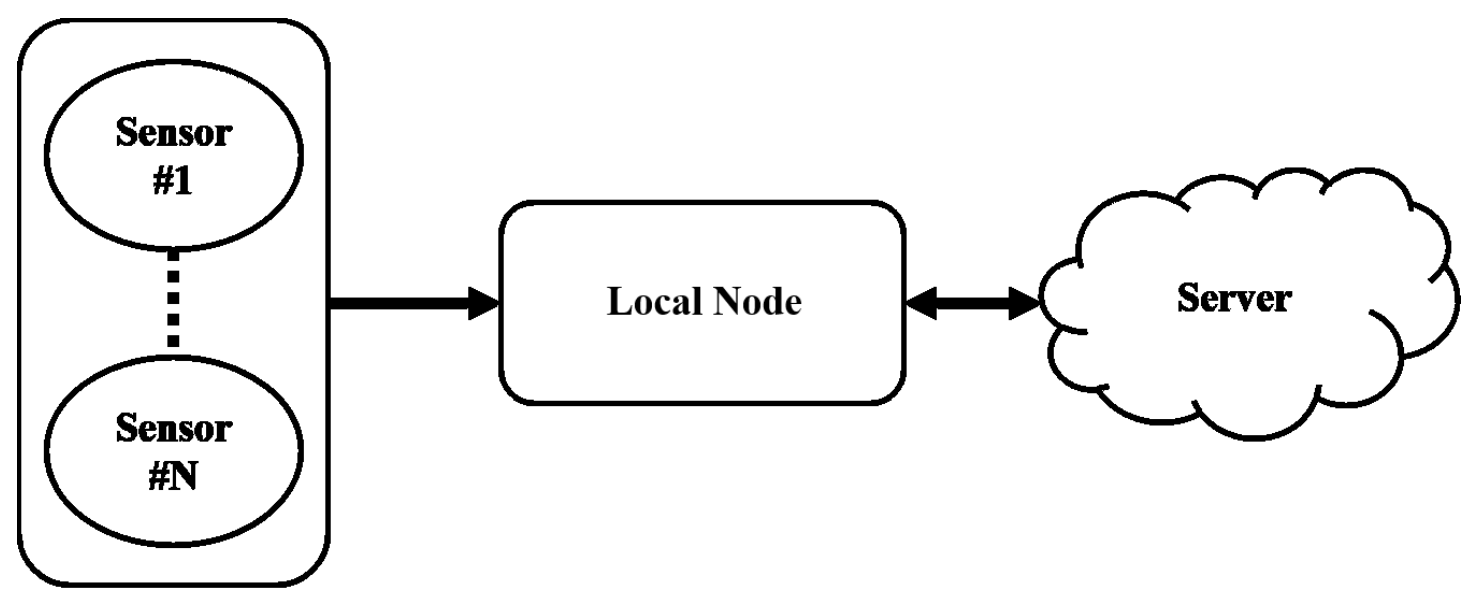

Figure 7: Centralized local RPM system

This RPM system design is a combination of the previous two: it involves the use of a local node for short term storage and direct interaction with the sensors, and a central server for long term data storage and analysis.

\subsection{Architecture}

Figure 8 shows the high-level architecture chosen for the RPM system. It consists of a number of sensors used to monitor the patient; an on-site local node that accepts sensor input and performs data analysis and short-term storage; an off-site central node for long-term data storage and further data analysis or other computationally expensive work; and a web-server that provides a graphical user interface for patients and clinicians to monitor the status of the patient. 


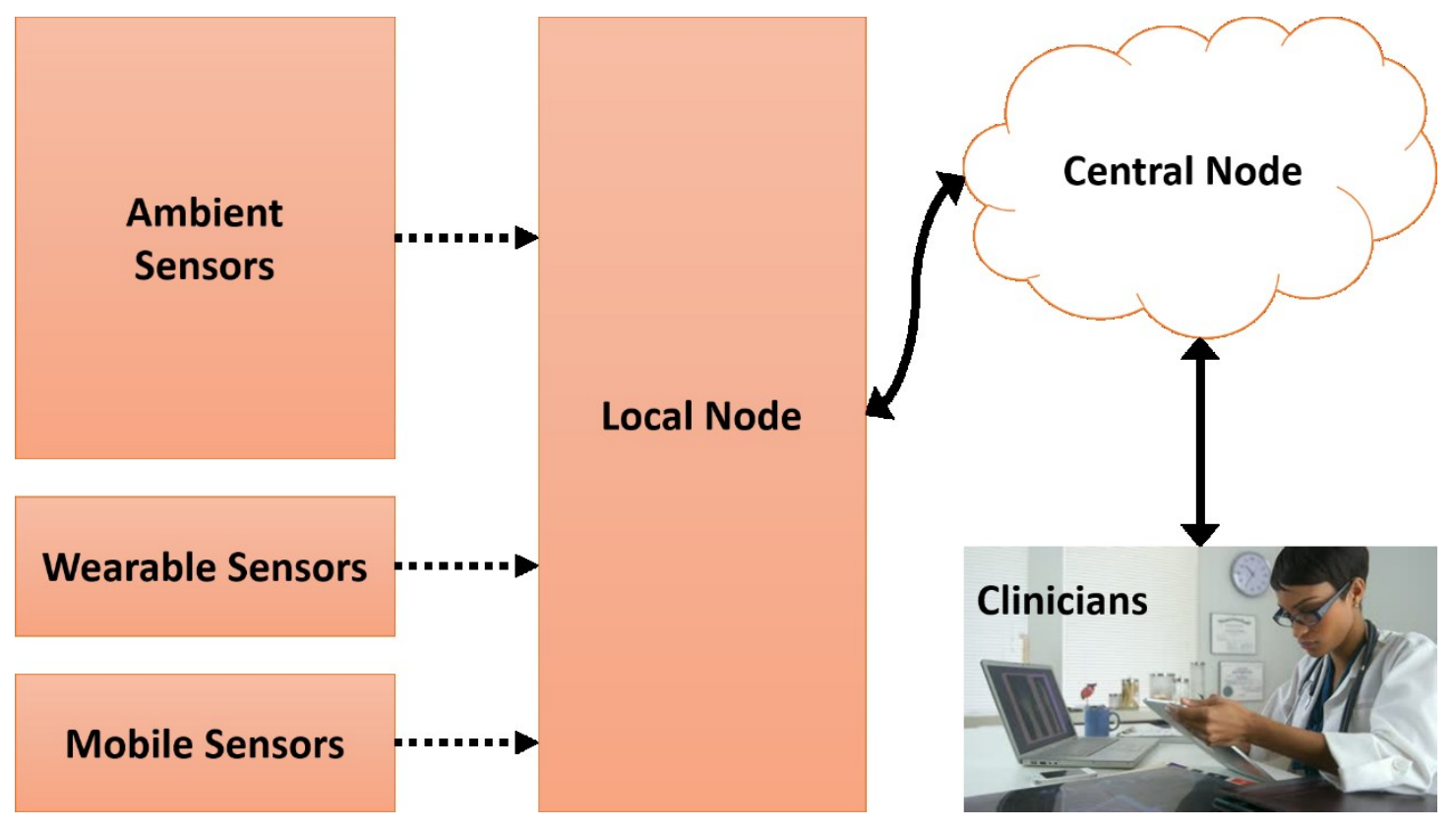

Figure 8: Architecture of an RPM system

The implemented RPM system is built around a dedicated local node that captures sensor data, performs basic filtering and high priority analysis, then passes the parsed data on to a separate central node to be stored and further analyzed. Clinicians, patients, and family members can access the captured data through an interface with the central node.

\subsubsection{Sensors}

Broadly speaking, there are three categories of sensors: (i) wearable sensors, (ii) sensors embedded in a mobile device, and (iii) 'ambient' sensors that are installed throughout the environment (such as a home or vehicle) to monitor the patient's movements and ability to perform activities of daily living. Each category has a number of advantages and disadvantages: wearable sensors can often provide more precise information about direct measures of patient health, but they can be uncomfortable or irritating to wear, rely on batteries for power, are weight and size limited, and require patient cooperation in order to be effective. In many settings, sensors embedded in a 
mobile device such as a smart phone are as capable of measuring physiological data as are wearable sensors, but with a number of advantages: they are not affixed directly to the body, they have substantial on-board processing power, and they can collect and send data wirelessly even without access to a wireless network. Unfortunately, they also retain a number of the same drawbacks as wearable sensors, particularly in that they are battery operated and require patient cooperation. Ambient sensor systems, on the other hand, require no patient cooperation and, for most practical purposes, are not limited by weight, size, or power source, but as mentioned previously, they cannot typically measure physiological activities directly, so they must extrapolate patient status through indirect measurement. Importantly, too, ambient sensor systems fail altogether when the patient moves out of their range. Sensors and sensor types can work together to provide a clearer understanding of the overall status of the patient. For instance, when monitoring driving it could be useful to know not just the patient's movements and the state of their health, but also the weather conditions and the state of the vehicle (such as through internal/external temperature sensors).

\subsubsection{Local Node}

The local node collects, processes, and temporarily stores the data generated by these three types of sensors before forwarding the processed data to a central server for long-term storage and analysis. It can be implemented using a custom built hardware platform, a PC, a mobile device such as a tablet or a smart phone, or even installed directly into a vehicle as part of its on-board OS. If real-time alarm generation is required, then all processing involved in the alarm generation must be performed at the 
local node. Processing real-time critical data locally allows the RPM system to generate alarms even when network is congested or down, unlike when all the data processing is done at the central node. In such cases, alarms can be generated using SMS, phone calls etc. Since not generating alarms in a timely manner can have catastrophic results for a patient, the real-time performance of the local node is a critical design parameter. The easiest, most reliable way to ensure this is to use an RTOS on a dedicated local node (further, since creating an RPM system to meet the needs of older adults is the focus of this thesis, and they are considerably less likely to make use of smart phone technology, it is important to have a local node that can stand alone). In a vehicle equipped with an on-board RTOS, the vehicle itself can act as the local node through its on-board computer, communicating with the central node through a 3- or 4G data connection.

\subsubsection{Central Node}

The central node is the major hub for communication between patients, users, and local nodes. It is used for long term storage of event logs and sensor data, for low priority data processing, and for chronic illness trajectory tracking. Additionally, it can be used as a central location for all patient information, and provide a graphical user interface for patients, clinicians, and family members to keep track of patient status. Each central node can serve many local nodes, and can communicate directly with each to push updates, diagnose technical issues, or even allow instantaneous communication with the patient in the event an emergency is detected. 


\subsection{Implementation}

The implemented system consisted of a smart phone, a microcontroller running an RTOS, and a laptop computer hosting the central node web server. This system is a prototype, but is expandable to include any other variety of sensors and trivially upgradable to take advantage of dedicated server hardware. Any sort of direct installation in a vehicle would require further development and partnership with industry, but this is not an issue for two reasons: the system is designed to be deployed in the home, and the microcontroller running the local node requires sufficiently low power to operate on the typical 12 volt vehicle power supply, so it can be installed separately.

\subsubsection{In-Vehicle Sensor}

The Candrive Persentech OttoView-CD Sensor (Figure 9) is a custom-made in-vehicle recording device that is installed directly to the vehicle's OBDII port, which has been standard on all vehicles in North America manufactured since 1996. It captures information from the vehicle about the time and date, the speed, and the distance travelled, as well as a number of engine parameters. In addition, each sensor comes with a GPS antenna, and, optionally, a Radio Frequency Identifier (RFID) system if the vehicle has more than one driver (with an RFID tag added to each driver's keyring to allow differentiation between each). A full list of collected parameters is shown in Table 1. Data is stored on an SD card at a rate of $1 \mathrm{~Hz}$. 
Table 1: Data collected by the Candrive Persentech OttoView-CD sensor

\begin{tabular}{|c|c|c|}
\hline OBDII Port & GPS & RFID System \\
\hline Date & Latitude & Driver ID \\
\hline Time & Longitude & \\
\hline Velocity & Calculated velocity & \\
\hline Tachometer & & \\
\hline Engine temp & & \\
\hline External temp & & \\
\hline
\end{tabular}

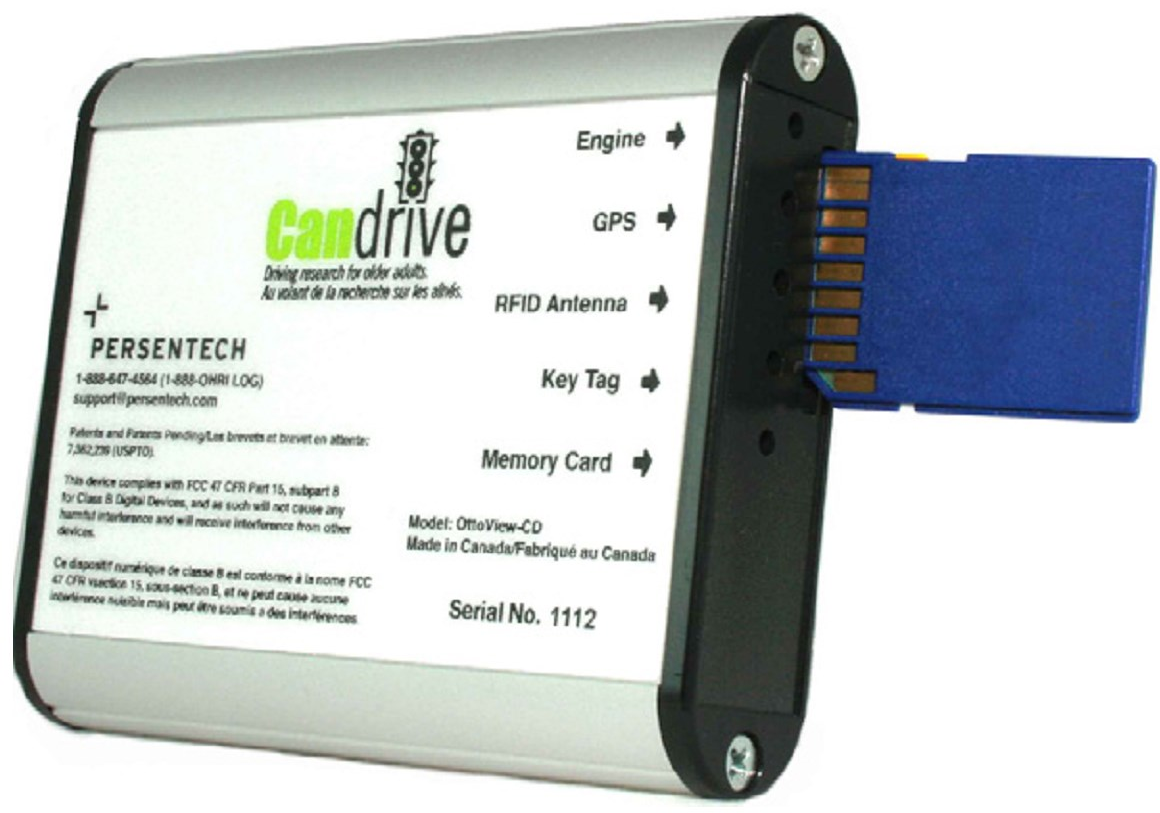

Figure 9: Candrive Persentech OttoView-CD sensor [59]

This sensor records data from the vehicle's OBDII port, an installed GPS antenna, and optionally from a driver identification RFID system. Data is stored locally on an SD card at a rate of $1 \mathrm{~Hz}$. SD cards are collected, transferred to a secure hard drive and replaced at three to four month intervals. 


\subsubsection{Sensors}

For the experiments performed in this research, data was collected from the Candrive Persentech OttoView-CD sensor described in the last section as well as from the sensor array built into a Blackberry Z10 smart phone (Figure 10). The sensor array consists of an IMU (comprising a three-axis accelerometer, a gyroscope, and a magnetometer), a light intensity sensor, a compass, a temperature sensor, two cameras, and a microphone. In our studies we chiefly relied upon the components of the IMU, and the accelerometer specifically.

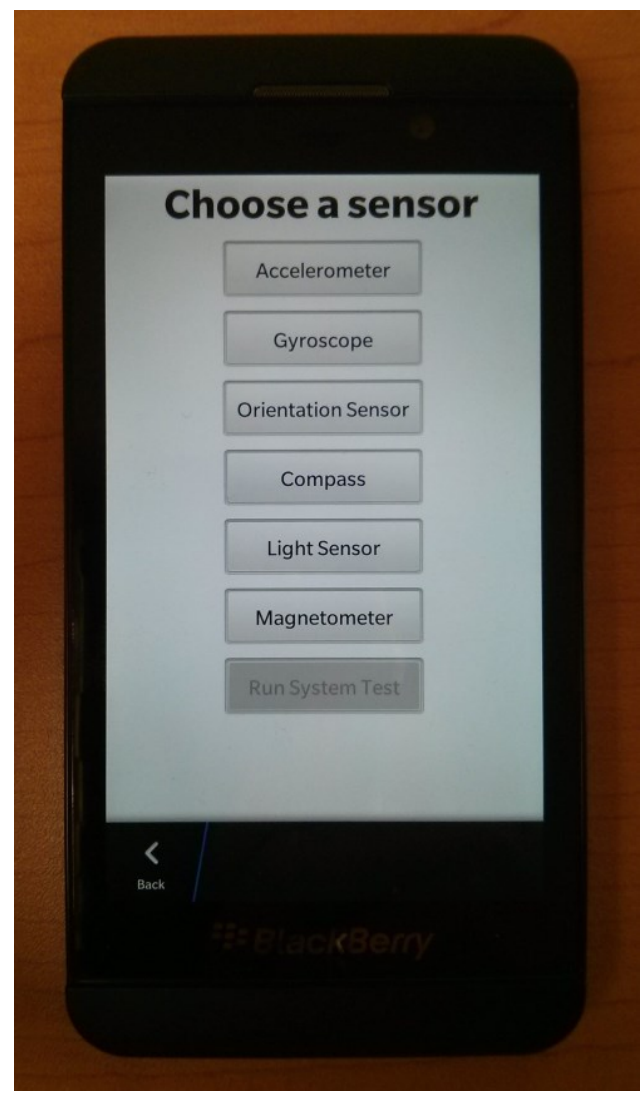

Figure 10: The smart phone sensor array

This application transmits data from the built sensor array to the local node over a wireless network connection. If no network connection is available, data is stored locally. All sensors can be activated simultaneously, or one at a time. 


\subsubsection{Local Node}

RPM systems that are designed to generate alarms in the case of an emergency must be able to guarantee that they can do so immediately upon discovering a problem. GPOSs are not up to this task, especially when under heavy processing load (as is often the case with devices such as personal computers, tablets, and smart phones). In the context of driving, an automobile power supply is typically somewhat limited, so the processing power of any local node will necessarily be limited too. Additionally, devices used for purposes other than remote monitoring activities run the risk of potentially significant downtime should some other process crash the system. The local node was therefore implemented as a separate device with dedicated processing hardware and the QNX Neutrino RTOS. The hardware chosen was the Texas Instruments Sitara AM335x Starter Kit microcontroller (model TMDSSK3358, Figure 11). It utilizes an AM3358 Cortex-A8 processor, with $256 \mathrm{MB}$ of DDR3 memory and a $\mu$ SD card reader that offers flexibility in short to medium-term data storage. The device is equipped with two Ethernet ports, two USB 2.0 ports (one standard USB port, and one micro USB port), built-in Wi-Fi and Bluetooth connection capabilities, and an attached LCD touch screen display [96]. It was chosen because it is a comparatively low-cost device that meets the requirements for running the QNX Neutrino operating system. The QNX Neutrino operating system is a real-time operating system that has been used for a variety of timecritical applications such as car systems and medical device control, and so has been designed to meet extremely strict real-time requirements [97]. It is described in further detail in chapter 5. 
The software application on the local node was written in $\mathrm{C}++$ using the $\mathrm{Qt}$ application framework. This application framework was chosen because it is largely platform independent, to allow the local node software to be installed on a variety of devices, including smart phones, personal computers, microcontrollers (as in this application), or even some car operating systems. It employs an event-driven model, in

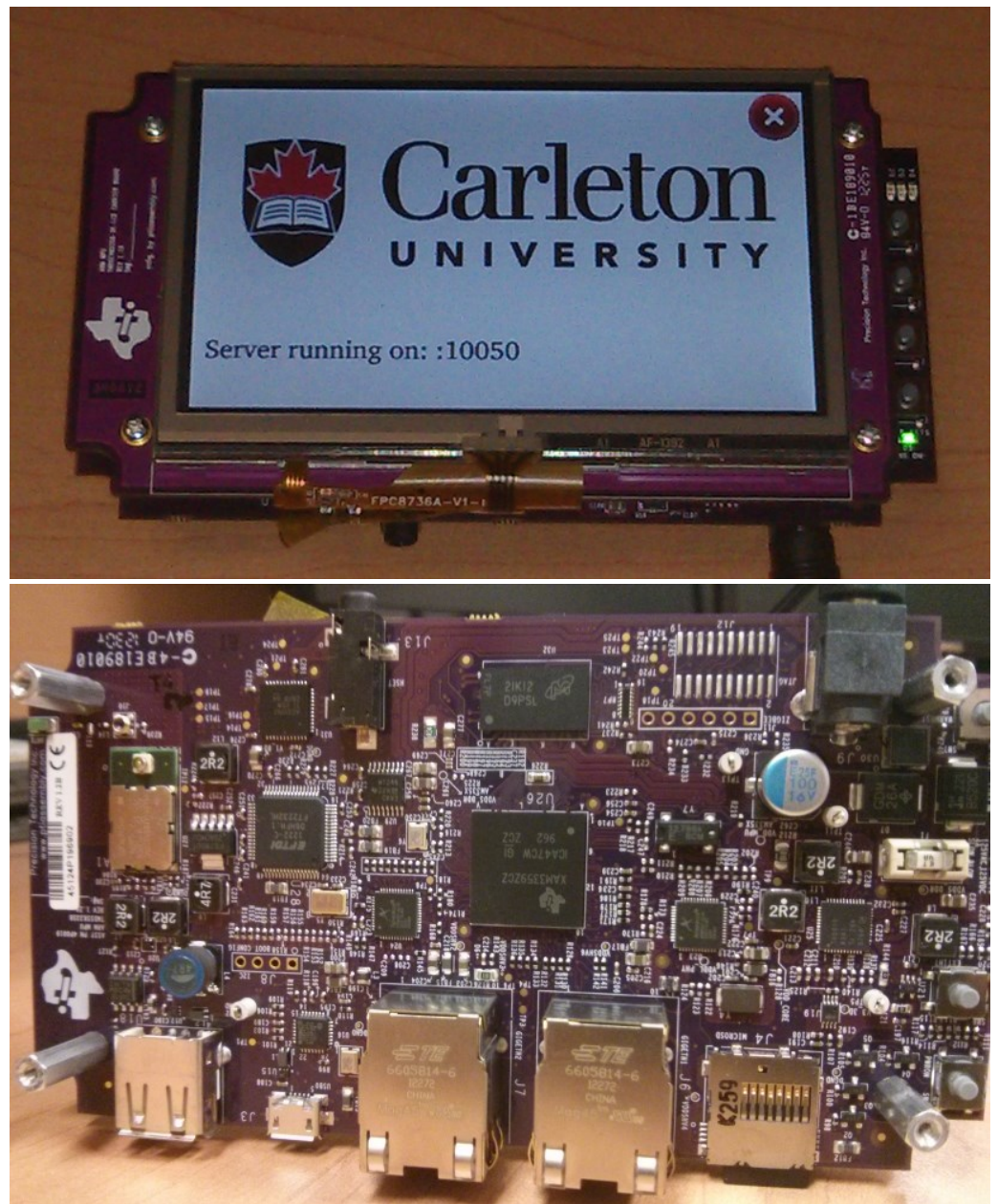

Figure 11: The local node (top and bottom)

The local node selected for this implementation was the Texas Instruments Sitara AM335x Starter Kit microcontroller running the QNX RTOS. The application receives, temporarily stores, and processes data from the sensors connected to it before passing the processed data to the central node along with timing and log information. 
which each component of the server software is connected via event emitters and consumers. Each connected device can transmit data asynchronously, enabling easier multi-threading and saving the server software from ever wasting processing time in a waiting state. A single, main server object serves as a hub for all incoming and outgoing network traffic. It employs a TCP socket to listen for sensor connections and communicates with the central node via HTTP. These connection types were selected because TCP guarantees reliable, ordered, error-free delivery of a continuous stream of data. It accomplishes this performance at the cost of a larger data overhead and somewhat increased latency than other protocols such as UDP. This is desirable because, although the connections between the local node and sensors are expected to be stable, they will vary in quality (especially on a wireless network), and it may be critical that all sensor data is received and in the correct order for accurate analysis. HTTP is built on top of TCP, and so contains an even larger data overhead, but is more robust than TCP in the face of an unstable network connection.

Sensor data can be analyzed on the local node in order to perform high priority tasks (such as generating alarms in case of falls or environmental hazards), to perform real-time context awareness calculations, to reduce the volume of data sent to the central node, or to covert data from a raw format to known units to make further analysis easier. Upon establishing a connection with a sensor, the server begins buffering incoming data and dividing it into non-overlapping 'windows' of predetermined length, which can then be processed, stored, or transmitted to the central server for long-term storage and analysis. In this way, data can be more easily managed, and more efficiently transmitted than if it were handled byte by byte. Importantly, this also allows the real time processing 
threshold to be varied according to the sensor analysis priority. This useful advantage will be detailed in Chapter 5 .

\subsubsection{Central Node}

In this implementation, the central node consists of a web server running on a Dell computer using the Windows 7 OS. The web server is written in Javascript with the node.js and socket.io server frameworks. As with the local node, the software written for the central node uses an event-driven model. A number of local nodes can communicate with a single central node via HTTP, as described in the previous section. The central node receives status updates and parsed sensor data from the local node through HTTP POST requests. It acts as a central hub for patient information: it organizes and stores status and sensor data sent from the local node, and provides a web interface that allows remote users to connect and observe received data in real time. Stored data can later be analyzed to monitor driver habits or changes in driver signature if the system is installed in a vehicle, or, if deployed in the home or a hospital setting, to detect negative changes in chronic conditions, to track recovery from surgery, or to assess the effectiveness of new treatments. 


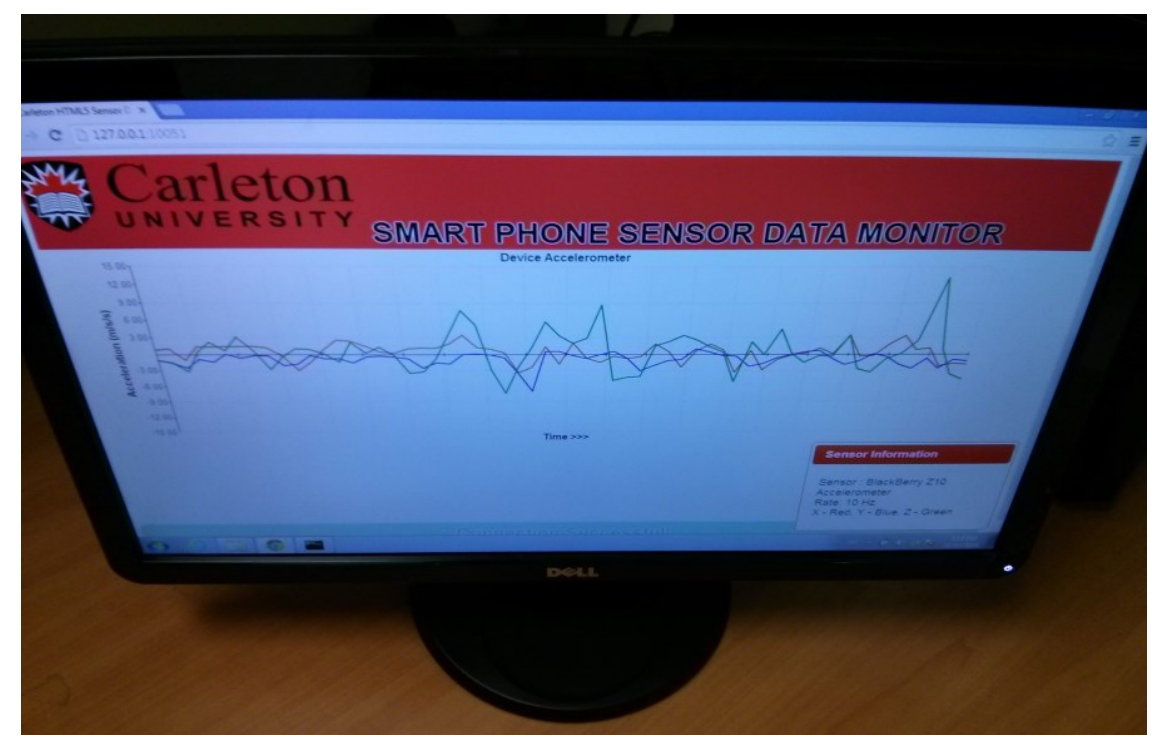

Figure 12: The central node

In this implementation, the central node consisted of a node.js web server on a Dell computer running the Windows 7 OS. The web server accepts processed data from the local node, stores it and hosts a web page to display it.

\subsection{Conclusion}

This chapter discussed the design and implementation of the RPM system. The design chosen was the centralized local architecture, in which one central node monitors a number of distributed local nodes, which have direct access to the sensor array. Despite being the most complex to implement, this architecture was chosen because it allows the system to manage sensor and analysis priorities, can perform local processing to reduce demand on bandwidth, and can reliably generate alarms in emergencies. Further, it is the most easily expandable, as adding/removing sensors or changing their functionality requires only a software update, which can be pushed to the local node from the server. 


\section{Chapter 5:}

\section{Real-Time Operating System}

\subsection{Introduction}

This chapter focuses on the effect of the RTOS used in the design on the RPM system as a whole. It opens by describing the real-time requirements of the RPM system, and expands upon the reason this is a critical design requirement when implementing any RPM system capable of generating alarms. It describes the analysis of the data collected from the simulated sensors in the real time monitoring experiment described in Chapter 3. Further, it explains the advantages of using an RTOS in general, and the QNX Neutrino operating system in particular. Finally, it demonstrates the reasons for choosing a dedicated processing unit in the home environment rather than a personal computer or general use mobile device.

\subsection{Real-Time Requirements}

Any RPM system that aims to do continuous, real-time monitoring needs to manage the requirement that the system be able to "keep up" with the rate of incoming 
Table 2: Sampling and data rates for various types of common biomedical measurement devices

\begin{tabular}{|c|c|c|}
\hline Measurement Device & Sampling Rate & Bit Rate \\
\hline 3 Axis Accelerometer, 16 bit @ $40 \mathrm{~Hz}$ & $40 \mathrm{~Hz}$ & $2 \mathrm{kbps}$ \\
\hline 24 Point Bed Pressure Mat, 12 bit @ $10 \mathrm{~Hz}$ & $10 \mathrm{~Hz}$ & $3 \mathrm{kbps}$ \\
\hline 72 Point Bed Pressure Mat, $12 \mathrm{bit} @ 20 \mathrm{~Hz}$ & $20 \mathrm{~Hz}$ & $17 \mathrm{kbps}$ \\
\hline 3 Lead ECG, 16 bit @ $500 \mathrm{~Hz}$ & $1.5 \mathrm{kHz}$ & $24 \mathrm{kbps}$ \\
\hline 12 Lead ECG, 16 bit @ $500 \mathrm{~Hz}$ & $6 \mathrm{kHz}$ & $96 \mathrm{kbps}$ \\
\hline Audio, 16 bit @ $20 \mathrm{kHz}$ & $20 \mathrm{kHz}$ & $320 \mathrm{kbps}$ \\
\hline
\end{tabular}

data, even in the face of high processing demand. The RPM system designed for this thesis was designed to be used under a variety of circumstances and to support many types of sensor, so it is important to make sure this requirement has been met. Sensors can transmit data at any rate, depending on their type, but those used in most remote monitoring applications typically sample at rates in under a few $\mathrm{kHz}$ (shown in Table 2). Depending on the input rate and the complexity of the required processing, real-time data are often not processed sample-by-sample, but rather in batches or "sample windows" as it arrives (shown in Figure 13). These sample windows determine the processing time threshold for real-time applications as described in Equation 1: 


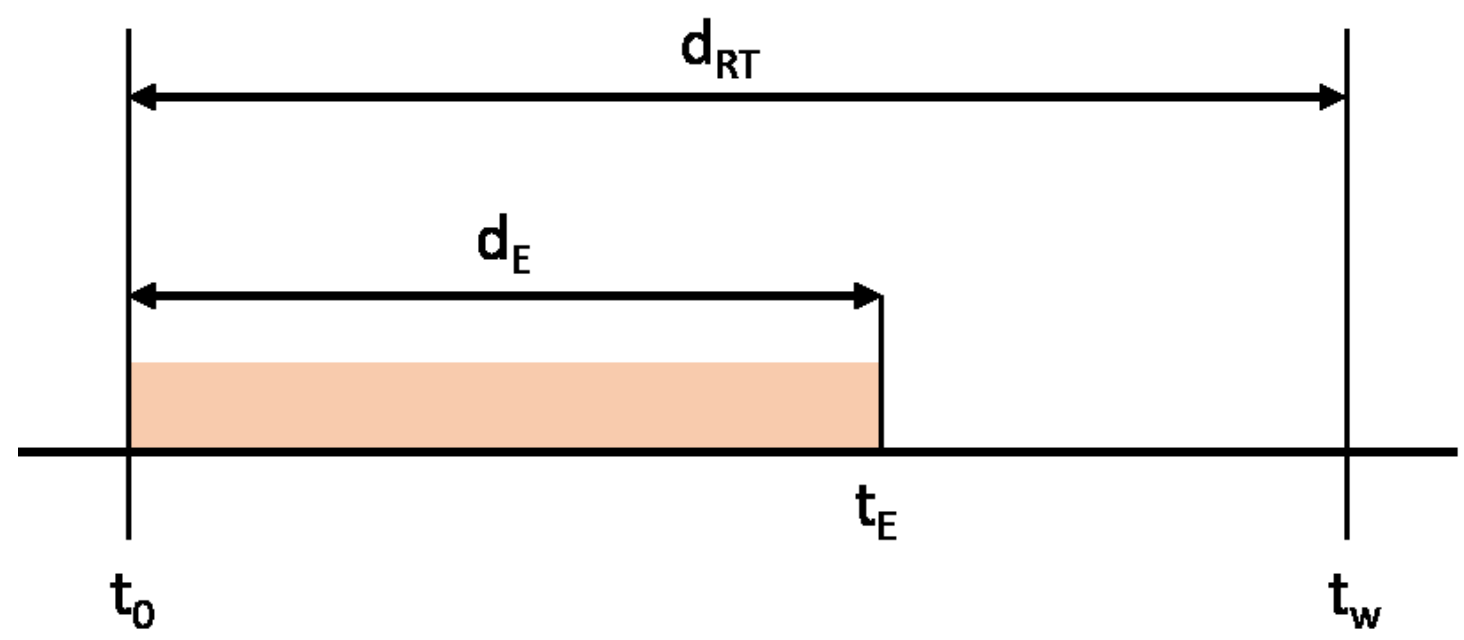

Figure 13: Real-time processing threshold

This figure illustrates processing time versus the real-time processing threshold.

Duration $\mathrm{d}_{\mathrm{RT}}$ (time $\mathrm{t}_{0}$ to $\mathrm{t}_{\mathrm{w}}$ ) is the total time available before the next 'batch' of data is ready to be processed. The end point $\left(t_{\mathrm{w}}\right)$ is the real-time processing threshold.

Duration $\mathrm{d}_{\mathrm{E}}$ is the processing time. If $\mathrm{d}_{\mathrm{E}}>\mathrm{d}_{\mathrm{RT}}$ then the processing of the next batch of data will be delayed by $t_{E}-t_{w}$.

$$
d_{R T}=\frac{l_{W}}{r_{D}}
$$

where $d_{R T}$ is the real-time threshold in seconds, $l_{W}$ is the length of the sample window in samples, and $r_{D}$ is the incoming data rate in samples per second.

This threshold is a hard threshold: if the time taken to process each sample window exceeds this threshold, the system's view of the environment will begin to lag behind the actual condition of the environment (Equation 2).

$$
\operatorname{lag}_{R T}=t_{E}-d_{R T}
$$

where $t_{E}$ is the completion time for each sample window in seconds, and $\operatorname{lag}_{R T}$ is the real-time lag per completion in seconds. 
As this represents the lag time for each sample window processed, the system's view of the environment quickly falls behind (Equation 3).

$$
\operatorname{lag}_{\text {total }}=\sum_{w=1}^{d_{W}} \operatorname{lag}_{R T}(w)
$$

where $d_{W}$ is the duration of the load on the system in windows, and $w$ is the sample window count.

Since sample window lengths differ, this can also be expressed as a normalized fraction of the total sample time (Equation 4).

$$
\operatorname{lag}_{\%}=\frac{t_{E}-d_{R T}}{d_{R T}}
$$

Practically, in most cases a very small latency when generating alarms would likely be acceptable, as it would be an insignificant fraction of the total response time in an emergency. The primary issues with this latency, then, are: first that its duration cannot be determined in advance, especially in general-use devices, and second, that if it is not identified and corrected, over time the system's view of the environment will eventually begin lag by many minutes.

\subsection{Data Analysis}

The smart phone was connected to the local node via a wireless TCP socket connection, and was used to simulate up to five sensors. Each simulated sensor was designed to transmit 16 bit data samples at a frequency of $1 \mathrm{kHz}$. Upon receiving the data, the local node filled a 1000 sample buffer, and then performed a number of iterations of a short-term power calculation. The buffer length was chosen to give a real- 
time processing threshold of 1 second for each simulated sensor. Time to completion data were generated by the local node at the beginning and end of execution, then pushed to the central node via HTTP. These timing data were logged at the central node and later graphed and analyzed using Excel 2013 and Matlab 2014.

\subsubsection{Single Thread}

Figure 14 demonstrates the performance of a single thread operating under a lowload scenario. It is a subset of data that were collected over a number of minutes, and shows the completion time for the operations performed on each 1000 sample window over a sequence of twenty windows. The local node is processing data produced by the smart phone for a single simulated sensor. The red line shows the total time taken to

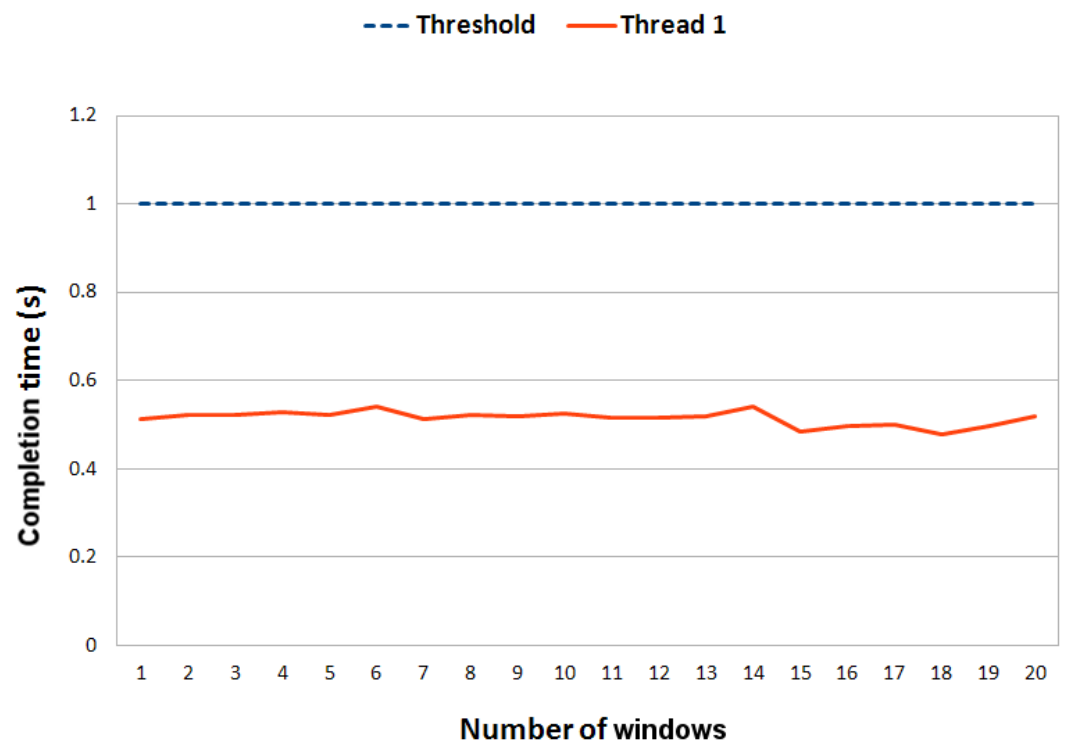

Figure 14: Completion time for data from a single sensor

Completion time of data transmitted over TCP/IP from a single simulated sensor at a rate of $1 \mathrm{kHz}$. Twenty 1000-sample data windows were subject to repeated short-term power calculations and the results compared to the real-time processing threshold. 
perform a number of operations on twenty 1000-sample windows, while the dashed blue line shows the real-time calculation threshold discussed in the previous section. This is the baseline scenario, and demonstrates that the processor is capable of completing each batch of test operations well before reaching the 1 second real time threshold. The small variations in completion time are likely caused by background operations being run by the OS or differences in the state of the device from one sample window to the next.

\subsubsection{Five Sensors of the Same Priority}

Figure 15 demonstrates five threads running simultaneously and processing data from five simulated sensors. Each thread is performing the same set of operations as

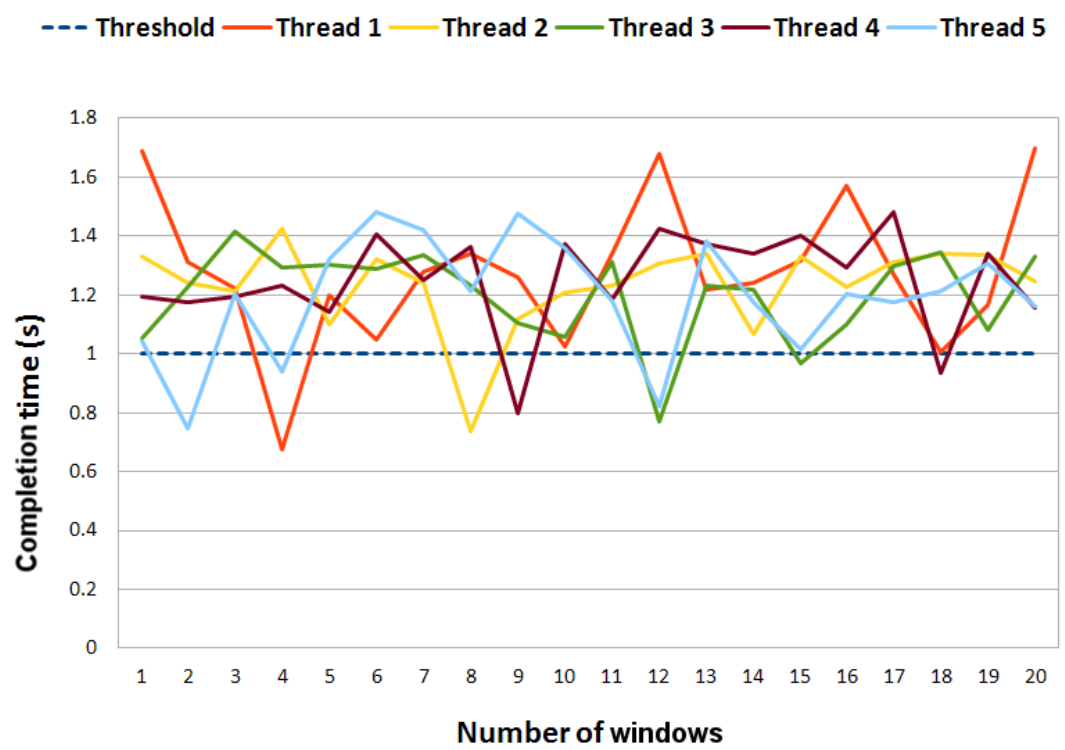

Figure 15: Completion time for data from five sensors being processed at equal priority

Data was transmitted over TCP/IP from five simulated sensors, each transmitting at a rate of $1 \mathrm{kHz}$. For each simulated sensor, twenty 1000-sample data windows were subject to repeated short-term power calculations and the results compared to the realtime processing threshold. 
before, and they are all executing at the same priority. As in Figure 14, the real time threshold is 1 second. Here, no thread is able to perform consistently faster than the real time threshold. Variability in completion times is likely due to OS overhead tasks (which must also be accomplished in the face of heavy processing load), and process management decisions.

\subsubsection{Four Low Priority Sensors and One High}

\section{Priority Sensor}

Figure 16 shows five threads performing the same set of operations with one

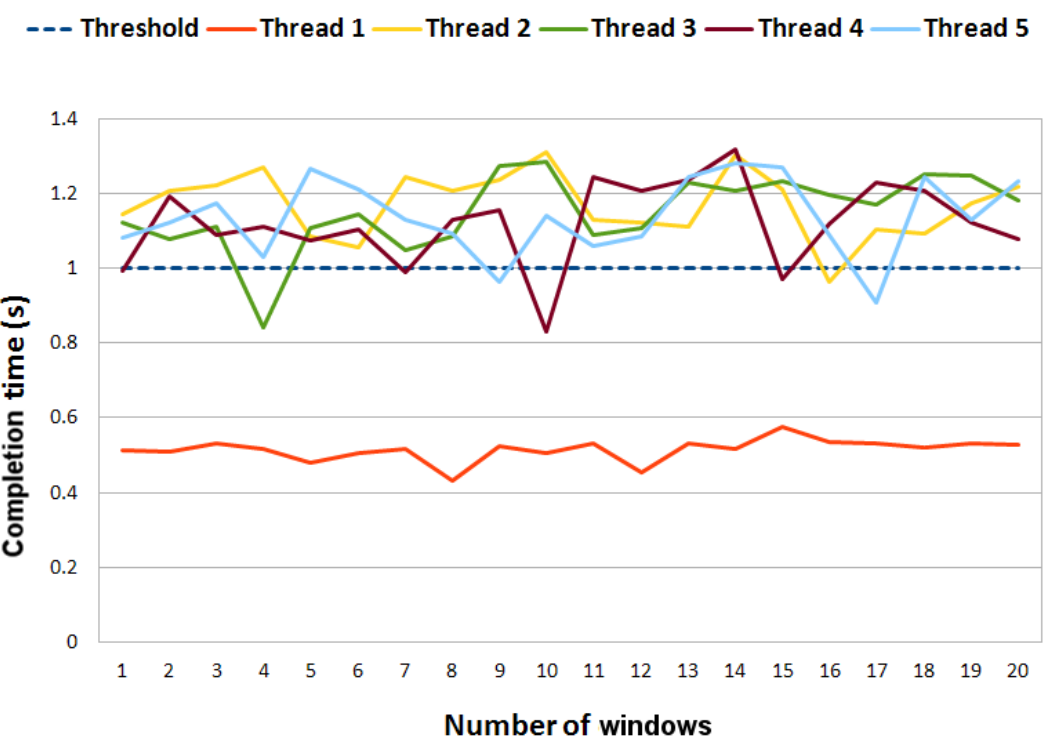

Figure 16: Completion time for data from one high priority sensor and four low priority sensors

Data was transmitted over TCP/IP from five simulated sensors, each transmitting at a rate of $1 \mathrm{kHz}$. For each simulated sensor, twenty 1000 -sample data windows were subject to repeated short-term power calculations and the results compared to the realtime processing threshold. 
assigned a higher priority than the others. The high priority thread (Thread 1) is clearly able to consistently complete execution before reaching the processing threshold, whereas the four remaining threads cannot.

\subsection{Advantages of the RTOS}

In Figure 14, the processor is able to easily keep pace with the incoming data, completing its tasks in roughly half the threshold time. This represents the ideal case, in which the processor is devoted almost entirely to performing a single task. For applications of a type similar to this, there is no particular benefit to using an RTOS. This situation is relatively rare, however, especially for local node applications that take advantage of existing general use devices, such as smart phones, tablets, or personal computers, which may intermittently see heavy user-generated processing load. Figure 15 represents a common scenario when working with such general use devices: even though the processor is ordinarily operating at less than $100 \%$ capacity, certain tasks (or a combination of many) transiently require it to be operating at full capacity. Processes that cannot complete execution within a single time slice (or those that are generated while the system is already doing other work) are forced to wait, and the system must pass control back and forth between each task. This figure represents the operation of a GPOS running a fair scheduling algorithm (such as a round-robin algorithm), in which no single task is given any particular priority. Here, the average completion time for each thread was well above the 1 second hard threshold. The delay introduced by repeatedly missing the real time processing threshold would result in buffering that would cause the system's view of the environment or physiological process being measured to lag behind its actual 
state. Were the system allowed to continue missing the threshold for a significant enough duration, it could result in a lag time of many minutes or even hours. That of course could prove disastrous should an alarm need to be raised in that time. Figure 16 demonstrates the advantage offered by the RTOS. The situation was the same seen in Figure 15: five sensors transmit data simultaneously to the local node, and the local node must perform a certain amount of processing on each. In this case, however, thread 1 was assigned a higher priority than the others. The high priority thread received a larger percentage of the available clock cycles than did the low priority ones, so its average completion time was nearly unaffected by the other threads running alongside it. This represents a scenario in which several tasks need to be performed simultaneously, but only one is time-critical in nature. This kind of operation is impossible with a GPOS that uses a fair scheduling algorithm. The RTOS, on the other hand, can choose particular sensors to process at a high priority, or can dynamically assign lower-priority tasks to be completed at a higher priority should they begin to approach their timing threshold.

The RTOS used in this system is the QNX Neutrino microkernel RTOS. It was selected primarily for two reasons: first, it is the OS used by many car manufacturers; and second, it is highly scalable and is compatible with a variety of microcontrollers that can be installed as relatively inexpensive dedicated local nodes in the home environment. It was designed for use in computing systems of any scale, but with a particular focus on embedded systems. One of the key design considerations in an embedded system is processing power. Many embedded systems are battery powered, or designed to be produced in large quantities, and they are therefore designed to use the slowest (and therefore lowest cost) processing units possible for the application. The QNX Neutrino 
operating system utilizes a modular "microkernel" design that reduces OS processing overhead and gives the developer the ability to include only the OS components required by the application (the OS was left in its default state for development purposes, but extraneous components, such as the display could be removed if the device were to be made commercially available). This flexibility offers significant advantages in developing on a low cost device. Since, for installation in the home environment, the local node of the implemented system is designed to be installed on-site with the sensors, low cost per unit is of interest.

\subsection{Conclusion}

This chapter presented an argument for the importance of accounting for the hard processing threshold involved in real-time processing, and demonstrated that, in devices operating under heavy processing load, an RTOS is superior to a GPOS and offers more options for maintaining a real-time link with critically important sensors. While this capability may not be required for every RPM application, as discussed in this chapter, it is a critical design parameter in those applications that do require it. It must, then be included in this system in order for it to be adaptable to a variety of monitoring tasks. Specifically, if the system is installed in a home or hospital environment, the ability to generate real-time alarms will be a key design parameter. 


\section{Chapter 6:}

\section{Adaptive Accelerometer Calibration}

\subsection{Introduction}

The RPM system developed and tested in the preceding chapters can be deployed in many environments and with many types and configurations of sensing equipment. One important, and sometimes overlooked, environment is the personal vehicle. For older adults, the ability to drive is an important factor for maintaining independence, but for those experiencing functional impairment or cognitive decline, driving eventually becomes unsafe for them and for the public. It is, therefore, important to have a clear view of an individual's capacity to drive so that informed decisions regarding driving cessation can be made.

It should be clear to anyone who has been a passenger in a car that driving habits differ from person to person. Some drive very smoothly, while others accelerate and brake abruptly. Still others will 'pump' the brake, leading to a bouncing sensation in the car, or they may habitually take turns too quickly. An illustration of these acceleration waveforms is shown in Figure 17, and from these it seems likely that a distinction between drivers could be made. It has therefore been hypothesized that an individual's 


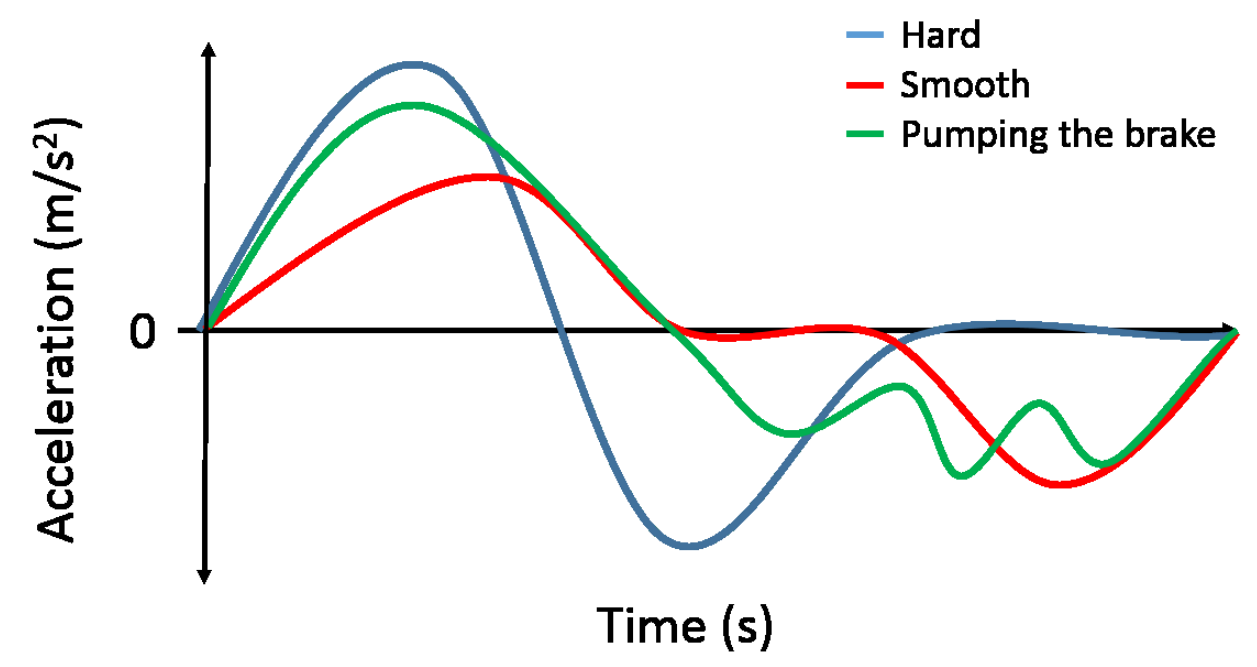

Figure 17: Illustration of different acceleration patterns

An illustration of three common driver acceleration patterns. The blue line represents those who habitually accelerate and brake abruptly, while the red line represents a smoother, slower approach. The green line is a method commonly used to brake in winter driving conditions (though there are some who drive this way habitually), and features 'pumping' the brake to stop while still maintaining control of the vehicle.

patterns of driving behavior may be characteristic, and that some patterns may be associated with cognitive or physical decline and increased collision risk. Additionally, because driving is a very high-order human function (i.e. it requires the coordinated use of a number of abilities such as concentration, physical coordination, memory, etc...), and the failure of the high-order functions herald overall body system failure, declining ability to drive may be an early sign of worsening medical problems. This chapter covers the early stages of exploration into these ideas by investigating patterns of acceleration and deceleration using an accelerometer and the Candrive sensor described in Chapter 4 . Specifically, this chapter describes an accelerometer calibration method that combines a direct measure of acceleration with a reference velocity measure. It opens by describing the techniques and sensors used in the data collection. It next describes sources of error in 
accelerometer measurements and the challenges associated with calibrating accelerometer data without a reference velocity. It then considers ways in which several research groups have tried to tackle the issue. This chapter also lists a few of the failed, exploratory attempts at calibrating the data, and then details the method that was eventually decided upon and reports the results. Finally, it describes the ways in which the corrected acceleration signals can be used in monitoring driver risk, and speculates on some of the benefits of using this adaptive filtering technique on accelerometer measurements in general.

\subsection{Data Collection}

A total of 16 acceleration profiles were collected in which the vehicle accelerated from stopped to a maximum speed of between 50 and 80 kilometers per hour, and then returned to a full stop. The three smart phones were deployed in different orientations within the vehicle as summarized below:

- All three phones with top in the direction of travel.

- Two phones with bottom in the direction of travel and one phone with top in the direction of travel.

- All three phones with left side in the direction of travel.

- Two phones oriented with right side in the direction of travel and 1 phone with left side in the direction of travel.

For each of the above four configurations, four acceleration profiles were recorded on each of the three smart phones. This combination thereby provides three sets of 16 acceleration measurements. 


\subsection{Calibration Challenges}

Acquiring accurate measurements from accelerometer data presents a number of challenges. Firstly, all accelerometers are very sensitive to vibration noise, as shown in Figure 18. Secondly, they are often susceptible to baseline drift, as shown in Figure 19. Finally, they are prone to inaccuracies in all aspects of factory calibration including offset (Figure 20), gain (Figure 21), and orthogonality (Figure 23) (this is especially true of the IMUs installed in modern smart phones, which are designed to be low cost). In addition to these physical limitations, in order to translate acceleration data into position or

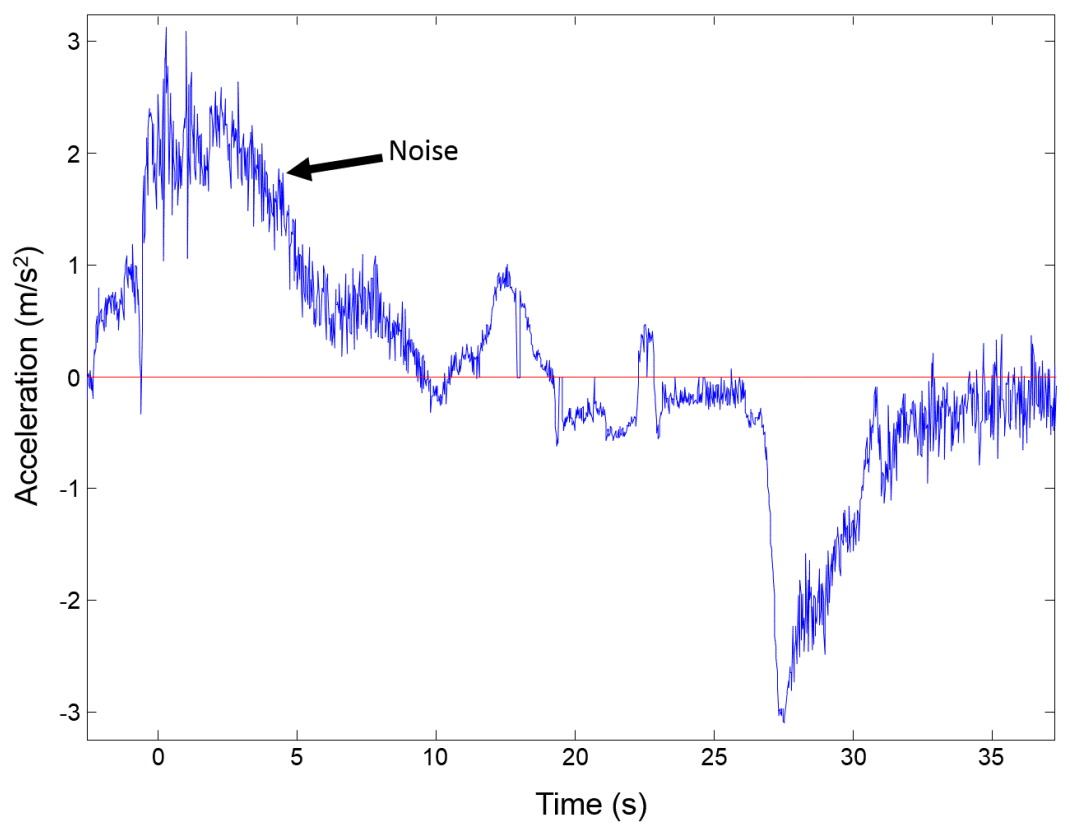

Figure 18: Effect of vibration noise on the accelerometer waveform

These data were obtained by a MEMS accelerometer built into a smart phone as part of the two hours of driving data collected for the accelerometer calibration experiment. The figure shows the effect of vibration noise over fifteen seconds during which the vehicle remained stationary. 
velocity data, it is necessary to integrate. This means any noise or drift will result in significant cumulative error problems over time, as shown in Figure 22. This drift is especially problematic, because, as shown in Figure 24, the majority of useful accelerometer signal power is also very low frequency, rendering straightforward filtering difficult.

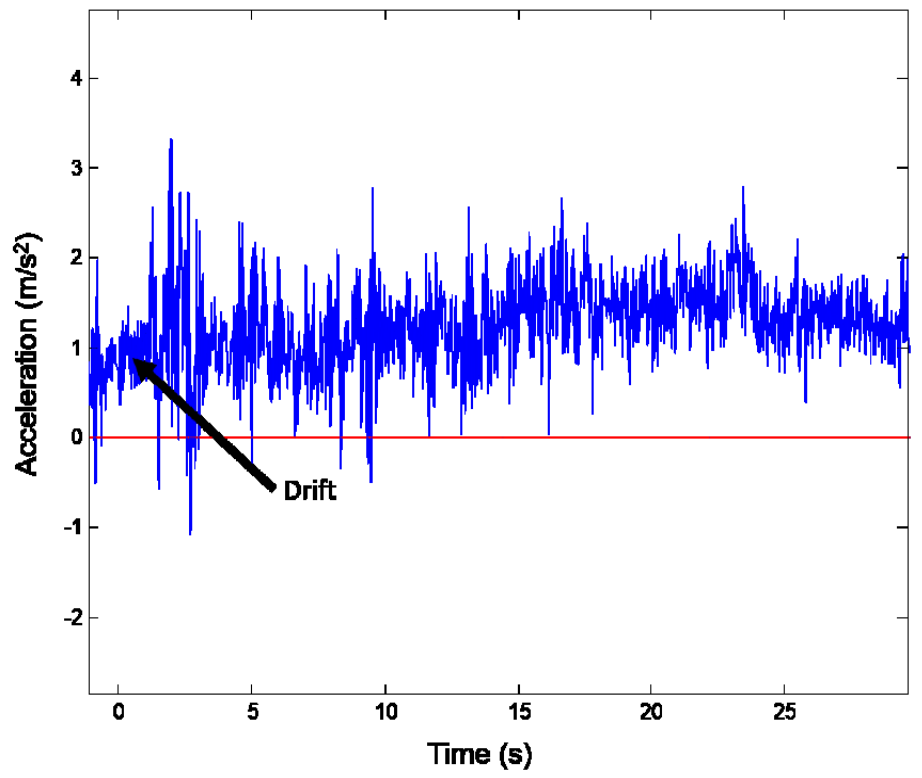

Figure 19: Effects of baseline drift on the acceleration waveform

These data were obtained by a MEMS accelerometer installed in a smart phone as part of the two hours of driving data collected for the accelerometer calibration experiment. At 90 minutes, the baseline acceleration drifts over a duration of approximately one minute from $-0.5 \mathrm{~m} / \mathrm{s}^{2}$ to greater than $1 \mathrm{~m} / \mathrm{s}^{2}$. 


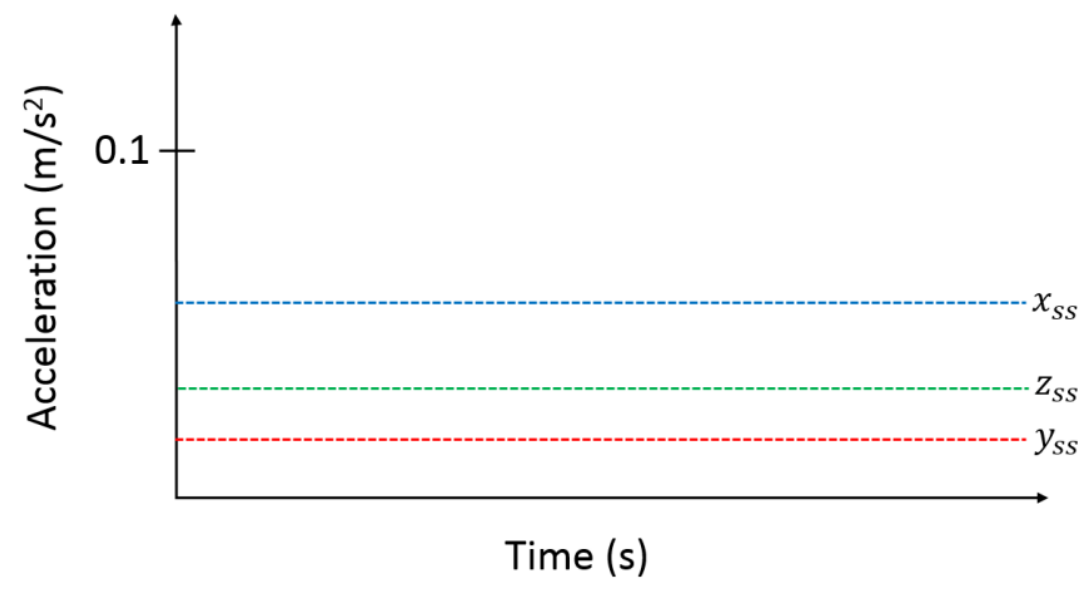

Figure 20: Illustration of accelerometer offset error

Accelerometer offset calibration is often imprecise, so that when the device is sitting still, it does not read exactly zero, but rather some small, constant value greater than zero. This contributes significantly to velocity and position drift: if the offset is even as low as $0.01 \mathrm{~m} / \mathrm{s}^{2}$, the measured velocity will be off by $0.6 \mathrm{~m} / \mathrm{s}$ after one minute.

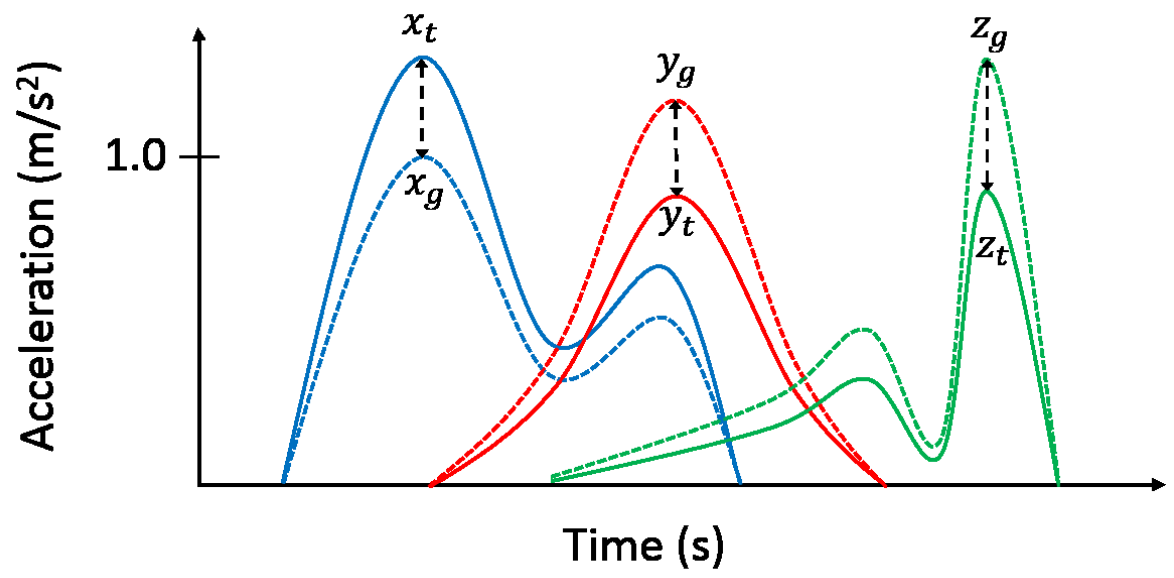

Figure 21: Illustration of accelerometer gain error

Imprecision in manufacturing and calibration can also lead to acceleration gain errors. This can result in under- or over-shooting the true value of the acceleration. In this illustration, $\mathrm{x}_{\mathrm{g}}, \mathrm{y}_{\mathrm{g}}$, and $\mathrm{z}_{\mathrm{g}}$ represent the measured value of the acceleration and $\mathrm{x}_{\mathrm{t}}, \mathrm{y}_{\mathrm{t}}$, and $\mathrm{z}_{\mathrm{t}}$ represent the true value of the acceleration. 


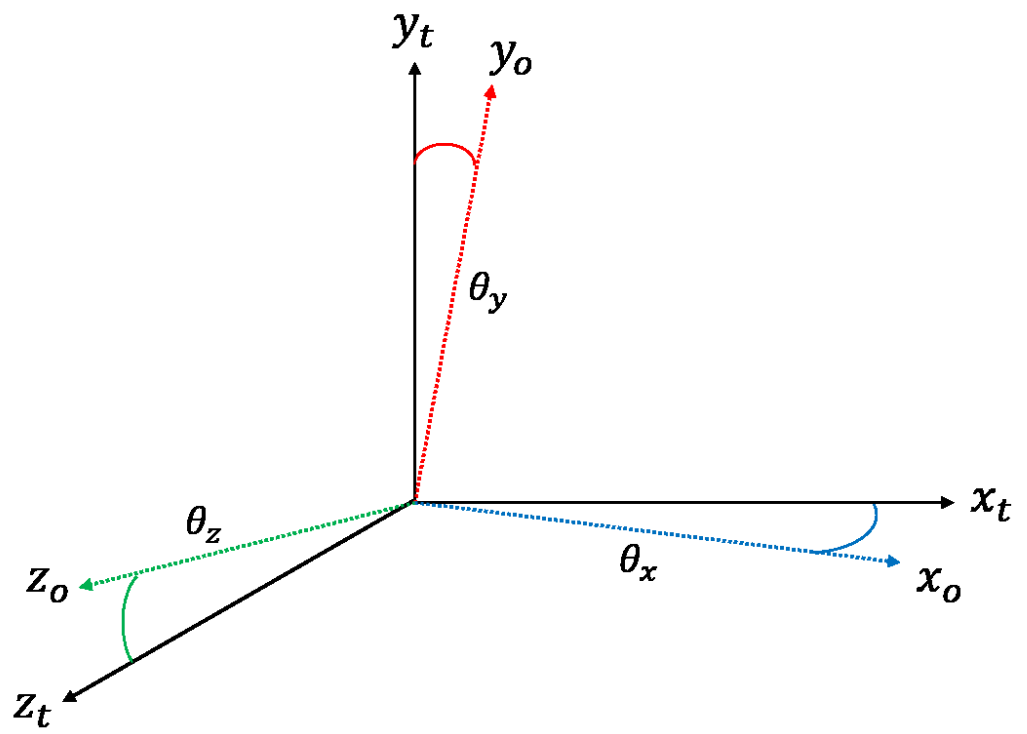

Figure 23: Illustration of accelerometer orthogonality error

The axes that accelerometers measure along are not necessarily precisely orthogonal to each other or to the orientation of the board they are printed on. This can lead to problems using the accelerometer to measure orientation, and can contribute to both of the other sources of error.

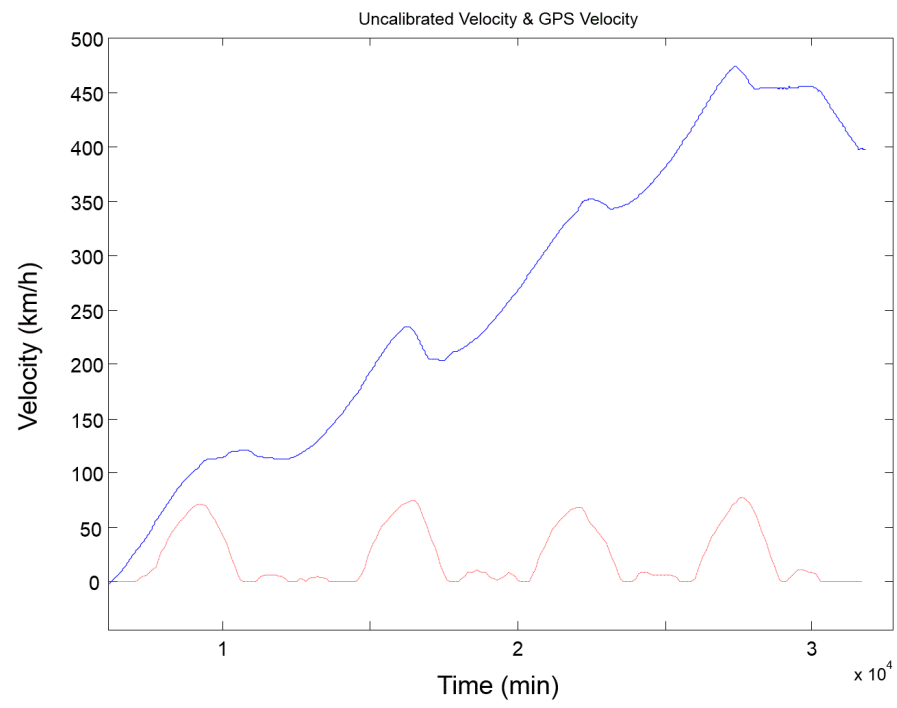

Figure 22: Integration error over six minutes of driving data.

This chart shows the OBDII velocity in $\mathrm{km} / \mathrm{h}$ in red, and the velocity calculated from the raw acceleration data in blue. It illustrates how quickly integration error can accumulate. Even after only six minutes of driving, the true signal is almost completely eradicated. These data were collected as part of the two hours of driving data collected for the accelerometer calibration experiment. 


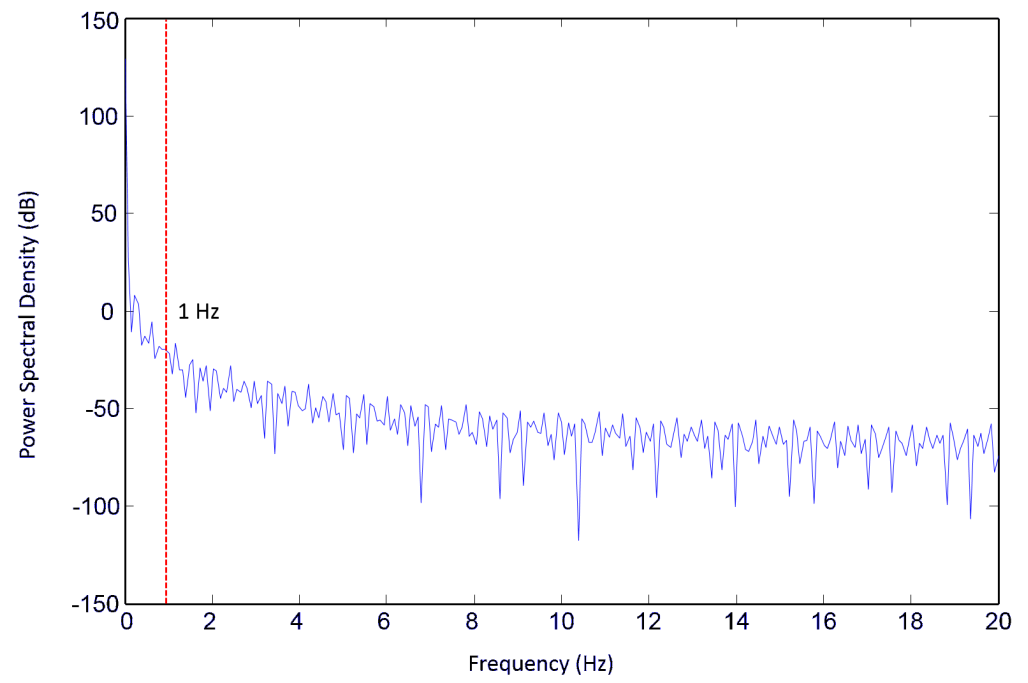

Figure 24: Power Spectral Density of the acceleration signal.

The majority of the signal power is concentrated at or near $0 \mathrm{~Hz}$. Any attempt at a simple high-pass filter to remove the baseline drift effect also destroys a significant portion of the useful information.

A number of methods for resolving the many problems associated with accelerometer calibration have been proposed. Some have worked on improving accelerometer manufacturing techniques and materials, and have proposed a polymerbased fabrication technique for a three axis accelerometer [98], while others have investigated post-manufacturing methods for improving the calibration of existing devices.

\subsection{Explorations}

During the course of the work for this thesis, several solutions were attempted to the problems which were not successful. A number of them are summarized in Table 3. Most other accelerometer calibration methods in the literature require the use of 
specialized equipment for precisely orienting the accelerometer relative to the gravity vector, so initially there was a period of exploration of automatic calibration methods. The goals were to programmatically eliminate noise and correct for each source of factory error (offset, gain, orthogonality) in order to correct the signal.

Table 3: Initial attempts to calibrate the data using a variety of methods. These were largely probing in nature, as an attempt to get an idea of the shape of the data.

\begin{tabular}{|l|l|l|}
\hline \multicolumn{1}{|c|}{$\begin{array}{c}\text { Exploratory } \\
\text { Solution }\end{array}$} & \multicolumn{1}{c|}{ Result } & \multicolumn{1}{c|}{ Comment } \\
\hline $\begin{array}{l}\text { Empirically identify } \\
\text { offset and remove it }\end{array}$ & $\begin{array}{l}\text { Minor } \\
\text { improvement in } \\
\text { calculated velocity }\end{array}$ & $\begin{array}{l}\text { There does seem to be some } \\
\text { consistent offset, but that } \\
\text { offset appears to drift } \\
\text { (sometimes quite drastically). }\end{array}$ \\
\hline $\begin{array}{l}\text { High-pass filter the } \\
\text { acceleration signal } \\
\text { significant } \\
\text { distortion of the } \\
\text { output acceleration } \\
\text { signal }\end{array}$ & $\begin{array}{l}\text { There is a lot of useful low- } \\
\text { frequency information that is } \\
\text { attenuated along with the } \\
\text { baseline offset value. }\end{array}$ \\
\hline $\begin{array}{l}\text { Scale the acceleration the calculated } \\
\text { jerk (the derivative of } \\
\text { acceleration) }\end{array}$ & $\begin{array}{l}\text { Improvement of } \\
\text { calculated } \\
\text { velocity, distortion } \\
\text { of acceleration } \\
\text { maxima and } \\
\text { minima }\end{array}$ & $\begin{array}{l}\text { The jerk value approaches 0 } \\
\text { when steady-state is reached, } \\
\text { but also at acceleration } \\
\text { maxima and minima. Search } \\
\text { area can be widened to } \\
\text { prevent misidentification of } \\
\text { maxima/minima as steady- } \\
\text { state, but at the cost of failing } \\
\text { to correct short regions of no } \\
\text { acceleration. }\end{array}$ \\
\hline $\begin{array}{l}\text { Correct the velocity, } \\
\text { position, and } \\
\text { acceleration signals } \\
\text { using a Kalman filter }\end{array}$ & Very good output & $\begin{array}{l}\text { Doesn't actually correct the } \\
\text { accelerometer signal. }\end{array}$ \\
\hline
\end{tabular}

Initially, attempts were made to automatically identify and remove factory offset and gain errors. This was accomplished through the application of moving averages and programmatic empirical identification. This proved problematic as the accelerometer offset was found to drift, and the correction algorithms were unable to completely 
eliminate the integration error issues. Following this, several attempts were made to highpass filter the acceleration signal to remove the drift, but this destroyed much of the important low frequency information and resulted in unacceptable signal distortion (Figure 25).

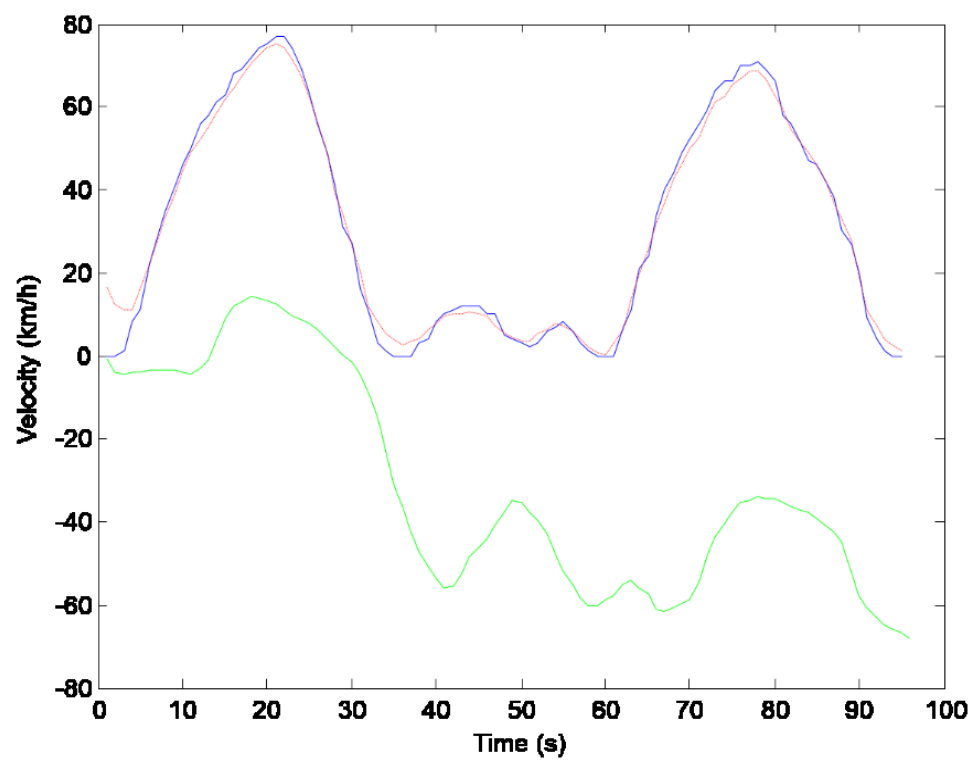

Figure 25: Effect of a high pass filter on the acceleration signal

True velocity is shown in red, and high pass filtered accelerometer calculated velocity is shown in blue. While the filter did largely eliminate the drift effect seen in Figure 22 , the velocity signal was significantly distorted in the process.

An attempt was also made to utilize the derivative of the acceleration (known as the 'jerk') to identify regions of steady state in the acceleration signal (which indicate periods where the vehicle may be stopped) and eliminate the offset. This technique did reduce the effect of integration error, but it was also found to attenuate maxima and minima in the acceleration signal. Additionally, this method would fail during periods in which the vehicle was traveling at a constant speed, and so would have only limited 
utility without some other measure of the vehicle's speed.

The last attempted solution, and the most promising, was to employ a Kalman filter to obtain a corrected signal. This technique was able to achieve a very good correction through making use of all three main measures of movement: GPS position, OBDII velocity, and accelerometer acceleration (Figure 26). While promising, this technique was not further investigated primarily because it merely adjusts its weighting to compensate for erratic sensor behavior, it does not actually correct the sensor output. In consequence, instead of a corrected signal, the quality of the output of this filter can be less consistent, as the filter tunes out data that are perceived to be incorrect.

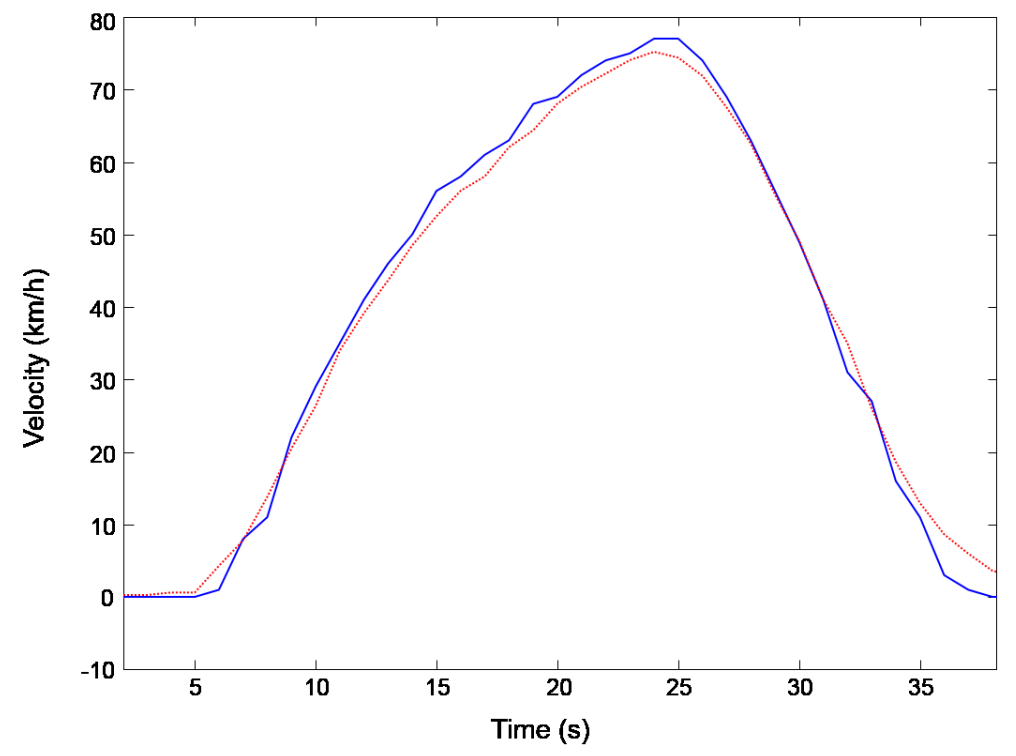

Figure 26: Kalman filter performance

The result of employing a Kalman filter with GPS position, OBDII velocity, and accelerometer acceleration. Reference velocity is shown in red, Kalman filter output velocity shown in blue. The Kalman filter produces an accurate output, but it does so by adjusting for inaccurate sensor behavior and does not not actually correct the acceleration waveform directly. 


\subsection{Adaptive Filtering Technique}

Accelerometer use over longer periods of time is impacted by offsets and by drift in the accelerometer readings that may be caused or exacerbated by continuous excitation from vibration noise [50]. This is shown in Equation 5, where the actual accelerometer value has Gaussian noise $\left(Z_{a}\right)$ and non-stationary drift component $(d)$.

$$
A_{m}[m]=A_{a}[m]+Z_{a}[m]+d[m]
$$

The GPS and OBDII sensors provide two different measures of vehicle velocity and, as shown in Equation 6, the actual velocity measure is assumed to contain noise $\left(Z_{v}\right)$.

$$
V_{m}[n]=V_{a}[n]+Z_{v}[n]
$$

In a system without noise or drift, the higher sampling rate acceleration signal $\left(f_{S P}\right.$ $=40 \mathrm{~Hz})$ can be integrated as shown in Equation 7 to provide the actual velocity at sampling rate $\left(f_{C D}=1 \mathrm{~Hz}\right)$ where the noise signal $\left(Z_{I}\right)$ is introduced because of error associated with discrete signals. The decimation ratio $(\mathrm{R})$ is provided by Equation 8 .

$$
\begin{gathered}
V_{a}[n]=V_{a}[n-1]+\sum_{\mathrm{m}=\mathrm{Rn}-(\mathrm{R}-1)}^{\mathrm{Rn}} A_{a}[m] / f_{S P}+Z_{I}[n] \\
\mathrm{R}={ }^{f_{S P}} / f_{C D}
\end{gathered}
$$

Even so, for a system with drift and noise, integrating each of the sets of $\mathrm{R}$ acceleration samples will introduce an error in the predicted velocity. The OBDII and GPS sensors provide an independent measure of velocity at a lower sampling rate that can be used as a reference signal to adaptively correct the accelerometer readings. The correction enables the use of the higher sampling rate accelerometer, which can give a 
more detailed view of the acceleration waveform and more information from which to eventually derive a driver signature.

The method for adaptive correction of the accelerometer signals begins with Equation 9, in which the predicted value for the velocity is calculated based on the previous measured reference velocity and the integration of the acceleration signals $\left(A_{c}\right)$ that include all previous calculated corrections, accounting for the higher sampling rate of the smart phone signals (the algorithm waits to have at least one set of measurements from each sensor before it begins). From this, an error signal $(\epsilon)$ can be determined through the difference of the predicted velocity and the measured velocity from either the GPS or OBDII sensor, shown in Equation 10.

$$
\begin{gathered}
V_{p}[n]=V_{m}[n-1]+\sum_{\mathrm{m}=\mathrm{Rn}-(\mathrm{R}-1)}^{\mathrm{Rn}} A_{c_{n-1}}[m] / f_{S P} \\
\epsilon_{\mathrm{n}}=V_{m}[n]-V_{p}[n]
\end{gathered}
$$

This error signal can then be used to iteratively update the correction to the acceleration signal through Equation 11, where the current and future acceleration values are updated based on a delta update function. Two different delta functions are analyzed as shown in Equation 12 and 13. In Equation 12, the update for the current window of $\mathrm{R}$ samples is flat, while in Equation 13, the update is a ramp function (the scaling coefficient is a function chosen so that the triangular shape formed by the ramp would have unity area over R samples). The step delta is an intuitive choice, but since the error being corrected included a drift component, the ramp shape was also investigated because it allows for the correction to grow across the window. In both cases, the delta function 
is scaled so that it provides $100 \%$ correction while accounting for the over sampling of the acceleration signals.

$$
\begin{gathered}
A_{c_{n}}[m]=A_{c_{n-1}}[m]+\Delta[\mathrm{m}] * \epsilon_{\mathrm{n}} ; m \geq R(n-1)+1 \\
\Delta_{\text {step }}[\mathrm{k}]=\mathrm{f}_{\mathrm{SP}} \\
\Delta_{\mathrm{ramp}}[\mathrm{k}]=\mathrm{k} * \mathrm{f}_{\mathrm{SP}} *(1 /(\mathrm{R}+1)) ; \mathrm{k}=1: \mathrm{R} \\
=\mathrm{R} * \mathrm{f}_{\mathrm{SP}} *(1 /(\mathrm{R}+1)) ; \mathrm{k}>R
\end{gathered}
$$

The correction algorithm is summarized in Figure 27. Although it only corrects accelerometer data taken from a single axis of motion in this case, the algorithm could be easily applied to all three axes of motion or to a vectorized acceleration.

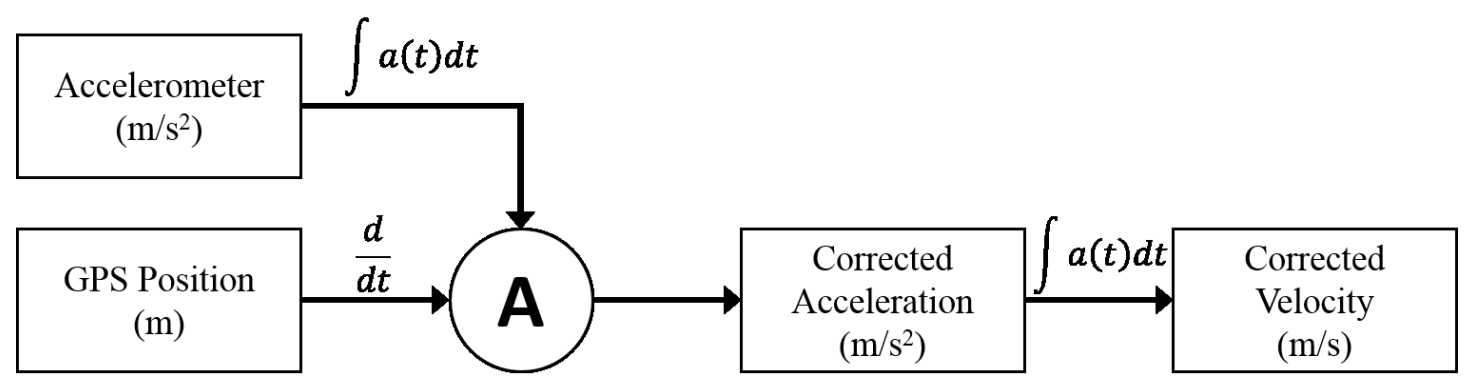

Figure 27: Adaptive correction algorithm

The high sampling rate accelerometer measurements are integrated and fed into the correction algorithm along with the derivative of the low sampling rate GPS position. The algorithm outputs an acceleration at the sampling rate of the accelerometer that has been corrected based on the GPS calculated velocity. This can then be integrated to provide a corrected measure of the velocity or the position. 


\subsection{Performance Measurement}

The performance of the adaptive algorithm can be measured through three performance measures:

- $\quad$ Error signal average power

- $\quad$ Stability of resulting calibrated acceleration and velocity signals.

- $\quad$ Correlation of the resulting acceleration signals.

The correlation of three accelerometer signals for each profile is measured using Equations 14 and 15 where $\mathrm{h}[\mathrm{m}]$ and $\mathrm{k}[\mathrm{m}]$ are the two acceleration profiles being compared.

$$
R=\left(\frac{\sum_{m} h^{*}[m] k[m]}{\sqrt{E_{h} E_{k}}}\right)
$$

Where:

$$
E_{h}=\sum_{m}(h[m])^{2}
$$




\subsection{Accelerometer Calibration}

Figure 28 illustrates the difference between velocity profiles measured using the OBDII velocity sensor and the GPS position based velocity sensor. The upper plot shows the two measures of the profile in the time domain, while the lower plot shows the power spectrum for each measure. The similarity between the profiles is clear, but it is also clear in both the upper and lower plots that the OBDII signal contains a small amount of high frequency information or noise that is missing from the GPS calculated velocity.
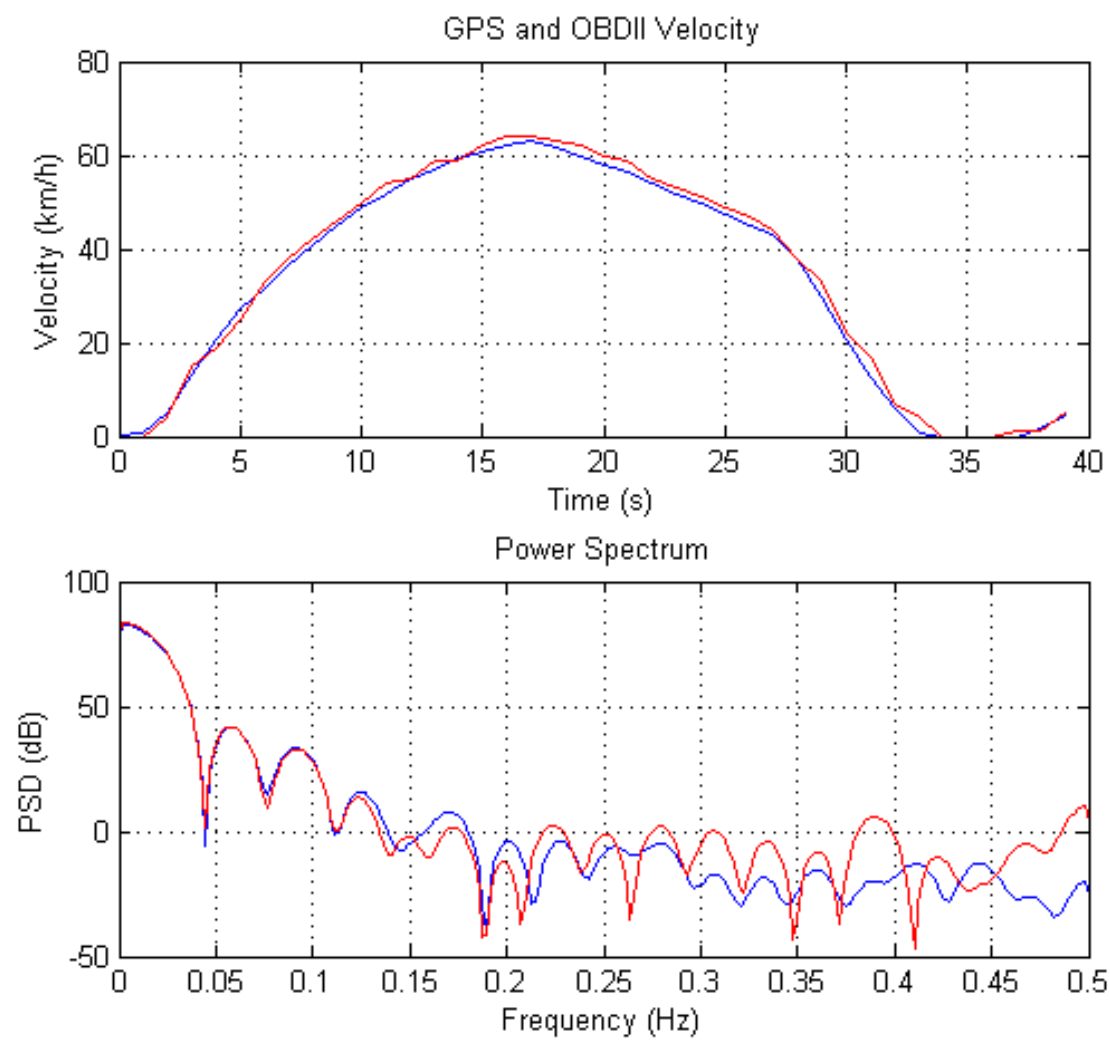

\section{Figure 28: Example acceleration/deceleration profile in time and frequency domain.}

The GPS sensor results are shown in blue and the OBDII sensor results are shown in red. The upper plot shows the time domain for the profile while the lower plot shows the frequency domain. 
Figure 29 shows a comparison between the achievable accelerometer correction and the uncorrected signal. Even within a 40 second timeframe, the effect of the drift is visible, as the resulting acceleration profile is significantly affected and instead of returning to zero velocity, the car has a negative velocity.
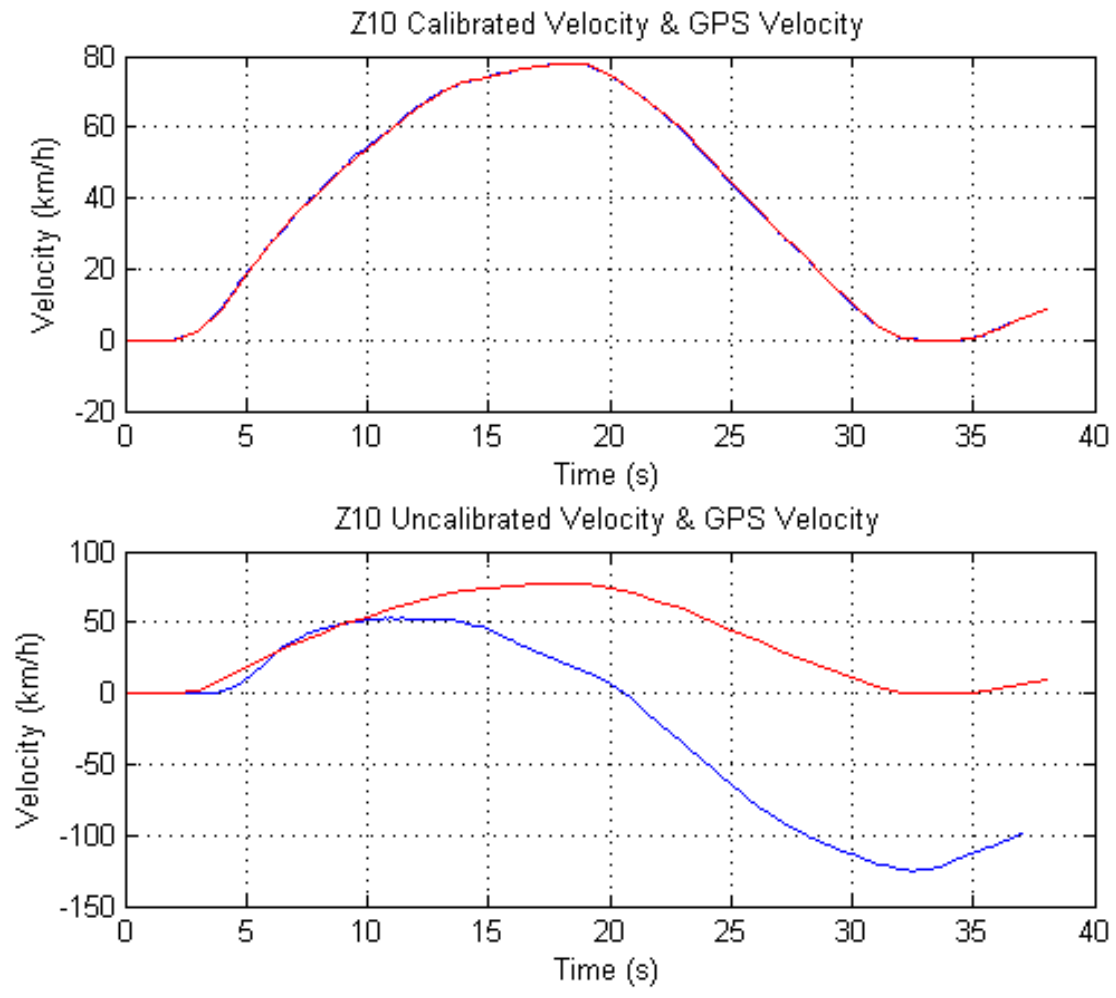

Figure 29: Accelerometer calculated velocity before and after calibration

Accelerometer calculated velocity is shown in blue, and the reference GPS velocity is shown in red. The velocity correction has achieved near $100 \%$ accuracy, while the uncorrected calculated velocity strays very quickly. 
Figure 30 shows the effect of using the ramp shaped delta function in the correction algorithm. The higher end point of the function results in overcorrection and oscillations, as well as a sharp increase in the measured error.
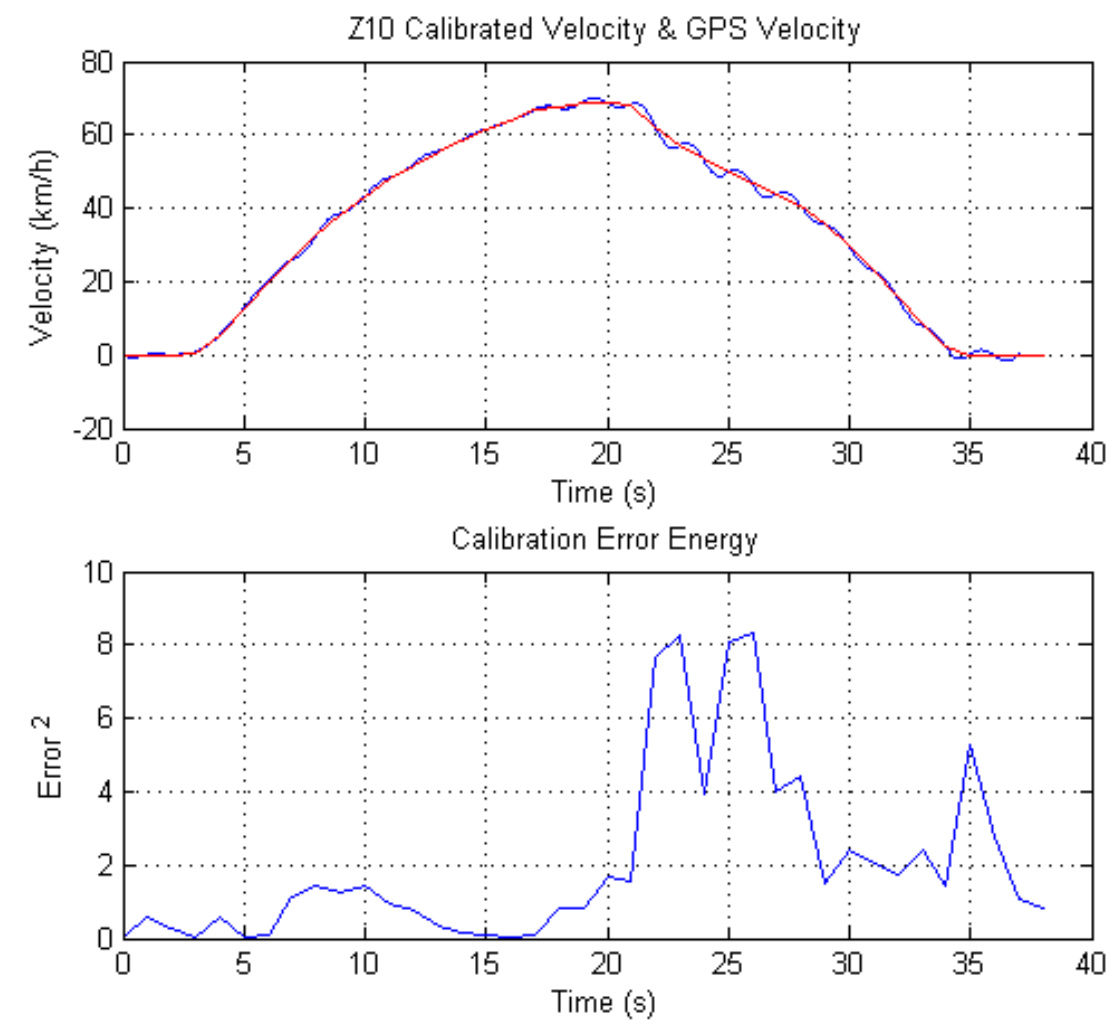

Figure 30: Acceleration profile using a ramp-shaped delta function compared to GPS velocity.

The upper plot shows an example acceleration/deceleration profile using the ramp shaped delta function (shown in blue) compared to the reference GPS velocity measure (shown in red). The lower plot shows the square of the error calculated by the algorithm. 
Figure 31 shows the effect of the ramp delta function to correct the accelerometer using the OBDII as a reference velocity. Here, the high frequency components of the OBDII measured velocity lead to significant oscillation in the resulting error signal. In both cases, the ramp function leads to an over correction of the subsequent acceleration values, leading to unacceptable oscillation in the corrected velocity signals.
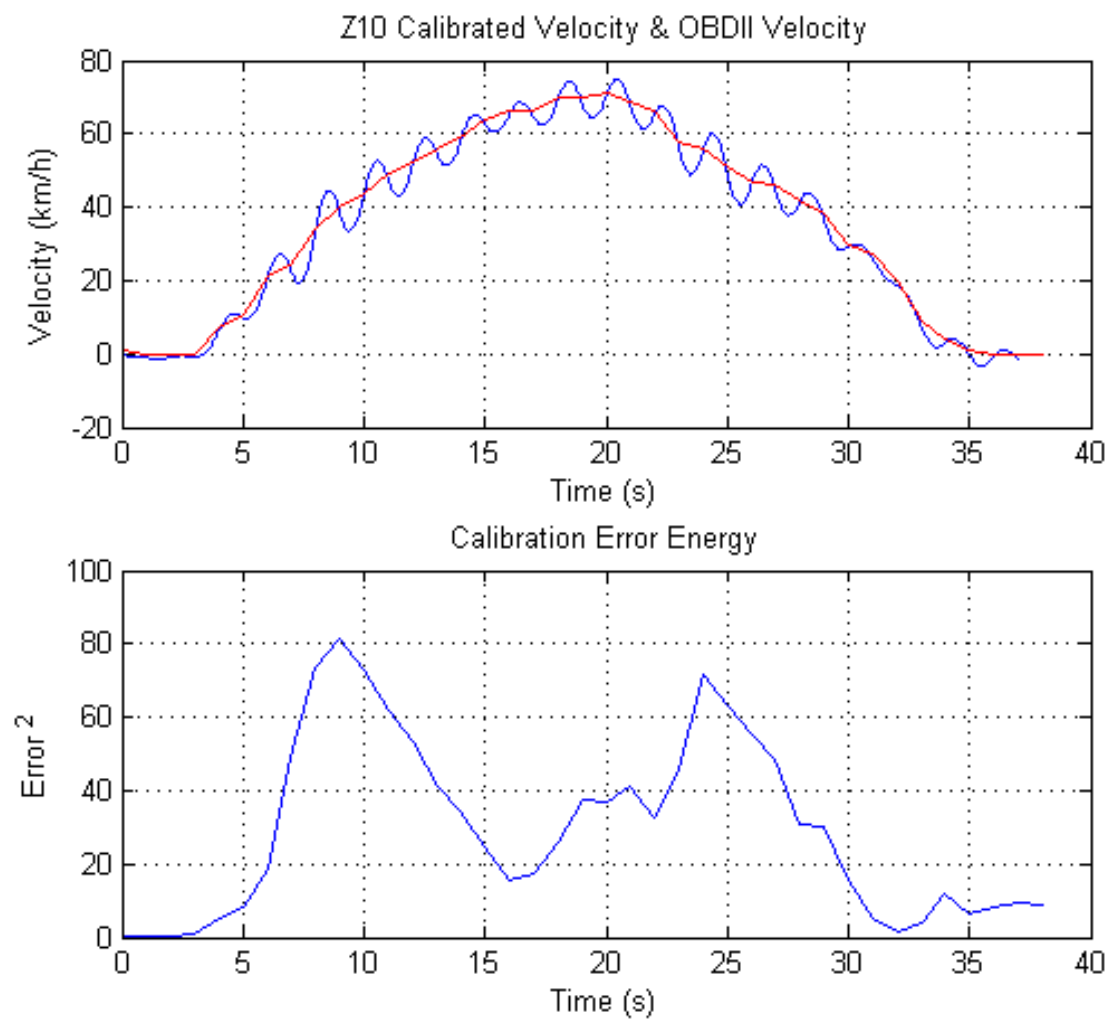

\section{Figure 31: Acceleration profile using a ramp-shaped delta function compared to OBDII velocity.}

The upper plot shows an example acceleration/deceleration profile using the rampshaped delta function (shown in blue) compared to the reference OBDII velocity measure (shown in red). The lower plot shows the square of the error calculated by the algorithm. 
Figure 32 shows the step shaped delta function applied to the correction algorithm, using the GPS derived velocity as the reference. This delta function improves the performance of the correction algorithm considerably, nearly eliminating the oscillation effect.
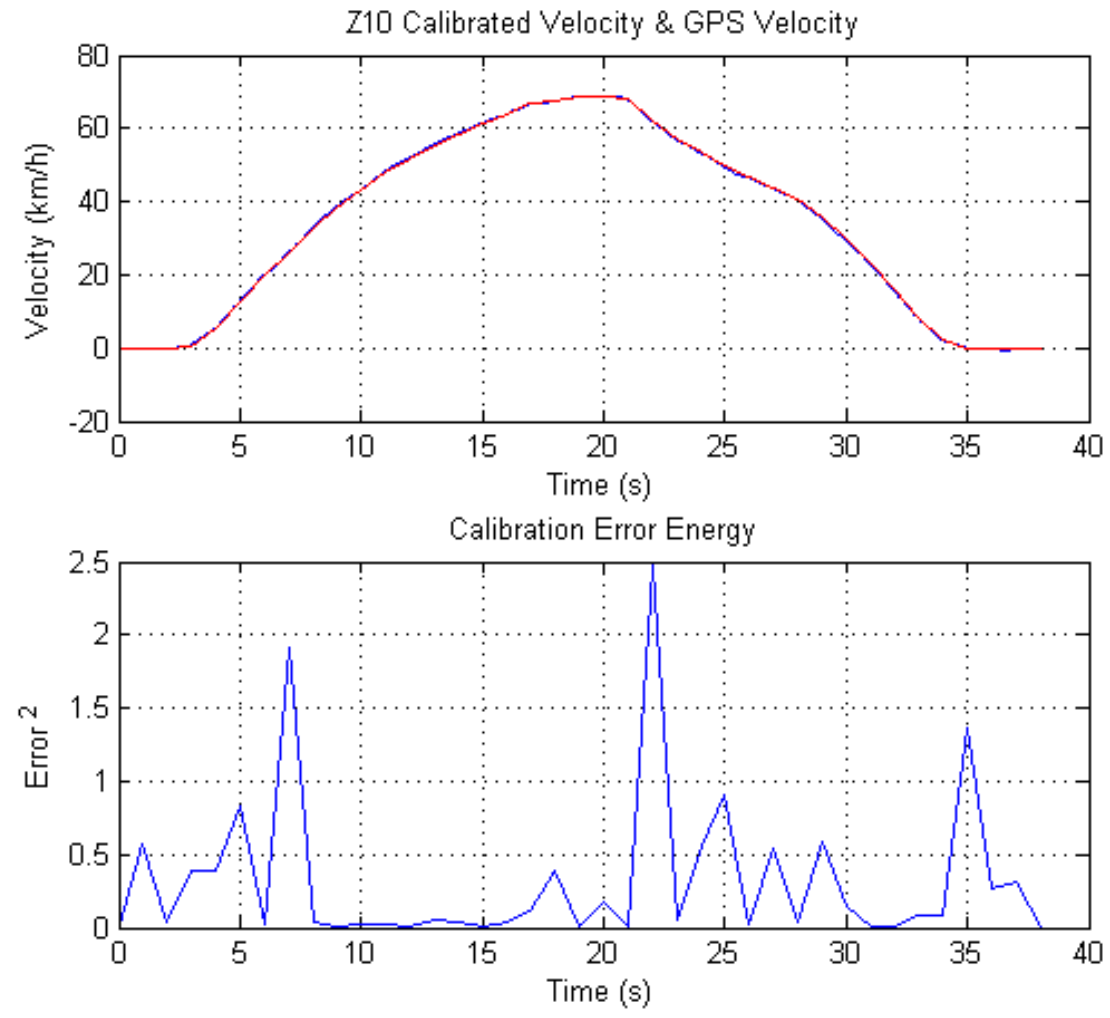

Figure 32: Acceleration profile using a step-shaped delta function compared to GPS velocity.

The upper plot shows an example acceleration/deceleration profile using the stepshaped delta function (shown in blue) compared to the reference GPS velocity measure (shown in red). The lower plot shows the square of the error calculated by the algorithm 
Figure 33 shows the correction algorithm using same step delta function as above, but with the OBDII measured velocity as a reference. Here, it is once again possible to see the oscillation effect from the higher frequency component of the reference velocity measure.
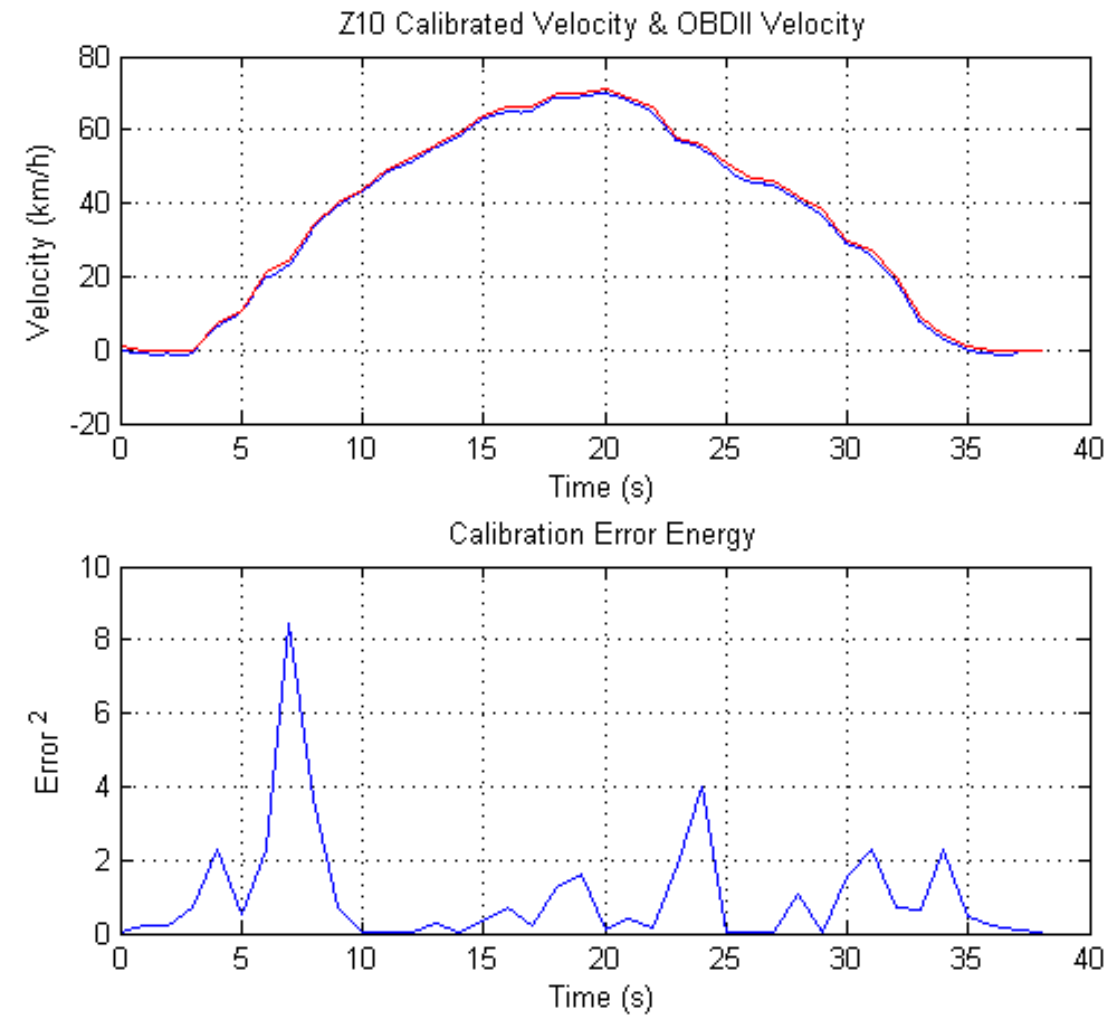

Figure 33: Acceleration profile using a step-shaped delta function compared to OBDII velocity.

The upper plot shows an example acceleration/deceleration profile using the stepshaped delta function (shown in blue) compared to the reference OBDII velocity measure (shown in red). The lower plot shows the square of the error calculated by the algorithm 


\subsubsection{Error Signal Power}

Table 4: A summary of the average total energy of the error in each acceleration/deceleration profile, measured using step and ramp deltas for both the GPS and OBDII reference voltages.

\begin{tabular}{|c|c|c|c|c|c|}
\hline & & \multicolumn{2}{|c|}{ GPS } & \multicolumn{2}{|c|}{ OBDII } \\
\hline & & Step & Ramp & Step & Ramp \\
\hline \multirow{4}{*}{ Acc A } & Min & 0.460 & 0.593 & 0.792 & 3.002 \\
\hline & Max & 0.751 & 1.518 & 1.303 & 9.639 \\
\hline & Mean & 0.574 & 1.051 & 1.090 & 5.404 \\
\hline & Stdev & 0.087 & 0.297 & 0.151 & 1.852 \\
\hline \multirow{4}{*}{ Acc B } & Min & 0.448 & 0.687 & 0.810 & 3.238 \\
\hline & Max & 0.748 & 1.974 & 1.366 & 10.338 \\
\hline & Mean & 0.589 & 1.132 & 1.106 & 5.546 \\
\hline & Stdev & 0.086 & 0.366 & 0.168 & 1.764 \\
\hline \multirow{4}{*}{ Acc C } & Min & 0.399 & 0.411 & 0.747 & 3.472 \\
\hline & Max & 0.756 & 1.832 & 1.428 & 8.650 \\
\hline & Mean & 0.532 & 0.979 & 1.064 & 5.585 \\
\hline & Stdev & 0.087 & 0.374 & 0.153 & 1.456 \\
\hline
\end{tabular}

Table 4 shows the minimum, maximum, mean, and standard deviation of the average total energy of the error for each acceleration/deceleration profile. The mean error energy using the GPS velocity reference is mostly lower than for the OBDII velocity reference, and the step delta error energy is always lower than the ramp delta. 


\subsubsection{Corrected Accelerometer Correlation}

Table 5: Summary of the uncorrected acceleration sensor correlation data for each of the smart phone acceleration profiles.

\begin{tabular}{|c|c|c|c|c|}
\hline & & $\operatorname{Acc} \mathbf{A}$ & Acc B & Acc C \\
\hline \multirow{4}{*}{$\operatorname{Acc} \mathbf{A}$} & Max & & 0.884 & 0.896 \\
\hline & Mean & & 0.833 & 0.850 \\
\hline & Min & & 0.756 & 0.795 \\
\hline & StDev & & 0.037 & 0.034 \\
\hline \multirow{4}{*}{ Acc B } & Max & 0.884 & & 0.908 \\
\hline & Mean & 0.833 & & 0.852 \\
\hline & Min & 0.756 & & 0.722 \\
\hline & StDev & 0.037 & & 0.045 \\
\hline \multirow{4}{*}{ Acc C } & Max & 0.896 & 0.908 & \\
\hline & Mean & 0.850 & 0.852 & \\
\hline & Min & 0.795 & 0.722 & \\
\hline & StDev & 0.034 & 0.045 & \\
\hline
\end{tabular}


Table 6: Summary of the corrected acceleration sensor correlation data for each of the smart phone acceleration profiles using the GPS velocity reference and step delta function.

\begin{tabular}{|c|c|c|c|c|}
\hline \multicolumn{1}{|c|}{} & Max & Acc A & Acc B & Acc C \\
\hline \multirow{4}{*}{ Acc A } & Mean & & 0.978 & 0.966 \\
\cline { 2 - 5 } & Min & & 0.967 & 0.954 \\
\cline { 2 - 5 } & StDev & & 0.957 & 0.935 \\
\hline \multirow{4}{*}{ Acc B } & Max & 0.978 & 0.007 & 0.009 \\
\cline { 2 - 5 } & Mean & 0.967 & & 0.967 \\
\cline { 2 - 5 } & Min & 0.957 & & 0.951 \\
\cline { 2 - 5 } & StDev & 0.007 & & 0.934 \\
\hline \multirow{3}{*}{ Acc C } & Max & 0.966 & 0.967 & \\
\cline { 2 - 6 } & Mean & 0.954 & 0.951 & \\
\cline { 2 - 6 } & Min & 0.935 & 0.934 & \\
\cline { 2 - 6 } & StDev & 0.009 & 0.009 & \\
\hline
\end{tabular}

Table 5 and Table 6 contain the minimum, maximum, mean, and standard deviation of the correlation between the corrected and uncorrected measures of acceleration. The corrected accelerations have a significantly higher mean and much narrower standard deviation showing the improvements that have been achieved when the accelerations are recalibrated using the GPS reference and the step delta function. 


\subsection{Implications of the Adaptive Filtering Technique}

Accelerometer sensors that require very low power are now available. Through the use of calibration techniques, they can be deployed in sensor solutions where energy use is a consideration (such as monitoring an individual moving around on foot, rather than in a vehicle) and directly measuring position with a GPS would require high power consumption. This would achieve a higher sampling rate while still optimizing battery use. The use of dynamic calibration would also allow both for longer term deployment
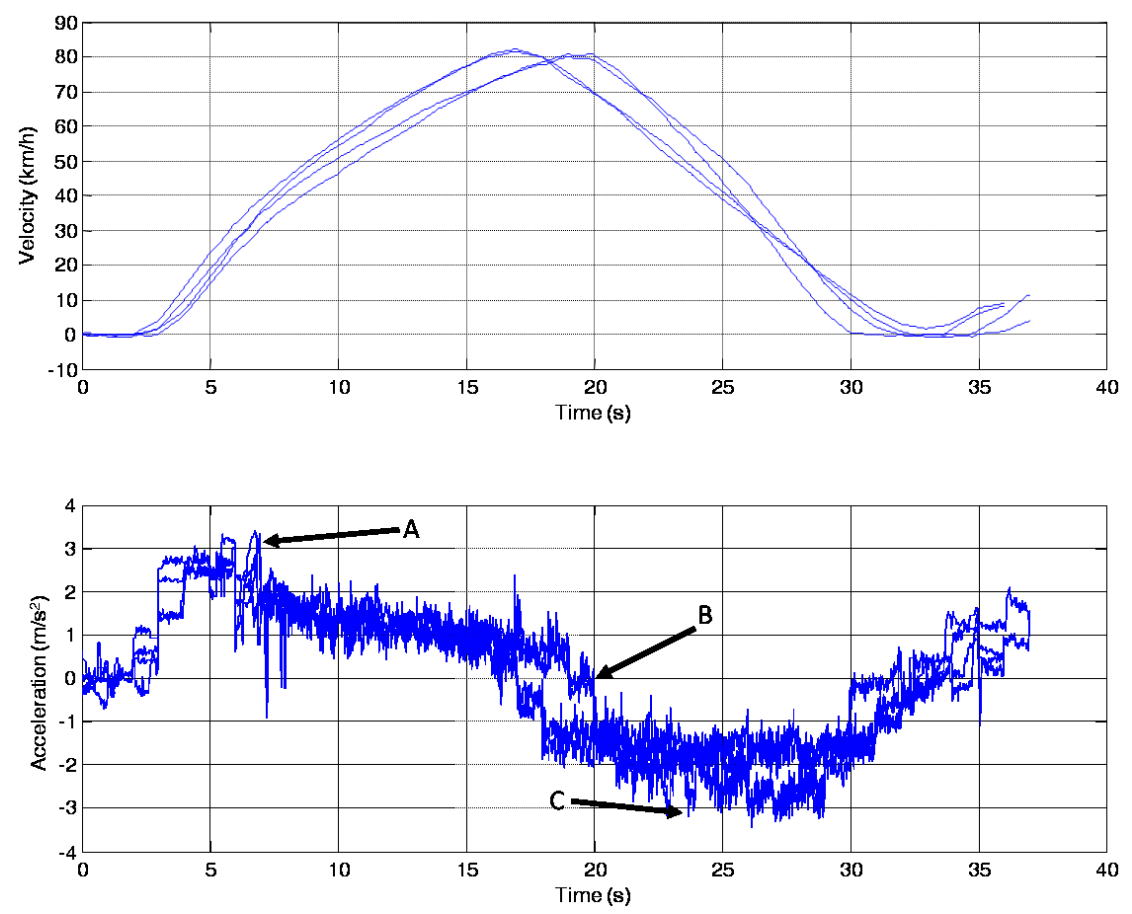

Figure 34: Hard acceleration profile

This figure shows four acceleration profiles in which the vehicle was accelerated to a velocity of $80 \mathrm{~km} / \mathrm{h}$ and stopped. The upper plot shows velocity calculated from corrected acceleration, while the lower plot shows corrected acceleration. A number

of features of interest become clear immediately: point $\mathrm{A}$ indicates the point of maximum acceleration, point $\mathrm{B}$ indicates the point at which the brakes are applied, and point $\mathrm{C}$ indicates the point of maximum deceleration. 
and for measurements performed over longer periods of time. Further, accelerometers are capable of much higher sampling rate, and an accurate measure of acceleration would provide a clearer look at some characteristics of driving behavior. Included in these that are potentially important for establishing a driver signature based on "driver semantics" such as patterns of acceleration/deceleration in different driving scenarios [99]. This will provide a more precise method for tracking changes in behavior that could be correlated with negative health outcomes or increased crash risk. Figure 34 shows a number of acceleration profiles in which the vehicle was accelerated to a velocity of approximately $80 \mathrm{~km} / \mathrm{h}$ along a straight road, and then returned to a stop. The upper plot shows velocity calculated from corrected acceleration, while the lower plot shows corrected acceleration. A number of potentially interesting features in the lower plot are immediately evident, and have been pointed out in the figure. Point A shows the maximum acceleration achieved, point $\mathrm{B}$ indicates the time at which the brakes are applied, and point $\mathrm{C}$ indicates the maximum deceleration achieved. The point of maximum acceleration occurs shortly after setting off, then tapers to the maximum velocity, at which point the brakes are applied. The deceleration curve is roughly a mirror of the acceleration curve, with the point of maximum deceleration occurring just before the vehicle returns to a stop. Figure 35 shows a number of acceleration profiles in which the vehicle was accelerated to a maximum velocity of less than $70 \mathrm{~km} / \mathrm{h}$, and then returned to a stop. In these softer accelerations, the points of interest identified in the previous figure are less clearly visible, but still present. Figure 36 is a direct comparison of the mean values of the acceleration profiles shown in the preceding two figures. Here, the differences between the two sets of profiles become clearer. The point of maximum acceleration (point A) 
appears to occur at the same time for both hard and soft accelerations, though the maximum hard acceleration is clearly higher than the soft. Interestingly, there is a noticeable dip in both waveforms immediately after the maximum acceleration is achieved. This may suggest a gear change during acceleration. The time at which the gas pedal is released (point B) appears to be the same for each set, but the brakes are depressed almost immediately in the hard acceleration, while the soft acceleration waveform remains at roughly 0 for two to three seconds before beginning to decelerate. This is interesting because it demonstrates that there is a clear difference in the shape of the waveform between more leisurely braking (as in the softer acceleration profile) and
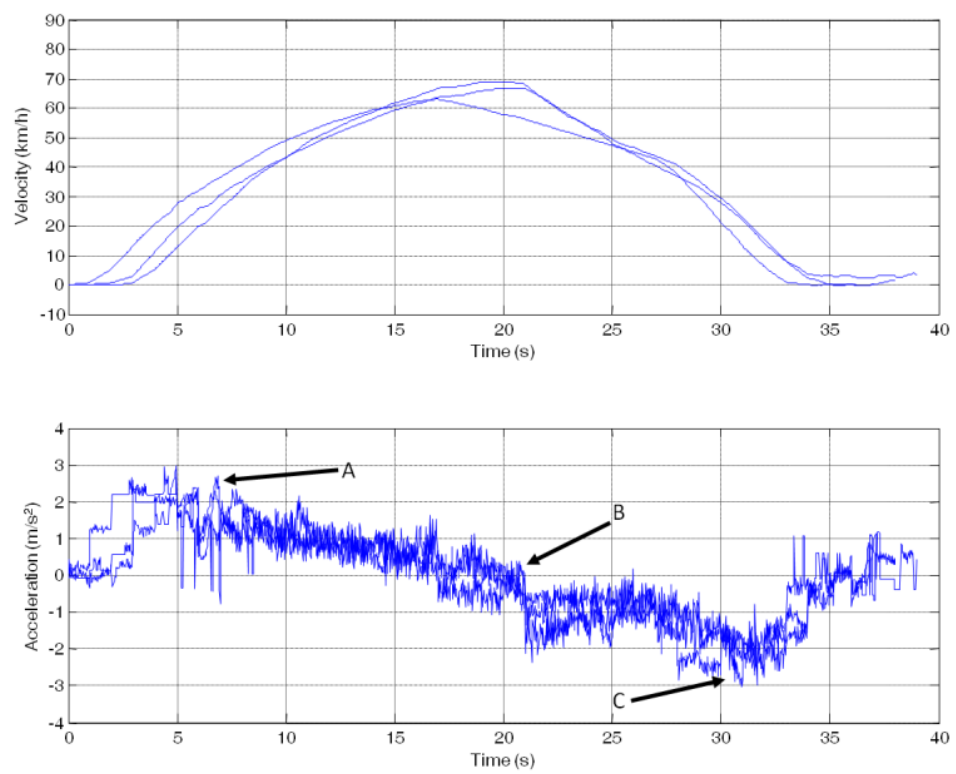

Figure 35: Soft acceleration profile

This figure shows four acceleration profiles in which the vehicle was accelerated to a velocity of less than $70 \mathrm{~km} / \mathrm{h}$ and returned to a stop. The upper plot shows velocity calculated from corrected acceleration, while the lower plot shows corrected acceleration. As in Figure 34, an initial maximum acceleration (point A) tapers over time until the brakes are applied (point B), followed by steadily increasing deceleration to a minimum (point $\mathrm{C}$ ) before the vehicle comes to rest. 
braking under pressure (as in the harder acceleration profile). Since this experiment was performed on a single, short stretch of rural road, there was less stopping space available after achieving the higher speed. This was closer to emergency braking than in the softer acceleration profiles, where we can see two to three seconds of coasting time. Finally, the value of maximum deceleration (point C) seems to actually be similar for both the hard and soft acceleration profiles (but, as mentioned previously, the brakes are clearly more forcefully applied for the hard acceleration profile). Each of these features could be used in the development of the driver signature and could play a role in generating useful information about the overall driving capability of an individual (for instance slowing of reaction times could be measured through investigation of the shape of the deceleration
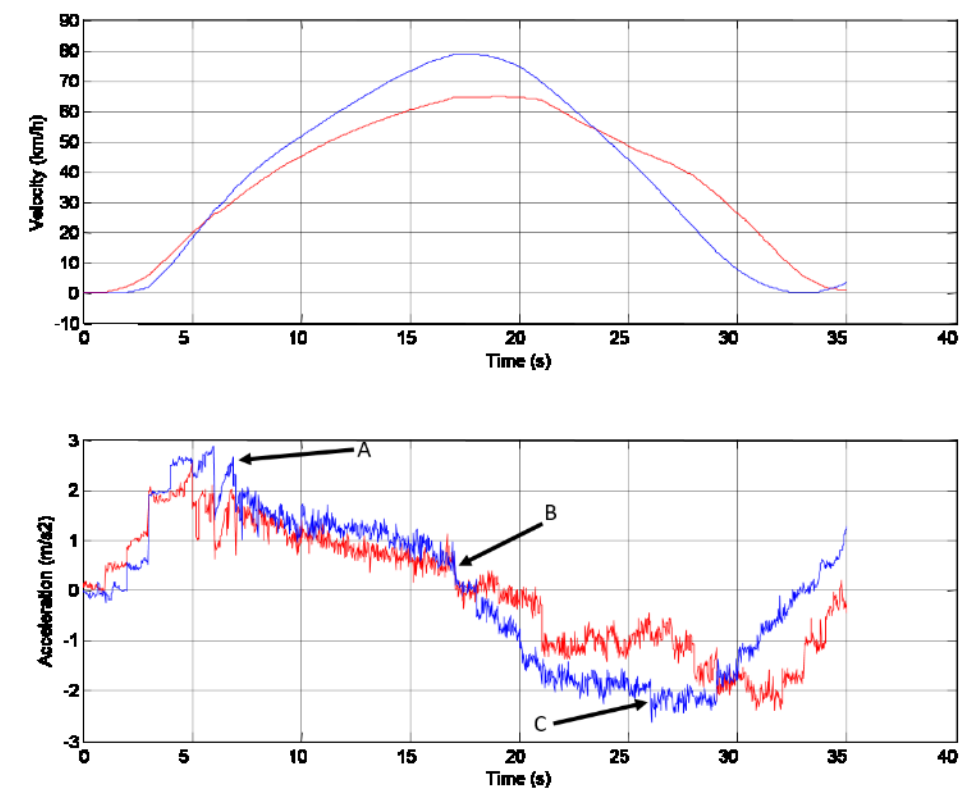

Figure 36: comparison of mean hard acceleration vs soft acceleration

This figure shows a comparison of the mean hard acceleration (blue) vs the mean soft acceleration (red). Differences between the three points of interest become immediately clear. The point of maximum acceleration is clearly higher in the hard acceleration than the soft. The maximum deceleration values are similar, but the average deceleration is stronger in the hard line. 
curve, with later, more severe braking expected as reaction times decrease). Further study could identify patterns of driving behavior that are associated with increased collision risk, and used by physicians or policymakers in performing driving risk assessment to determine a person's ability to drive.

GPS sensors typically have a fairly low accuracy, and can often only determine the location of the receiver to within a few meters. This inaccuracy manifests as a lognormal distribution. As demonstrated in Figure 37, when the GPS receiver is held still and measurements are taken repeatedly, the value will fluctuate, often by a number of meters, around the actual location of the device. This means that higher sampling rates will actually effectively have higher error, especially when the device is being used to track movement, as shown in Figure 38. For example, if the device is moving at 1 meter

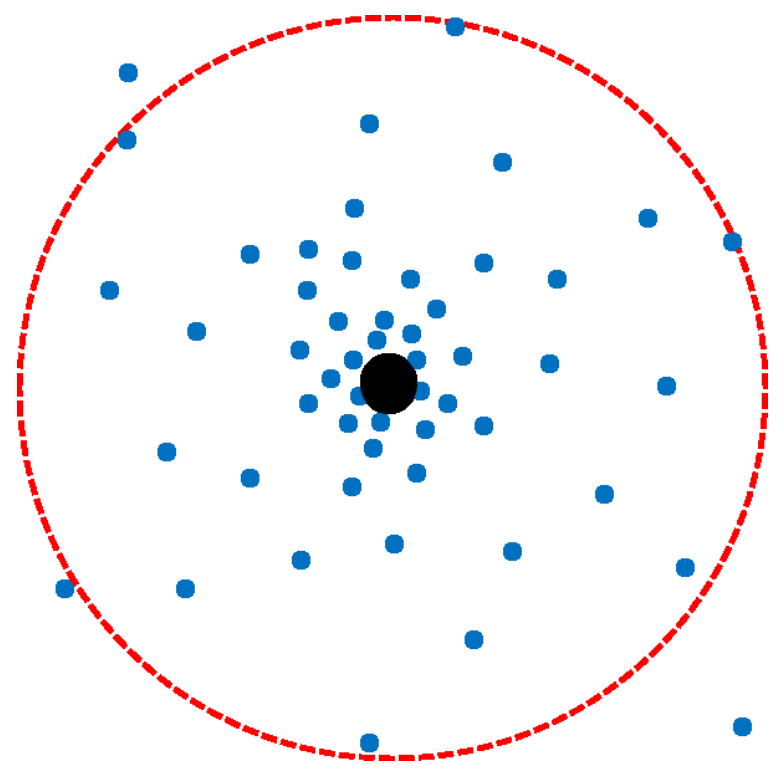

Figure 37: GPS inaccuracy over repeated measures

Repeated GPS measures relative to an absolute position. The black spot represents the actual location of the device, while the blue dots show the result of repeated measurements. The red dashed circle represents the $95 \%$ confidence radius. The distribution of points is lognormal in nature 
per second, and sampling at $1 \mathrm{~Hz}$ with a 95\% accuracy of 3 meters, the distance covered each measurement could very easily be lost in the noise, producing an inaccurate measure of the movement (Figure 39). If the parameters of the measurement remained the same and the sampling rate were reduced to $0.1 \mathrm{~Hz}$, the measurement accuracy would be improved significantly (Figure 40). The adaptive filtering technique presented here allows the advantages offered by low GPS sampling rates to be exploited and combined with the high sensitivity and sampling rate of the accelerometer to improve the measurement of the position, velocity, and acceleration. 


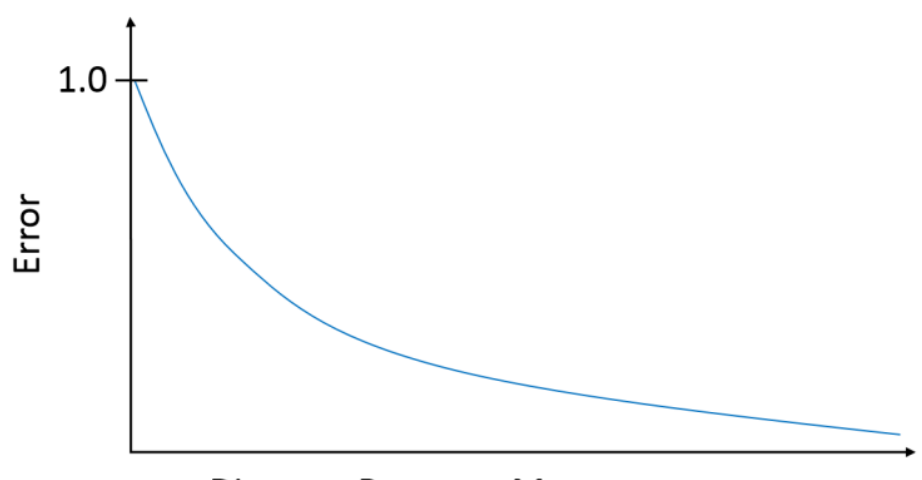

Distance Between Measurements

Figure 38: Illustration showing relative GPS error vs distance between measurements

As the distance between measurements increases, the effective error decreases.

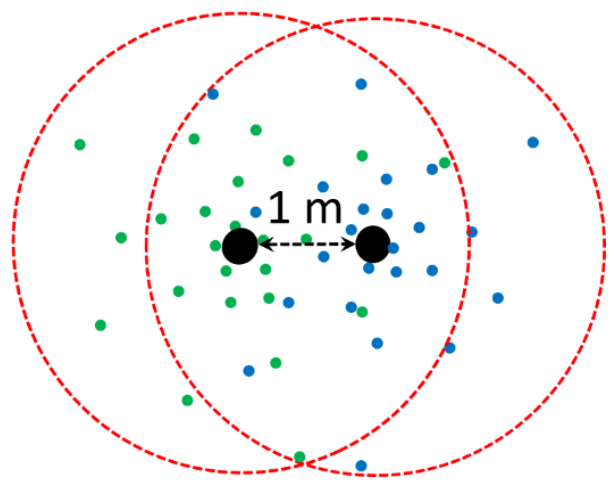

\section{Figure 39: GPS measurement over a short distance}

When the distance traversed between measurements is short, each new point can be entirely indistinguishable from noise around the previous point.

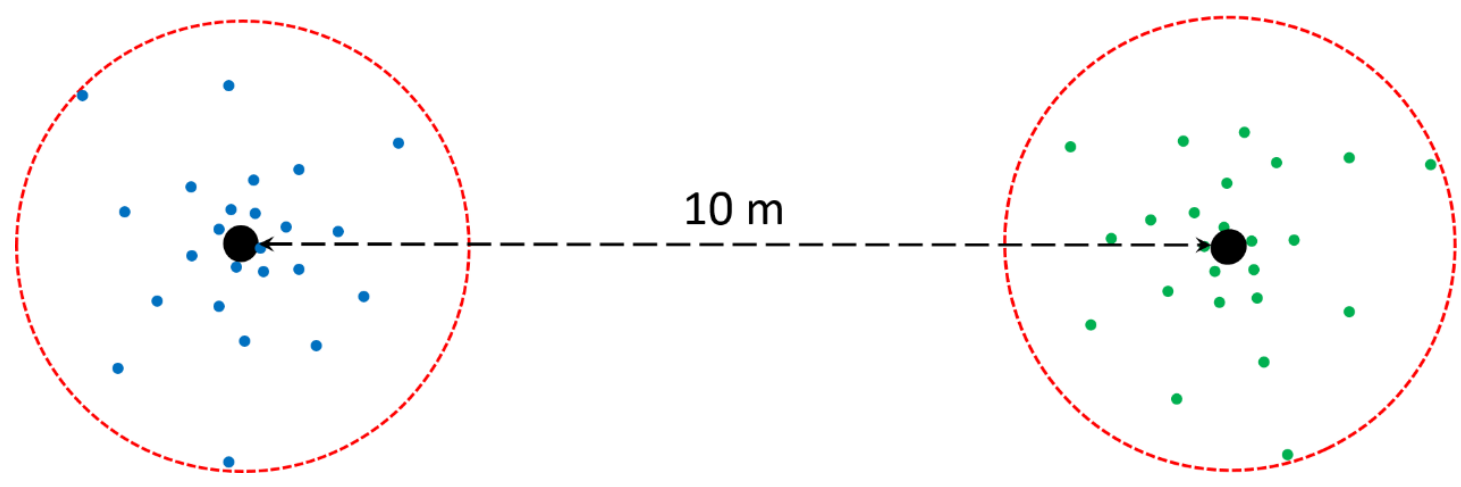

Figure 40: GPS measurement over a longer distance

When measuring longer distances between points, noise contributes much less than the signal to the measurement. 


\subsection{Conclusion}

The RPM system implemented in the preceding chapters was employed to collect acceleration data from a test vehicle so that its usefulness in developing a driver signautre could be investigated. This chapter has described the methods used for data collection and has detailed the adaptive calibration algorithm that has been developed. It has also shown that using a reference velocity signal, such as a direct velocity measurement through an OBDII interface or a velocity derived from a GPS sensor, allows dynamic drift correction of accelerometer data, even over long time periods. This correction allows the use of accelerometers in continuous monitoring of driving behavior. The chapter has also shown that a ramp based delta function causes unacceptable oscillations in the calibrated signal while the step function provides improved performance. Further, it has identified several advantages offered by this method of adaptive filtering over methods already employed, and describes how it could be utilized in combination with a GPS receiver to improve measurements of all three of the basic measures of motion: acceleration, velocity, and position. It has also identified a number of features of driving behavior and has discussed how the corrected acceleration data could be used to assess driver risk. 


\section{Chapter 7:}

\section{Conclusions}

\subsection{Thesis Conclusions}

The primary objectives of this thesis were: first, to design an RPM system that could be installed either in a car or in the home, and second, to make use of the RPM system to begin the process of developing a tool to more fairly assess the competence of elderly drivers. An RPM system was implemented that consisted of a dedicated local node (with software built on top of the QNX Neutrino RTOS for compatibility with vehicle installation) used for data acquisition and high-priority signal processing, and a central node that is fed by a number of local nodes and is used as a central hub for longterm data storage and trend analysis. This RPM system was then employed to collect data from three accelerometers, which employed an adaptive calibration algorithm so they could be used to assist in uniquely identifying drivers' "driving signatures". Driver identification is an important first step in developing the tools to effectively assess driver competence, as, especially with shared vehicles, it is important to correctly discriminate between drivers. Additionally, the same features that make up a driver's signature could be important in that they may be key to determining a driver's performance, or in that they may change in predictable ways with worsening cognitive or physical impairment. 
Finally, driving ability is a very high order function, and is correlated with other highorder functions such as mobility, attention, planning, and physical function. The RPM system that was developed was not simply designed for use in the car to monitor driving ability, however. It was also developed to be used in the home to monitor all aspects of overall health, since function in the home can also provide important details for making decisions regarding the independence of older adults. Finally, the creation of such a highly generalizable RPM system will allow truly continuous monitoring by a single, coherent system in the future.

\subsection{Thesis Contributions}

The following is a list of research contributions that were made during the development of this thesis; each contribution will be explained in more detail in the following chapters:

1. The first contribution was the design and development of a full framework for a remote monitoring system. This system is compatible with the on-board operating system of many types of vehicles, with several types of powerful microcontrollers, and with Blackberry smartphones, making it an effective system for 24-hour automatic monitoring in any environment.

a. M. Rockwood, K. Sullivan, R. Goubran, "Remote Patient Monitoring Using the QNX Real Time Operating System," presented at The $3^{\text {rd }}$ annual conference of Healthcare Support through Information Technology Enhancements (hSITE), Montreal, QC, 2013. 
b. M. Rockwood, V. Joshi, K. Sullivan, R. Goubran, "Using a Real-Time Operating System for Multitasking in Remote Patient Monitoring," in proc. 2014 IEEE International Symposium on Medical Measurements and Applications (MeMeA), pp. 475-479, 2014.

2. The second contribution was the development of an experiment and data acquisition application to collect acceleration data from a vehicle outfitted with Candrive sensors. The goal was to use the data to assist in the development of a 'driver signature' that could be used to track driver performance and ultimately help inform decisions about driving cessation among elderly people.

a. B. Wallace, M. Rockwood, R. Goubran, F. Knoefel, S. Marshall, M. Porter. "Measurement of Vehicle Acceleration in Studies of Older Drivers from GPS Position and OBDII Velocity Sensors," submitted to 2015 IEEE International Symposium on Medical Measurements and Applications (MeMeA).

3. The third contribution was to design and implement an adaptive filtering algorithm to calibrate the accelerometer data using two measures of the car's velocity as reference values. This allows the full use of the higher sampling rate and lower power accelerometer without having to manage the complex drift and integration error issues normally associated with accelerometer measurements.

a. M. Rockwood, B. Wallace, R. Goubran, F. Knoefel, S. Marshall. "Adaptive Drift Calibration of Accelerometers with Direct Velocity Measurements," accepted at 2015 IEEE International Instrumentation and Measurement Technology Conference (I2MTC). 


\subsection{Future Research}

The RPM system was designed with extensibility as one of its top priorities. The current system only makes use of simulated sensors and the sensor array built into a smart phone, but the local node in fact accepts data in any format, and can be easily extended to handle an arbitrary number and type of sensors. An immediate goal of future work would be to accept and parse data from the pressure mats used by many other researchers in our group. This would then immediately allow the implementation of all existing pressure mat algorithms in real time, enabling research into the clinical usefulness of each. Further, integration with all of the research group's sensor equipment could improve data collection efficiency in a number of ways. Purchasing server space for a central node and installing a number of local nodes in locations where our group is conducting on-site research would eliminate the need for periodic site visits for collection and replacement of memory cards, and would allow immediate notification of the failure of any sensor, thereby preventing issues with data loss should there be a hardware problem between site visits.

With regard to accelerometer calibration, there are a number of immediately available avenues for further exploration. Firstly, the existing adaptive calibration algorithm could be expanded to specifically include adaptive scale calibration to fully account for variable scaling issues with the accelerometer signals over long duration recording. Secondly, the velocity signal used for reference was sampled at $1 \mathrm{~Hz}$, but there is no reason to believe this is the minimum achievable reference sampling rate. Decreasing the reference sampling rate is desirable because many other methods of 
determining velocity (such as by calculation from GPS position) are battery-intensive operations, and reducing their time active will improve battery life in mobile applications. Finally, this method of correcting acceleration could be explored in combination with GPS calculated velocity for characterizing activity at lower velocities, such as walking or running.

Importantly, the work completed here demonstrates that this approach allows for a number of higher order functions to be tracked simultaneously, with priority given to monitoring those most closely associated with loss of independence or important negative health trends. Longer term development could see the RPM system, and the sensor equipment connected to it, used collectively to monitor all high order functions in real time. This would make a complete picture of the overall health of a patient available without the need to completely customize the system for each patient's specific set of health problems. In fact, other members of this research group have already begun the process of investigating the link between mobility (another high order function) and overall health, so avenues for collaboration already exist. 


\section{References}

[1] The Special Senate Committee on Aging. (2009). Canada's Aging Population Seizing the Opportunity. [Online] Available: http://www.parl.gc.ca/content/sen/committee/402/agei/rep/agingfinalreporte.pdf

[2] M. Hoover, M. Rotermann, C. Sanmartin, J. Bernier, "Validation of an index to estimate the prevalence of frailty among community-dwelling seniors," Health Rep, vol. 24, no. 9, pp. 10-17, Sep. 2013.

[3] C. H. Snyder, "Dementia and driving: autonomy versus safety," J. Am. Acad. Nurse Pract., vol. 17, no. 10, pp. 393-402, Oct. 2005.

[4] D. W. Molloy, R. M. Clarnette, E. A. Braun, M. R. Eisemann, B. Sneiderman, "Decision making in the incompetent elderly: 'The Daughter from California syndrome'," J Am Geriatr Soc, vol. 39, no. 4, pp. 396-399, Apr. 1991.

[5] B. G. Celler, E. D. Ilsar, W. Earnshaw, "Preliminary results of a pilot project on remote monitoring of functional health status in the home," in Proc. 18th Annual International Conference of the IEEE Engineering in Medicine and Biology Society, 1996, pp. 63-64.

[6] CBC News. (2002). Inquest told elderly driver had just passed test [Online]. Available: http://www.cbc.ca/news/canada/inquest-told-elderly-driver-hadjust-passed-test-1.320636

[7] M. K. Andrew, J. M. Keefe, "Social vulnerability from a social ecology perspective: a cohort study of older adults from the National Population Health Survey of Canada," BMC Geriatr., pp. 14:90, Aug. 2014.

[8] B. R. Ott, J. D. Davis, G. D. Papandonatos, S. Hewitt, E. K. Festa, W. C. Heindel, C. A. Snellgrove, D. B. Carr, "Assessment of driving-related skills prediction of unsafe driving in older adults in the office setting". $J \mathrm{Am}$ Geriatr Soc.,vol. 61, no. 7, pp. 1164-1169, Jul. 2013. 
[9] D. B. Carr, B. R. Ott, "The older adults driver with cognitive impairment: 'it's a very frustrating life'," JAMA, vol. 303, no. 16, pp. 1632-1641, Apr. 2010.

[10] J. N. Morris, E. P. Howard, B. E. Fries, R. Berkowitz, B. Goldman, D. David. "Using the community health assessment to screen for continued driving," Accid. Anal. Prev., vol. 63, pp. 104-110, Feb. 2014.

[11] E. L. Aufman, M. D. Bland, P. P. Barco, D. B. Carr, C. E. Lang, "Predictors of return to driving after stroke," Am. J. Phys. Med. Rehabil., vol. 92, no. 7, pp. 627-634, Jul. 2013.

[12] S. Seiler, H. Schmidt, A. Lechner, T. Benke, G. Sanin, G. Ransmayr, R. Lehner, P. Dal-Bianco, P. Santer, P. Linortner, C. Eggers, B. Haider, M. Uranues, J. Marksteiner, F. Leblhuber, P. Kapeller, C. Bancher, R. Schmidt, PRODEM Study Group, "Driving cessation and dementia: results of the prospective registry on dementia in Austria (PRODEM)," PLoS One, vol. 7, no. 12, pp. 52710, Dec. 2012.

[13] J. Marmeleira, I. Ferreira, F. Melo, M. Godinho, "Associations of physical activity with driving-related cognitive abilities in older drivers: an exploratory study," Percept Mot Skills, vol. 115, no. 2, pp. 521-533, Oct. 2012.

[14] K. Motta, H. Lee, T. Falkmer, "Post-stroke driving: examining the effect of executive dysfunction," J Safety Res, vol. 49, pp. 33-38, Jun. 2014.

[15] P. A. Hoggarth, C. R. Innes, J. C. Dalrymple-Alford, R. D. Jones, "Predicting on-road assessment pass and fail outcomes in older drivers with cognitive impairment using a battery of computerized sensory-motor and cognitive tests," J. Am. Geriatr. Soc., vol. 61, no. 12, pp. 2192-2198, Dec. 2013.

[16] P. Lacherez, J. M. Wood, K. J. Anstey, S. R. Lord, "Sensorimotor and postural control factors associated with driving safety in a communitydwelling older driver population," J. Gerontol. A. Biol. Sci. Med. Sci., vol. 69, no. 2, pp. 240-244, Feb. 2014.

[17] B. R. Ott, J. D. Davis, G. D. Papandonatos, S. Hewitt, E. K. Festa, W. C. Heindel, C. A. Snellgrove, D. B. Carr, "Assessment of driving-related skills prediction of unsafe driving in older adults in the office setting," $\mathrm{J}$. Am.

Geriatr. Soc., vol. 61, no. 7, pp. 1164-1169, Jul. 2013. 
[18] C. R. Innes, D. Lee, C. Chen, A. M. Ponder-Sutton, R. D. Jones, "Different models for predicting driving performance in people with brain disorders," in Proc. IEEE Eng. Med. Biol. Soc. (EMBS), 2010, pp. 5226-5229.

[19] D. H. Davis, M. R. Rockwood, A. B. Mitnitski, K. Rockwood, "Impairments in mobility and balance in relation to frailty," Arch. Gerontol. Geriatr., vol. 53, no. 1, pp. 79-83, Jul. 2011.

[20] O. Theou, M. R. Rockwood, A. Mitnitski, K. Rockwood, "Disability and comorbidity in relation to frailty: how much do they overlap?," Arch. Gerontol. Geriatr., vol. 55, no. 2, pp. 1-8, Sep. 2012.

[21] M. R. Rockwood, S. E. Howlett, K. Rockwood, "Orthostatic hypotension $(\mathrm{OH})$ and mortality in relation to age, blood pressure and frailty," Arch. Gerontol. Geriatr., vol. 54, no. 3, pp. 255-260, May 2012.

[22] J. Gaudet, M. F. Bélanger, H. Corriveau, S. Mekary, D. Hay, M. J. Johnson, "Investigating the autonomic nervous system and cognitive functions as potential mediators of an association between cardiovascular disease and driving performance," Can. J. Physiol. Pharmacol., vol. 91, no. 5, pp. 346352, May 2013.

[23] P. Pawar, V. Jones, B. F. van Beijnum, "A framework for the comparison of mobile patient monitoring systems," Journal of biomedical informatics, vol. 45, no. 3, pp. 544-556, Jun. 2012.

[24] B. J. Wakefield, R. J. Koopman, L. E. Keplinger, M. Bomar, B. Bernt, J. L. Johanning, R. L. Kruse, J. W. Davis, D. S. Wakefield, D. R. Mehr, "Effect of Home Telemonitoring on Glycemic and Blood Pressure Control in Primary Care Clinic Patients with Diabetes," Telemed. J. E. Health., vol. 20, no. 3, pp. 199-205, Jan. 2014.

[25] P. B. Angood, R. Satava, C. Doarn, R. Merrell, "Telemedicine at the top of the world: the 1998 and 1999 Everest extreme expeditions," Telemed. J. E. Health, vol. 6, no. 3, pp. 315-325, Oct. 2000.

[26] S. McLean, A. Susannah, K. Cresswell, U. Nurmatov, "The impact of telehealthcare on the quality and safety of care: a systematic overview," PloS one, vol. 8, no. 8, pp. 71238, Aug. 2013.

[27] C. E. Chronaki, P. Vardas, "Remote monitoring costs, benefits, and reimbursement: a European perspective," Europace, vol. 15, no. 1, pp. 59- 
64, Jun. 2013.

[28] J. M. Pericás, J. Aibar, N. Soler, A. López-Soto, C. Sanclemente-Ansó, X. Bosch, "Should alternatives to conventional hospitalisation be promoted in an era of financial constraint?," Eur. J. Clin. Invest., vol. 43, no. 6, pp. 602615, Jun. 2013.

[29] F. Sposaro, G. Tyson, "Geriatric medical application suite on a sweet phone," in Proc. First AMA IEEE Med. Technology Conf. on Individualized Healthcare, 2010, pp. 1.

[30] M. C. Barroso, G. P. Esteves, T. P. Nunes, L. M. Silva, A. C. Faria, P. L. Melo, "A telemedicine instrument for remote evaluation of tremor: design and initial applications in fatigue and patients with Parkinson's

Disease," BioMedical Engineering OnLine, vol. 10, no. 1, pp. 14, Feb. 2011.

[31] S. I. Chaudhry, J. A. Mattera, J. P. Curtis, J. A. Spertus, J. Herrin, Z. Lin, C. O. Phillips, B. V. Hodshon, L. S. Cooper, H. M. Krumhotz, "Telemonitoring in patients with heart failure," New England Journal of Medicine, vol. 363, no. 24, pp. 2301-2309, Dec. 2010.

[32] F. Fontana, B. Kennedy, V. Longo, D. Seals, S. Melov, "Medical research: Treat ageing," Nature, vol. 511, pp. 405-407, Jul. 2014.

[33] P. G. Jarrett, K. Rockwood, D. Carver, P. Stolee, S. Cosway, "Illness presentation in elderly patients," Arch. Intern. Med., vol. 155, no. 10, pp. 1060-1064, May 1995.

[34] A. Mihailidis, A. Cockburn, C. Longley, J. Boger, "The acceptability of home monitoring technology among community-dwelling older adults and baby boomers," Assist. Technol., vol. 20, no. 1, pp. 1-12, Apr. 2008.

[35] S. Czaja, S. Beach, N. Charness, R. Schulz, "Older Adults and the Adoption of Healthcare Technology: Opportunities and Challenges," Technologies for Active Aging, vol. 9, A. Sixsmith and G. Gutman, Eds. Springer US, 2013, pp. 27-46.

[36] K. L. Courtney, G. Demiris, M. Rantz, M. Skubic, "Needing smart home technologies: the perspectives of older adults in continuing care retirement communities," Inform. Prim. Care, vol. 16, no. 3 pp. 195-201, Feb. 2008.

[37] D. W. Eby, N. M. Silverstein, L. J. Molnar, D. LeBlanc, G. Adler, "Driving behaviours in early stage dementia: a study using in-vehicle technology," 
Accident Anal. Prev., vol. 49, pp. 330-337, Nov. 2012.

[38] D. A. Breen, D. P. Breen, J. W. Moore, P. A. Breen, D. O’Neill, "Driving and dementia," Br. Med. J., vol. 334 pp. 1365-1369, Apr. 2007.

[39] D. J. Iverson, G. S. Gronseth, M. A. Reger, S. Classen, R. M. Dubinsky, M. Rizzo, "Practice parameter update: evaluation and management of driving risk in dementia," Neurology, vol. 74, pp. 1316-1324, Apr. 2010.

[40] A. E. Dickerson, D. B. Meuel, C. D. Ridenour, K. Cooper, "Assessment tools predicting fitness to drive in older adults: a systematic review," Am. J. Occup. Ther., vol. 68, no. 6, pp. 670-680, Nov. 2014.

[41] M. T. Silva, J. Laks, E. Engelhardt, "Neuropsychological tests and driving in dementia: a review of the recent literature," Rev. Assoc. Med. Bras., vol. 55, pp. 484-488, Jul. 2009.

[42] J. M. Ducheck, L. Hunt, K. Ball, V. Buckles, J. C. Morris, "The role of selective attention in driving and dementia of the Alzheimer type," Alzheimer Dis Assoc Disord, vol. 11, pp. 48-56, Jun. 1997.

[43] B. M. Dobbs, S. S. Shergill, "How effective is the Trail Making Test (Parts A and B) in identifying cognitively impaired drivers?," Age Ageing, vol. 42, no. 5, pp. 577-81, Sep. 2013.

[44] L. B. Brown, B. R. Ott, "Driving and dementia: a review of the literature," $J$ Geriatr Psychiatry Neurol, vol. 17, pp. 232-240, Dec. 2004.

[45] F. J. Molnar, A. Patel, S. C. Marshall, M. Man-Son-Hing, K. G. Wilson, "Clinical utility of office-based cognitive predictors of fitness to drive in persons with dementia: a systematic review," J. Am. Geriatr. Soc., vol. 54 pp. 1809-1824, Dec. 2006.

[46] P. M. Butala, Y. Zhang, T. D. C. Little, "Wireless system for monitoring and real-time classification of functional activity," in Proc. International Conference on Communications Systems and Networks (COMSNETS), 2012, pp. 1-5.

[47] P. Gupta, T. Dallas, "Feature Selection and Activity Recognition System Using a Single Triaxial Accelerometer," IEEE Transactions on Biomedical Engineering, vol. 61, no. 6, pp. 1780-86, Jun. 2014. 
[48] Harding P, Holland AE, Delany C, Hinman RS. "Do activity levels increase after total hip and knee arthroplasty?," Clin. Orthop. Relat. Res., vol. 472, no. 5, pp. 1502-1511, May 2014.

[49] M. Yoneyama, Y. Kurihara, K. Watanabe, H. Mitoma, "Accelerometrybased gait analysis and its application to Parkinson's disease assessmentpart 2: a new measure for quantifying walking behavior," IEEE Trans. Neural Syst. Rehabil. Eng., vol. 21, no. 6, pp. 999-1005, Nov. 2013.

[50] E, Calore, F. Pedersini, I. Frosio, "Accelerometer based horizon and keystone perspective correction," in Proc. Instrumentation and Measurement Technology Conference (I2MTC), 2012 IEEE International, 2012, pp. 205-209.

[51] I. Frosio, F. Pedersini, N. Alberto Borghese, "Autocalibration of MEMS accelerometers," IEEE Transactions on Instrumentation and Measurement, vol. 58, no. 6, pp. 2034-2041, May 2009.

[52] E. Timpson, T. Engel, "Calibrating accelerometers using an Electromagnetic Launcher," in Proc. Instrumentation and Measurement Technology Conference (I2MTC), 2012 IEEE International, 2012, pp. 1027-1032.

[53] W. Guan, X. Meng, X. Dong, "Calibration of accelerometer with multicomponent inputs," in Proc. Instrumentation and Measurement Technology Conference (I2MTC), 2014 IEEE International, 2014, pp. 1619.

[54] T. Beravs, J. Podobnik, M. Munih, "Three-Axial Accelerometer Calibration Using Kalman Filter Covariance Matrix for Online Estimation of Optimal Sensor Orientation," IEEE Transactions on Instrumentation and Measurement, vol. 61, no. 9, pp. 2501-2511, Aug. 2012.

[55] S. Won, F. Golnaraghi, "A Triaxial Accelerometer Calibration Method Using a Mathematical Model," IEEE Transactions on Instrumentation and Measurement, vol. 59, no. 8, pp. 2144-2153, Jul. 2010.

[56] C. Wan-Young, A. Purwar, A. Sharma, "Frequency domain approach for activity classification using accelerometer," in Proc. Engineering in Medicine and Biology Society (EMBS), 2008. 30th Annual International Conference of the IEEE, 2008, pp. 1120-1123. 
[57] J. L. Carus, V. Pelaez, S. Garcia, M. A. Fernandez, G. Diaz, E. Alvarez, “A Non-invasive and Autonomous Physical Activity Measurement System for the Elderly," in Proc.10th International Conference on Ubiquitous Intelligence and Computing and 10th International Conference on Autonomic and Trusted Computing (UIC/ATC), 2013, pp. 619-624.

[58] I. C. Gyllensten, A. G. Bonomi, "Identifying Types of Physical Activity With a Single Accelerometer: Evaluating Laboratory-trained Algorithms in Daily Life," IEEE Transactions on Biomedical Engineering, vol. 58, no. 9, pp. 2656-2663, Sep. 2011.

[59] S. Marshall, et al. "Protocol for Candrive II/Ozcandrive, a multicentre prospective older driver cohort study," Accident Analysis \& Prevention, vol. 61, pp. 245-252, Dec. 2013.

[60] S. Marshall, et al. "The Canadian Safe Driving Study-phase I pilot: Examining potential logistical barriers to the full cohort study," Accident Analysis \& Prevention, vol. 61, pp. 236-244, Dec. 2013.

[61] D. Eby, et al. "Driving behaviors in early stage dementia: A study using invehicle technology," Accident Analysis \& Prevention, vol. 49, pp. 330-337, Nov. 2012.

[62] R. Wallace, R. Goubran, F Knoefel, "Measurement of Driving Routes and Correlation to Optimal Navigation Paths," in Proc. 2013 IEEE International Instrumentation and Measuremnt Conference (I2MTC), 2013, pp. 14651470.

[63] C. Barrios, Y. Motai, "Improving Estimation of Vehicle's Trajectory Using the Latest Global Positioning System With Kalman Filtering," IEEE Transactions on Instrumentation and Measurement, vol. 60, no. 12, pp. 3747-3755, Dec. 2011.

[64] R. Wallace, R. Goubran, F. Knoefel, "Measurement of Signal Use and Vehicle Turns as Indication of Driver Cognition," in Proc. IEEE Engineering in Medicine and Biology Society (EMBC), 36th Annual International Conference of the, 2014, pp. 3747-3750.

[65] A. S. Hussein, W. M. Omar, L. Xue M. Ati, "Efficient Chronic Disease Diagnosis prediction and recommendation system," in Proc. 2012 IEEE EMBS Conference on Biomedical Engineering and Sciences (IECBES), 2012, pp. 209-214. 
[66] A. Kanakis, B. Malik, M. Benaissa, "Low Cost Universal Remote Patient Monitoring System," in Proc. 2012 IEEE 14th International Conference on High Performance Computing and Communication \& 2012 IEEE 9th International Conference on Embedded Software and Systems (HPCCICESS), 2012, pp. 1587-1591.

[67] T. C. Jannett, S. Prashanth, S. Mishra, V. Ved, A. Mangalvedhekar, J. Deshpande, "An intelligent telemedicine system for remote spirometric monitoring," in Proc. Thirty-Fourth Southeastern Symposium on System Theory, 2002, pp. 53-56.

[68] C. Rotariu, V. Manta, H. Costin, "Wireless remote monitoring system for patients with cardiac pacemakers," 2012 International Conference and Exposition on Electrical and Power Engineering (EPE), 2012, pp. 845-848.

[69] C. Rotariu, D. Arotaritei, V. Manta, "Wireless system for remote monitoring of atrial fibrillation," in Proc. 2012 5th European DSP Education and Research Conference (EDERC), 2012, pp. 129-133.

[70] H. Mansor, M. H. A. Shukor, S. S. Meskam, N. Q. A. M Rusli, N. S. Zamery, "Body temperature measurement for remote health monitoring system," in Proc. 2013 IEEE International Conference on Smart Instrumentation, Measurement and Applications (ICSIMA), 2013, pp. 1-5.

[71] S. Navaneethakrishnan, R. Mahendar, "Remote patient monitoring system for rural population using ultra low power embedded system," in Proc. 2014 International Conference on Computer Communication and Informatics (ICCCI), 2014, pp. 1-5.

[72] D. Goyal, J. Bhaskar, P. Singh, "Designing the low cost patient monitoring device (LCPMD) \& Ubiquitous based Remote Health Monitoring and Health Management System using Tablet PC," in Proc. 2012 2nd IEEE International Conference on Parallel Distributed and Grid Computing (PDGC), 2012, pp. 7-11.

[73] O. Rawashdeh, W. Sa'deh, M. Rawashdeh, Q. Guangzhi, M. Ferrari, B. Harrison, R. Hammond, M. Maddens, "Development of a low-cost fall intervention system for hospitalized dementia patients," in Proc. 2012 IEEE International Conference on Electro/Information Technology (EIT), 2012, pp. 1-7. 
[74] L. Chung-Chih, C. Ming-Jang, H. Chun-Chieh, L. Ren-Guey, T. Yuh-Show, "Wireless Health Care Service System for Elderly With Dementia," IEEE Transactions on Information Technology in Biomedicine, vol. 10, no. 4, pp. 696-704, Oct. 2006.

[75] M. M. Baig, H. Gholamhosseini, "Smart Health Monitoring Systems: An Overview of Design and Modeling," Journal of Medical Systems, vol. 37, no. 2, pp. 1-14, Apr. 2013.

[76] B. L. McLaughlin, L. J. Mariano, S. R. Prakash, A. L. Kindle, A. Czarnecki, "An Electroencephalographic Recording Platform for Real-Time Seizure Detection," in Proc. IEEE Int. Conf. Eng. in Med. and Bio. Soc. (EMBS), San Diego, USA, 2012, pp. 874-878.

[77] Y. Kurylyak, F.Lamonaca, F.Mirabelli, "Detection of the Eye Blinks for Human's Fatigue Monitoring," in Proc. IEEE Int. Symp. Med. Meas. and Applicat. (MeMeA), Budapest, Hungary, 2012 pp. 1-4.

[78] C. C. Shih et al, "Building Long-distance Health Care Network using Minimized Portable Sensors and Active Alert System," in Proc. 16th International Conference on Network-Based Information Systems, Gwangju, Korea, 2013, pp. 401-404.

[79] B. Latre, F. Martelli, R. Rosini, C. Buratti, R. Cavallari, "A survey on wireless body area networks," Wireless Networks, vol. 17, no. 1, pp. 1-18, Jan. 2011.

[80] A. Silberschatz et al. Operating System Concepts, $9^{\text {th }}$ ed. Hoboken, NJ: Wiley, 2012.

[81] V. Joshi, P. Moradshahi, and R. Goubran. "Operating system performance measurements for Remote Patient Monitoring applications," in Proc. 2013 IEEE International Symposium on Medical Measurements and Applications (MeMeA), Gatineau, Canada, 2013, pp. 345-349.

[82] V. Joshi, M. Holtzman, A. Arcelus, R. Goubran, F. Knoefel, "Highly survivable bed pressure mat remote patient monitoring system for mHealth," in Proc. IEEE International Conference Engineering in Medicine and Biology Society (EMBS), San Diego, USA, 2012, pp. 268-271. 
[83] R. Chu, L. Gu, Y. Liu, M. Li and X. Lu, "SenSmart: Adaptive Stack Management for Multitasking Sensor Networks." IEEE Transactions on Computers, vol. 62, no. 1, pp. 137-150, Jan. 2013.

[84] D. Liming, Z. Xianchao, Q. Chenkun, G. Feng, "A real-time walking robot control system based on Linux RTAI." in Proc. International Conference on Advanced Mechatronic Systems, Luoyang, China, 2013, pp. 530-534.

[85] M. P. Hoevenaar-Blom, A. M. W. Spijkerman, D. Kromhout, J. F. van den Berg, M. Verschuren, "Sleep duration and sleep quality in relation to 12year cardiovascular disease incidence: the MORGEN study," Sleep, vol. 34, no. 11, pp. 1487, Nov. 2011.

[86] T. Kita, E. Yoshioka, H. Satoh, M Kawaharada, E. Okada, R. Kishi, "Short sleep duration and poor sleep quality increase the risk of diabetes in Japanese workers with no family history of diabetes," Diabetes care, vol. 35, no. 2, pp. 313-318, Feb. 2012.

[87] S. Stenholm, E. Kronholm, S. Bandinelli, J. M. Guralnik, L. Ferruci, "Selfreported sleep duration and time in bed as predictors of physical function decline: results from the InCHIANTI study," Sleep, vol. 34, no. 11, pp. 1583, Nov. 2011.

[88] T. M. Gill, Z. Guo, H. G. Allore, "The epidemiology of bathing disability in older persons," J. Am. Geriatr. Soc., vol. 54, no. 10, pp. 1524-1530, Oct. 2006.

[89] S. Auer, B Reisberg, "The GDS/FAST staging system," Int. Psychogeriatr, vol. 9, no. 1, pp. 167-171, Sep. 1997.

[90] S. R. Hardin, J. Dienemann, P. Rudisill, K. K. Mills, "Inpatient fall prevention: use of in-room Webcams," J. Patient. Saf., vol. 9, no. 1, pp. 2935, Mar. 2013.

[91] A. Arcelus, I. Veledar, R. Goubran, F. Knoefel, H. Sveistrup, M. Bilodeau, "Measurements of sit-to-stand timing and symmetry from bed pressure sensors," IEEE Transactions on Instrumentation and Measurement, vol. 60, no. 5, pp. 1732-1740, Apr. 2011.

[92] S. Bennett, R. Goubran, K. Rockwood, F. Knoefel, “Automated assessment of mobility in bedridden patients," 35th Annual International Conference of 
the IEEE Engineering in Medicine and Biology Society (EMBC), 2013, pp. 4271-4724.

[93] D. Townsend, R. Goubran, F. Knoefel, J. Leech, "Validation of Unobtrusive Pressure Sensor Array for Central Sleep Apnea Screening," IEEE

Transactions on Instrumentation and Measurement, vol. 61, no. 7, pp. 18571865, Jul. 2012.

[94] R. Wallace, R. Goubran, F. Knoefel, S. Marshall, M. Porter, "Measuring Variation in Driving Habits Between Drivers," in Proc. 2014 IEEE International Symposium on Medical Measurements and Applications (MeMeA), 2014, pp. 29-34.

[95] M. Rockwood, V. Joshi, K. Sullivan, R. Goubran, "Using a Real-Time Operating System for Multitasking in Remote Patient Monitoring," in Proc. 2014 IEEE International Symposium on Medical Measurements and Applications (MeMeA), 2014, pp. 475-479.

[96] Texas Instruments, "Sitara AM335x ARM Cortex-A8 Microprocessors (MPUs)," SPRS717F datasheet, Oct. 2011, [Revised April 2013].

[97] QNX, (2014), QNX Neutrino RTOS, [Online]. Available: http://www.qnX.com/products/neutrino-rtos/neutrino-rtos.html

[98] C. Silva, J. Pontes, J. Viana, L. Rocha, J. Gaspar, "A fully integrated threeaxis thermal accelerometer," in Proc. Instrumentation and Measurement Technology Conference (I2MTC), 2013 IEEE International, 2013, pp. 963966.

[99] V. Di Lecce, M. Calabrese, "Experimental system to support real-time driving pattern recognition," Advanced Intelligent Computing Theories and Applications, vol. 5227, no. 1, pp. 1192-1199, 2008. 
Appendix A:

\section{Sequence Diagram of the RPM System}




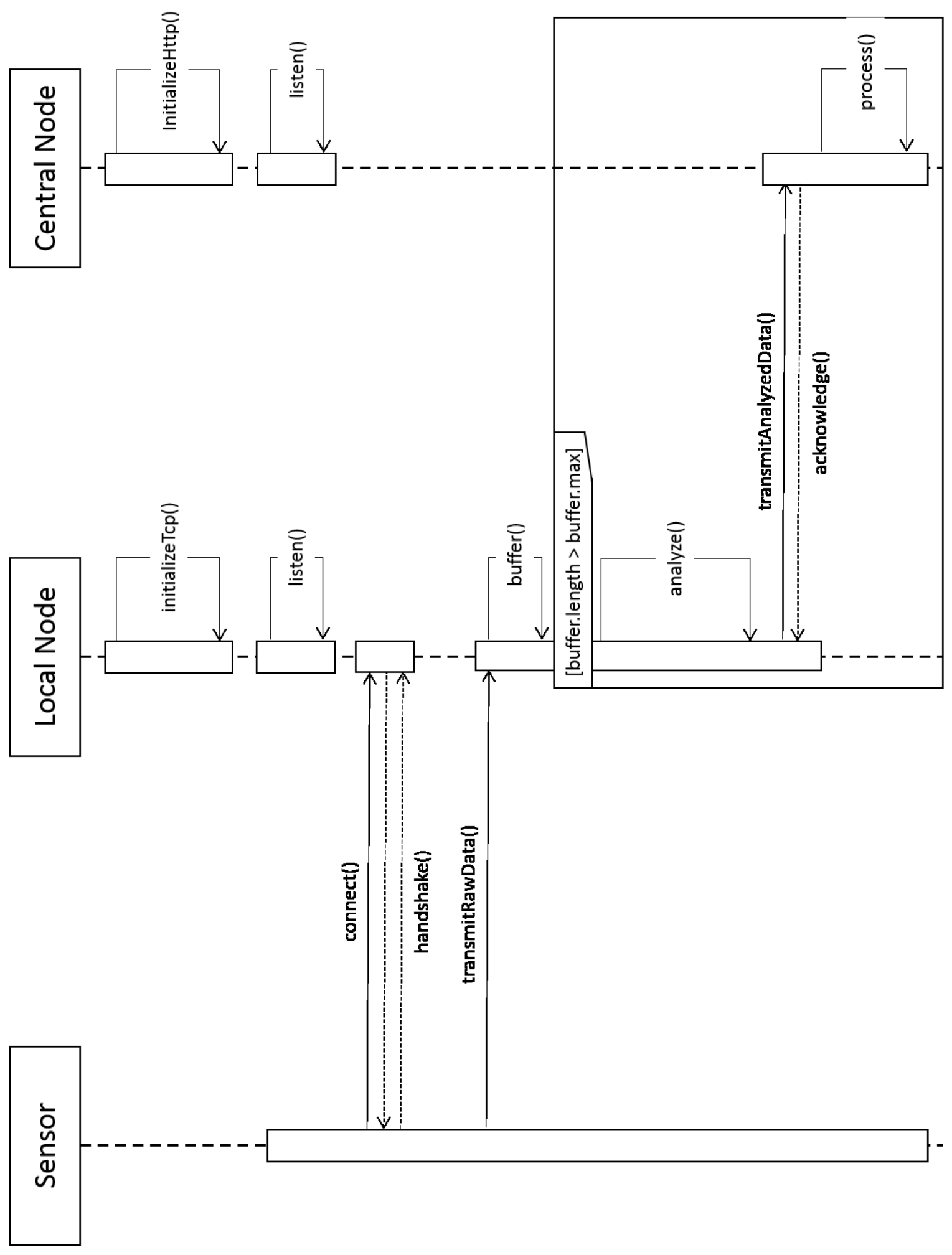

